\title{
Korngrenzsegregation in \\ Silber-Nickel und Kupfer-Wismut Legierungen
}

\author{
Dissertation \\ zur Erlangung des Doktorgrades \\ der Mathematisch-Naturwissenschaftlichen Fakultäten \\ der Georg-August-Universität Göttingen
}

vorgelegt von

Daniel Wolde-Giorgis

aus Mainz 
D7

Referent:

Prof. Dr. Kirchheim

Korreferent:

Prof. Dr. Hofsäß

Tag der mündlichen Prüfung: 25.08.2005 
„One thousandth part of antimony converts first rate best selected copper into the worst conceivable. "

Sir William Chandler Roberts-Austen (1843-1902), britischer Metallphysiker 

EINLEITUNG ............................................................................1

1 MATERIALPHYSIKALISCHER HINTERGRUND ................................. 3

1.1 GRUNDLAGEN DER KORNGRENZSEGREGATION .......................................... 3

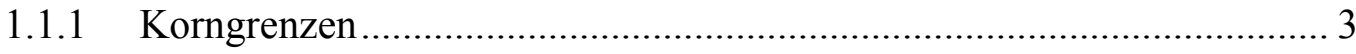

1.1.2 Thermodynamik der Segregation ........................................................ 5

1.2 LEGIERUNGSSYSTEME MIT POSITIVER MisCHUNGSENTHALPIE.................... 10

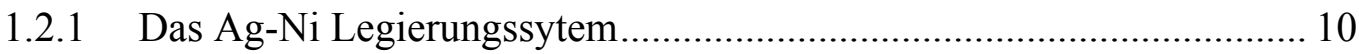

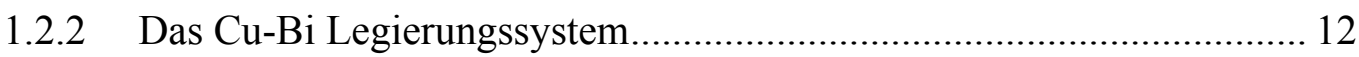

2 EXPERIMENTELLE MeTHOden................................................17

2.1 FELDIONENMIKROSKOP UND TOMOGRAPHISCHE ATOMSONDE .................... 17

2.1.1 Das Feldionenmikroskop (FIM) ..................................................... 17

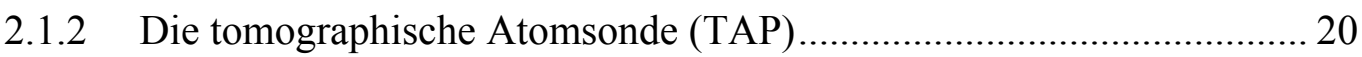

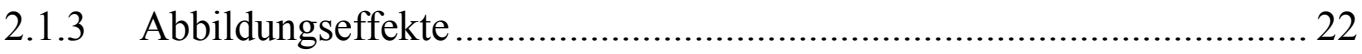

2.1.4 Auswertungsmethoden der Atomsondendaten..................................... 23

2.1.5 Parameter für FIM- und TAP-Analysen ............................................ 25

2.2 WEITERE ANALYSEMETHODEN .......................................................... 26

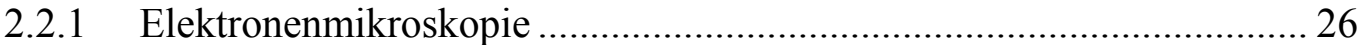

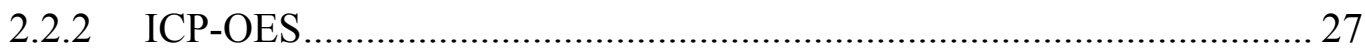

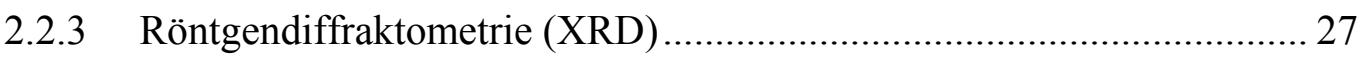

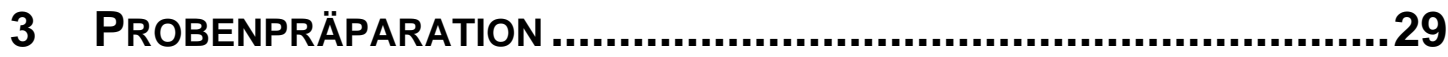

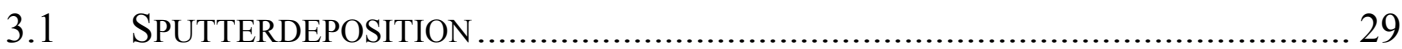

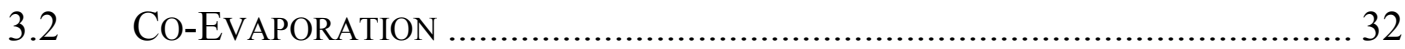

3.3 PRÄPARATION BI DOTIERTER $\Sigma$ 19A BIKRISTALLE ...................................... 34

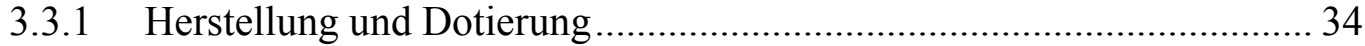

3.3.2 Vorgehensweisen zur Spitzenpräparation ........................................... 36

3.4 SiMULATION DER FELDVERTEILUNG AN FIM/TAP SPITZEN ........................ 40

3.5 ZIELPRÄPARATION WOHLDEFINIERTER KORNGRENZEN .............................. 44

4 ERGEBNISSE ...............................................................47

4.1 UNTERSUCHUNG DER CO-EVAPORIERTEN AG-Ni PROBEN............................ 47

4.1.1 Nanostruktur des ,as-prepared“-Zustandes.......................................... 47

4.1.2 Ni-Verteilung in wärmebehandelten Proben......................................... 56

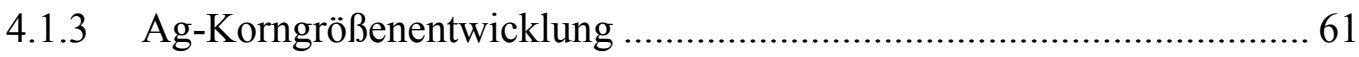


4.2 UNTERSUCHUNG DER Ag-Ni MULTILAGEN............................................... 64

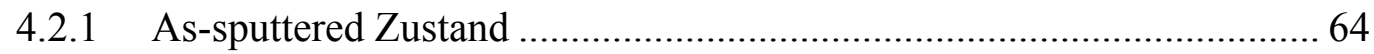

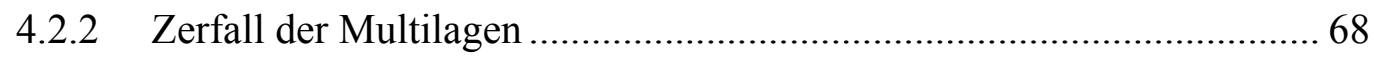

4.3 UNTERSUCHUNG DER SPUTTER-DEPONIERTEN CU-BI-LEGIERUNG................ 71

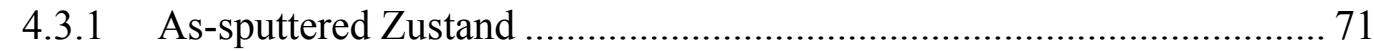

4.3.2 Exzess an Kleinwinkelkorngrenzen ............................................... 74

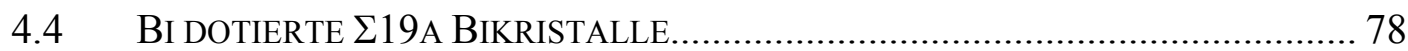

4.4.1 Verifizierung der $\Sigma 19 a$ Korngrenze ................................................... 78

4.4.2 Bi-Versprödung der Korngrenze ...................................................... 79

4.4.3 Bestimmung des Korngrenzenexzesses ........................................... 79

4.4.4 Facettierung an der Korngrenze ...................................................... 82

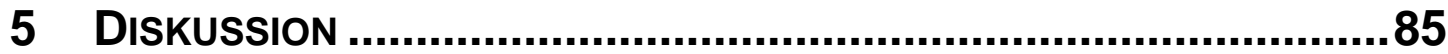

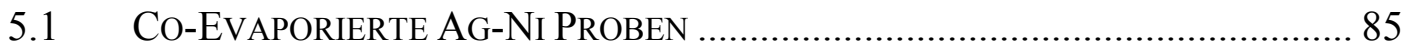

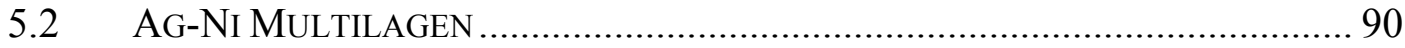

5.2.1 Modell des Schichtzerfalls ................................................................. 90

5.2.2 Mittlerer Oberflächendiffusionskoeffizient ........................................ 93

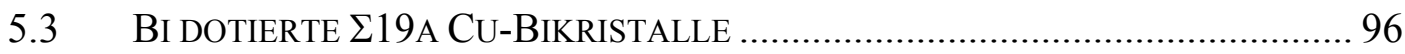

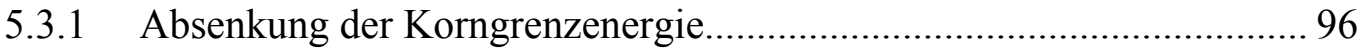

5.3.2 Segregation an Kleinwinkel-Korngrenzen .......................................... 99

5.3.3 Lunkerstabilisierung durch Oberflächensegregation ......................... 100

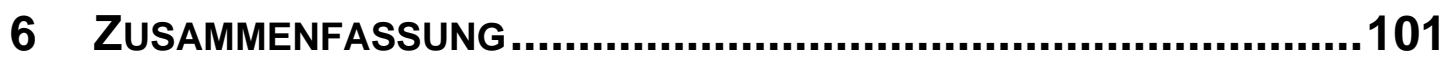

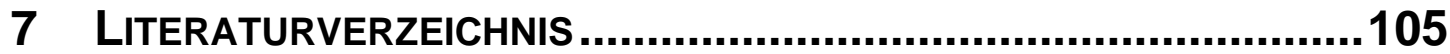

8 APPENDIX: SimULATIONEN ZUR SPUTtER-DEPOSITION .............. 109 


\section{Einleitung}

Die Eigenschaften moderner Materialien werden stark durch die Beschaffenheit ihrer Nanostruktur kontrolliert. Materialien mit Korngrößen unterhalb $100 \mathrm{~nm}$ weisen oft viele technologisch interessante Eigenschaften auf, wie besondere Härte, Abriebbeständigkeit und Korrosionsresistenz. Die ständige Miniaturisierung elektronischer Bauteile in den letzten Dekaden, wie auch das Verständnis der makroskopischen Materialeigenschaften, erfordern daher eine genaue Kenntnis der Prozesse an den Korngrenzen auf atomarer Skala. Anwendungen zur Steigerung der Datendichte magnetischer Aufzeichnungssysteme ergeben sich beispielsweise durch die isolierte Einbettung ferromagnetischer Eindomänenkörner in eine nichtferromagnetische Matrix. Daraus ergibt sich eine hohe Korngrenzdichte. Die damit verbundene hohe freie Energie solcher Materialien kontrolliert zu einem hohen Maß die ablaufenden Prozesse bei thermischen Behandlungen. Die Temperatur, bei der üblicherweise die Rekristallisation einsetzt, ist bei diesen Materialen deutlich geringer als im polykristallinen Material. Die Veränderung der Nanostruktur verursacht in solchen Fällen einen schnellen Verlust der technologisch attraktiven Eigenschaften.

Interessanterweise zeigen einige nanokristalline Materialien nach einer thermischen Behandlung eine erhebliche Versprödung, die oft auf die bevorzugte Segregation einer Legierungskomponente an den Korngrenzen zurückgeführt werden kann. In anderen Legierungssystemen trägt eine solche Segregation wiederum deutlich zur thermischen Stabilität des nanokristallinen Gefüges bei. Die Exzess-Atome sättigen die freien Bindungen am Ort der Korngrenzen und senken somit die Korngrenzenenergie ab. Es ist vorstellbar, dass dadurch die treibende Kraft der Rekristallisation minimiert wird oder vollständig verschwinden kann.

Im Rahmen dieser Arbeit wird diese Fragestellung für die zwei stark entmischenden Legierungssysteme $\mathrm{Ag}-\mathrm{Ni}$ und $\mathrm{Cu}-\mathrm{Bi}$ behandelt. Ersteres ist aufgrund seiner Eigenschaften interessant für technologische Anwendungen (GMR-Sensoren, Speichermedien), während Letzterem, aufgrund der starken Versprödung an den Korngrenzen, eine wichtige grundlagenphysikalische Relevanz zukommt. Beide Legierungssysteme weisen im thermodynamischen Gleichgewicht praktisch keine Löslichkeit auf und haben deutlich unterschiedliche Atomradienverhältnisse von $\mathrm{Ni} / \mathrm{Ag}=15,6 \%$ und $\mathrm{Cu} / \mathrm{Bi}=30,8 \%$. Die Stapelfehlerenergien von $\mathrm{Ag}$ und $\mathrm{Cu}$ sind 
mit $20 \mathrm{~mJ} / \mathrm{m}^{2}$ bzw. $40 \mathrm{~mJ} / \mathrm{m}^{2}$ klein. Beide Systeme sollten daher vergleichbare Korngrenzenergien aufweisen. Zum Verständnis der ablaufenden Prozesse an den Korngrenzen werden in dieser Arbeit Proben verschiedener Herstellungsprozesse untersucht und charakterisiert. Dies erfordert eine Untersuchungsmethode mit höchster Auflösung bei gleichzeitiger chemischer Analyse.

Diese Arbeit gliedert sich in sechs Kapitel. Im ersten Kapitel werden die untersuchten Legierungssysteme vorgestellt und es wird eine Übersicht über die Grundlagen der Korngrenzsegregation gegeben. Im zweiten Kapitel werden die experimentellen Methoden erläutert. Der Schwerpunkt liegt dabei auf der Tomographischen Atomsonde (TAP) und den verwendeten Auswerteverfahren. Das TAP ist mit einer räumlichen Auflösung auf der Subnanometerskala und einer chemischen Auflösung bis zu wenigen 100 ppm bestens für die Untersuchung der Korngrensegregation geeignet. Zusätzlich können mit Hilfe der Feld-Ionen-Mikroskopie ergänzende Erkenntnisse über die Korngröße des Materials gewonnen werden. Der Einsatz dieser Methoden erfordert eine besondere, spitzenförmige Probengeometrie. Für die gezielte Untersuchung einzelner Korngrenzen wurde daher ein neues Verfahren der Probenpräparation entwickelt. Dieses wird, wie alle anderen Arbeitsschritte zur Probenpräparation, im dritten Kapitel vorgestellt. Im vierten Kapitel werden die Ergebnisse der dreidimensionalen Atomsondenanalysen und feldionenmikroskopischen Untersuchungen aufgeführt. Die Kombination beider Methoden wird dabei an vielen Stellen ausgenutzt, da die Interpretierbarkeit der TAP-Ergebnisse oft durch die kleinen lateralen Abmessungen des Analysevolumens erschwert ist. Die Proben der beiden Legierungssysteme Ag-Ni und $\mathrm{Cu}-\mathrm{Bi}$ wurden jeweils auf zwei verschiedene Arten hergestellt. Beide Legierungen wurden als dünne Schichten mittels Sputterdeposition präpariert. Zusätzlich wurden nanokristalline Ag-Ni Gefüge bzw. Bi-dotierte $\mathrm{Cu}$-Bikristalle untersucht. Dies ermöglicht es innerhalb eines Legierungssystems, unterschiedliche Reaktionsabläufe verschiedener Probenarten $\mathrm{zu}$ vergleichen und diese auf die entsprechenden Mikrostrukturen und ihren Herstellungsprozess zurückzuführen. Im fünften Kapitel findet sich ein Überblick über die ermittelten Nanostrukturen. Diese werden in Abhängigkeit ihres Herstellungsprozesses diskutiert. Die Ergebnisse werden im letzten Kapitel zusammengefasst. 


\section{Materialphysikalischer Hintergrund}

\subsection{Grundlagen der Korngrenzsegregation}

\subsubsection{Korngrenzen}

Korngrenzen $(\mathrm{KG})$ sind atomar scharfe Übergangsbereiche zwischen unterschiedlich orientierten Bereichen eines Kristalls. Sie stellen, abhängig von ihrer Fehlpassung, eine mitunter erhebliche Störung des Kristallaufbaus dar. Die dabei gespeicherte Energie wird über die spezifische Korngrenzenergie $\gamma$ als Differenz $G^{\prime}$ der freien Energien desselben Kristalls eines reinen Materials mit und ohne KG dividiert durch die KGFläche definiert.

$$
\gamma=\left(\frac{\partial G^{\prime}}{\partial A_{K G}}\right)_{T, P, n}
$$

Die KG-Energie ist somit ein Maß für den Energieaufwand, der benötigt wird um weitere KG-Fläche zu schaffen.

Eine Korngrenze kann makroskopisch durch die Verdrehung zweier Kristallite gegeneinander, d.h. Drehachse und Winkel, und durch die Orientierung der Grenze selbst beschrieben werden. Insgesamt werden also fünf makroskopische Variablen benötigt. Auf mikroskopischer Ebene treten noch weitere Freiheitsgerade auf, wie z.B. leichte Translationen der angrenzenden Kristallite und Relaxationen der atomaren Positionen. Setzt man in Gedanken die Atompositionen über die Korngrenze in den jeweils anderen Kristall fort, so stellt man für spezielle Orientierungen der Kristallite das Auftreten von Koinzidenzpunkten fest. Diese spannen, aufgrund der periodischen Anordnung der Atome im Kristall, ebenfalls ein Gitter auf. Der inverse Bruchteil der Atome auf Koinzidenzplätzen wird als $\Sigma$-Wert bezeichnet. Durch diesen Wert wird die Lage der Korngrenze im Kristall jedoch nicht beschrieben. Oft beschränkt man sich daher bei der Erzeugung von Bikristallen auf eine kohärente Zwillingslage der Korngrenze. Diese nimmt zu beiden Kristallhälften denselben Kippwinkel ein und kann 
damit makroskopisch durch die Angabe des Ebenentyps vollständig beschrieben werden. Je kleiner $\Sigma$ ist, desto besser geordnet ist die Korngrenze. So können Kleinwinkelkorngrenzen $^{1}$ mit $\Sigma=1$ beschrieben werden, da nahezu alle Atompositionen bis auf die Versetzungskerne Koinzidenzgitterpunkte sind. Dementsprechend wird für höhere $\Sigma$-Werte mit größeren Fehlpassungen an der Korngrenze eine wesentlich höhere Korngrenzenergie erwartet. In dieser Arbeit wurde die Bi-Segregation an einer symmetrischen $\Sigma 19 a\{331\}$ Korngrenze eines Cu-Bikristalls untersucht. Diese wird konstruiert indem zwei um einen Winkel von $26,51^{\circ}$ zueinander verdrehte $<110>$-orientierte Einkristalle so zusammengefügt werden, dass die Lage der Kontaktfläche kohärent ist. Das zugehörige Koinzidenzgitter ergibt sich, indem die Gitter beider Kristallhälften ineinander fortgesetzt werden, wobei auffällt, dass jede 19 . $\{331\}$ - bzw. alternativ jede $\{116\}$-Atomlage eine Koinzidenzlage darstellt (siehe Abb. 1.1). Einige $\Sigma$-Werte können auf verschiedenen Wegen eingestellt werden. Geometrisch kann $\Sigma=19$ auch durch eine Drehung beider Einkristalle um die $<111>$-Achse um einen beidseitigen Winkel von 46,8 erfolgen. Zur Unterscheidung der Fälle bezeichnet man diese Konfiguration daher als $\Sigma 19 b$ Korngrenze.

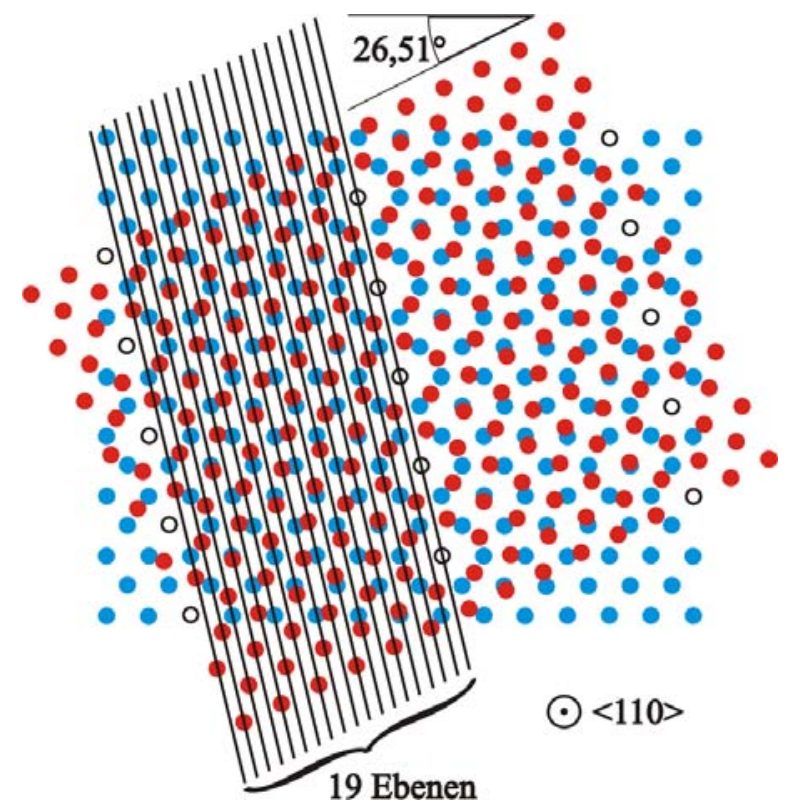

Abb. 1.1: $\Sigma 19$ a Korngrenze und Koinzidenzgitter; offene Symbole sind Koinzidenzatome

\footnotetext{
${ }^{1}$ Als Kleinwinkelkorngrenzen bezeichnet man solche mit Kipp- oder Drehwinkeln kleiner als $15^{\circ}$.
} 


\subsubsection{Thermodynamik der Segregation}

In einer binären Legierung $\mathrm{AB}$ können B-Atome bevorzugt zu den Korngrenzen der AMatrix diffundieren und dort flächige Anreicherungen bilden (Abb. 1.2). Man bezeichnet dies dann als B-Segregation. Die freie Enthalpie nimmt bei konstantem Druck $\mathrm{p}$ und konstanter Temperatur T ein Minimum an. Sie ist daher zur Beschreibung der untersuchten Prozesse geeignet.

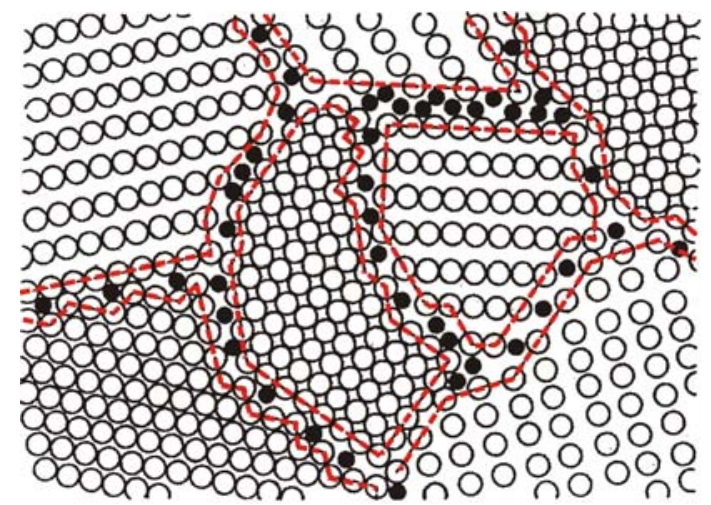

Abb. 1.2: Schematische Darstellung der Segregation; A-Atome hell, B-Atome dunkel

Im Fall eines Bikristalls, bestehend aus zwei Hälften verschiedener Kristallorientierungen $I$ und $I I$ und einer eingeschlossenen Korngrenze der Fläche $A_{K G}$ (Abb. 1.3), berechnet sich die zugehörige freie Enthalpie $G^{\prime}$ ganz allgemein über

$$
G^{\prime}=U+p V-T S
$$

Aufgrund von nicht vollständig abgesättigten atomaren Bindungen über die Korngrenze hinweg kann eine freie Grenzflächenenthalpie $G_{G F}$ der Dimension Energie/Fläche als Differenz zwischen $G$ ' und der Summe derjenigen Energiebeiträge definiert werden, die zum Aufbau beider Kristallhälften des Bikristalls nötig sind. 


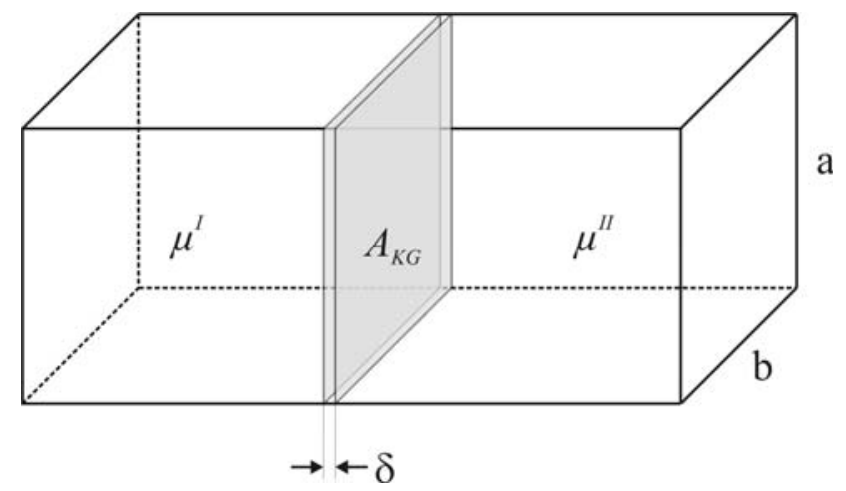

Abb. 1.3: Schematischer Aufbau des betrachteten Bikristalls

Der Korngrenze wird eine Dicke $\delta$ zugeordnet, die senkrecht zur Korngrenze gemessen die Ausdehnung des kristallographisch gestörten Bereichs angibt. Die Korngrenzdicke wird typischerweise mit $\delta=5 \cdot 10^{-10} \mathrm{~m}$ angegeben [HEUMANN92], womit sich eindeutig zwei Kristallhälften definieren lassen. Für die freie Grenzflächenenthalpie folgt

$$
G_{G F} A_{K G}=G^{\prime}-\left(\sum_{i} \mu_{i}^{I} n_{i}^{I}+\sum_{i} \mu_{i}^{I I} n_{i}^{I I}\right)
$$

Die spezifische Korngrenzenergie $\gamma_{K G}$ ist daher als Änderung der freien Enthalpie G' des Kristalls definiert

$$
\gamma_{K G}=\left(\frac{\partial G^{\prime}}{\partial A_{K G}}\right)_{T, p, n}
$$

Für das totale Differential von $G$ ' folgt durch Legendre-Transformation

$$
d G^{\prime}=-S d T+V d p+\sum_{i} \mu_{i} d n_{i}+\gamma_{K G} d A_{K G}
$$


Eine Vergrößerung der Korngrenzfläche um $d A_{K G}$ bei $T, p=c o n s t$ führt zu einer Abnahme der Atomanzahlen in den jeweiligen Körnern um $d n_{i}{ }^{I}$ bzw. $d n_{i}{ }^{I I}$. Ein Vergleich von (1.3) und (1.5) ergibt somit

$$
G_{G F} d A_{K G}=\gamma_{K G} d A_{K G}+\left(\sum_{i} \mu_{i}^{I} d n_{i}^{I}+\sum_{i} \mu_{i}^{I I} d n_{i}^{I I}\right)
$$

Da für den betrachteten Bikristall kein Teilchenaustausch mit anderen Systemen möglich ist, gilt die Massenerhaltung. Es wird ferner angenommen, dass mobile Atome einer Minoritätskomponente, oder Verunreinigungen am Ort der Korngrenze segregieren können, um dort die freien Bindungen $\mathrm{zu}$ sättigen. Um solche flächigen Anreicherungen zu berücksichtigen wird der Korngrenzexzess $\Gamma_{K G}$ mit der Dimension Atome/Fläche eingeführt. Da ein Teilchenaustausch zwischen den beiden Kristallhälften möglich ist, gilt

$$
d n_{i}^{I}+d n_{i}^{I I}=-\Gamma_{i} d A_{K G}
$$

Im thermodynamischen Gleichgewicht müssen die chemischen Potentiale beider Hälften des Bikristalls gleich sein, d.h. $\mu_{i}^{I}=\mu_{i}^{I I}=\mu_{i}$. Damit kann (1.6) geschrieben werden als

$$
\begin{aligned}
& G_{G F} d A_{K G}=\gamma_{K G} d A_{K G}+\sum_{i} \mu_{i} \Gamma_{i} d A_{K G} \\
& \Leftrightarrow \quad G_{G F}=\gamma_{K G}+\sum_{i} \mu_{i} \Gamma_{i}
\end{aligned}
$$

Die freie Grenzflächenenthalpie $G_{G F}$ kann somit als Summe der spezifischen Korngrenzenergie $\gamma_{K G}$ und der Beiträge der freien Energie der ungesättigten Bindungen der Exzessatome ausgedrückt werden. Für das totale Differential $d G_{G F}$ folgt dann

$$
d G_{G F}=d \gamma_{K G}+\sum_{i} \mu_{i} d \Gamma_{i}+\sum_{i} \Gamma_{i} d \mu_{i}
$$


Betrachtet man den Fall $A_{K G}, T, p=$ const, so kann eine Änderung der freien Enthalpie $G$ ' des Bikristalls nur durch die Änderung der freien Grenzflächenenthalpie verursacht werden. Es gilt daher $d G_{G F}=d G^{\prime} / A_{K G}$. Mit (1.5) und $d \Gamma=d n_{i} / A_{K G}$ folgt

$$
d G_{G F}=\sum_{i} \mu_{i} d \Gamma_{i}
$$

Der Vergleich von (1.10) und (1.11) ergibt

$$
d \gamma_{K G}=-\sum_{i} \Gamma_{i} d \mu_{i}
$$

In einem binären Legierungssystem A-B kann so die Gibbs'sche Adsorptionsisotherme für $\Gamma_{A}=0$ am Ort der Korngrenze abgeleitet werden

$$
\Gamma_{B}=-\left(\frac{d \gamma_{K G}}{d \mu_{B}}\right)=-\frac{1}{R T}\left(\frac{d \gamma_{K G}}{d \ln a_{B}}\right)
$$

Für ein verdünntes binäres Legierungssystem kann eine nützliche Abwandlung der Gibbs'schen Adsorptionsisotherme angegeben werden. Besteht der Bikristall aus der Majoritätskomponente A und der Minoritätskomponente B in stark verdünnter molarer Lösung $c_{B}<<1$, so kann die Aktivität der B-Atome nach Henrys Gesetz (Abb. 1.4) ausgedrückt werden als $a_{B}=$ const $\cdot c_{B}$, woraus folgt

$$
\left(\frac{d \gamma_{K G}}{d \ln c_{B}}\right)=-R T \Gamma_{B}
$$

Diese Gleichung kann experimentell eingesetzt werden, um aus der Messung des Korngrenzexzesses $\Gamma_{B}$ nach einer Segregationsbehandlung bei fester Temperatur $T$ und bei bekannter homogener Dotierung $c_{B}$ die Absenkung der Korngrenzenergie $\gamma_{K G} \mathrm{zu}$ bestimmen. 


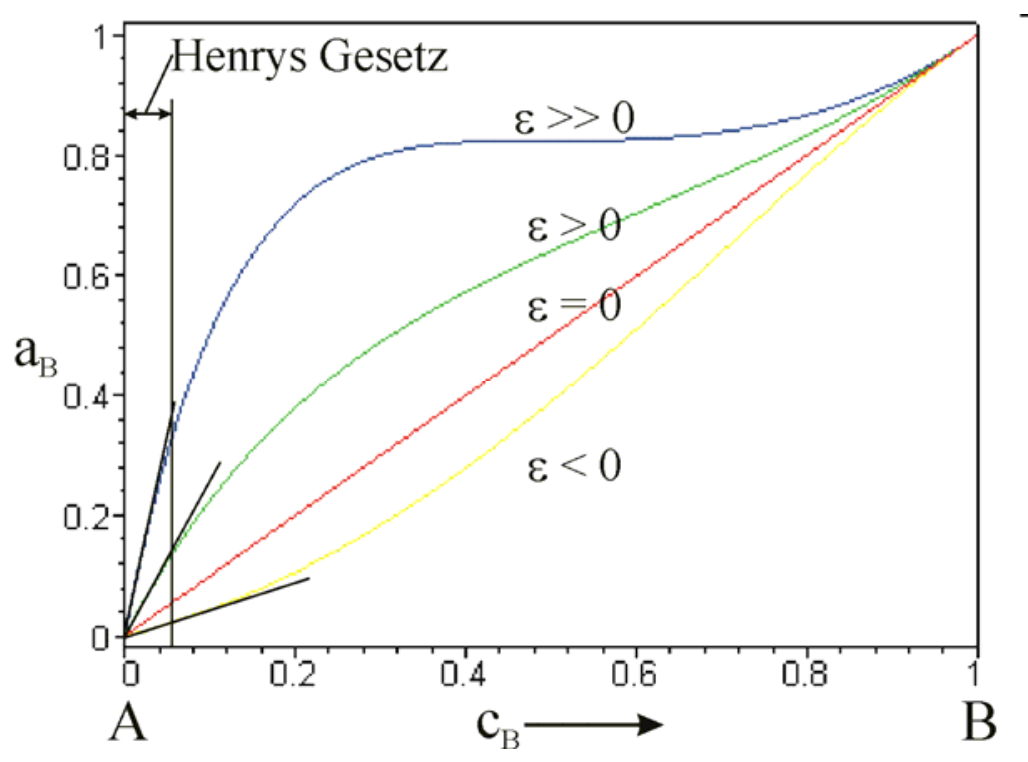

Abb. 1.4: Gesetz von Henry als Grenzfall für den Verlauf der Aktivität der B-Atome in Abhängigkeit der Konzentration für verschiedene Paarvertauschungsparameter $\varepsilon$

Eine notwendige Voraussetzung für die Gültigkeit von Gleichung 1.14 ist also eine geringe Dotierung der A-Matrix mit einer Atomsorte B. Die treibende Kraft zur Segregation kann durch die Wahl eines unmischbaren Legierungssystems vergrößert werden. Hierbei ist jedoch zu beachten, dass Segregations- und Ausscheidungsvorgänge konkurrierend ablaufen können. Es zeigt sich, dass Atome mit einem größerem Atomradius oft bevorzugt in die Korngrenzen der Matrix mit kleineren Atomen segregieren. Kupfer bietet sich mit einer geringen Löslichkeit von Wismut bis 200 ppm und einem deutlichen Unterschied der Atomgrößen als geeignetes Modellsystem an. Es existiert schon im mischbaren Bereich eine hohe treibende Kraft zur Segregation, weswegen Ausscheidungsvorgänge nicht berücksichtigt werden müssen. Auch das Silber-Nickel-Legierungssystem weist einen deutlichen Unterschied in den Atomgrößen auf. Durch die deutliche Mischungslücke im festen wie auch im flüssigen Zustand bietet sich dieses System ebenfalls für eine weitere Untersuchung der Korngrenzsegregation an. 


\subsection{Legierungssysteme mit positiver Mischungsenthalpie}

Die Entwicklung und das Verständnis neuer Legierungen gehören zu den wichtigsten Themenbereichen der modernen Materialphysik. In den letzten Jahrzehnten wurden durch neue Prozesstechniken verstärkt Legierungen hergestellt, deren Elemente im thermodynamischen Gleichgewicht ineinander unlöslich sind. Neue Analysemethoden eröffnen neuerdings auch eine direkte Darstellung der Nanostrukturen solcher Legierungen und deren Entmischungsreaktionen bei thermischen Behandlungen. In den folgenden Abschnitten werden die zwei untersuchten binären Legierungssysteme dieser Kategorie vorgestellt.

\subsubsection{Das Ag-Ni Legierungssytem}

Das Ag-Ni-System (Abb. 1.5) zeichnet sich durch seine geringe Löslichkeit der Konstituenten in der festen und einer partiellen Löslichkeit in der flüssigen Phase aus. Dies lässt sich durch eine stark positive Mischungsenthalpie erklären. Diese ist mit $\Delta H=+23 \mathrm{~kJ} / \mathrm{mol}$ so hoch, dass auch die Zunahme der Konfigurationsentropie im flüssigen Zustand keinen ausgedehnten Mischungsbereich ermöglicht. Bei dem vorliegenden Atomgrößenverhältnis von $\mathrm{Ag} / \mathrm{Ni}=15,6 \%$ wird bereits die erste HumeRotherysche Regel der Stabilität einer Mischphase verletzt. Es sind bei Atomgrößenverhältnissen $>15 \%$ auch amorphe Phasen vorstellbar, die durch Untersuchungen von Ma [MA05] im Bereich hoher Ni-Übersättigungen experimentell nachgewiesen werden konnten. Die Gitterfehlpassung der Ag- und Ni-Gitter beträgt in $<100>$-Richtung 16,2\%. Somit sind auch elastische Verzerrungen der Grenzflächen bei der Herstellung von technisch relevanten Schichtensystemen zu beachten. Unter Zugabe von 20 At.\% Eisen können $\mathrm{Ag} / \mathrm{Ni}_{80} \mathrm{Fe}_{20}\left(\mathrm{Ni}_{80} \mathrm{Fe}_{20}\right.$ : Permalloy, Py) Multilagen durch Sputterdeposition oder gepulste Laserdeposition (PLD) hergestellt werden, die schon in kleinen magnetischen Feldern einen GMR (Giant Magneto Resistance) Effekt zeigen [FAUPEL02]. 


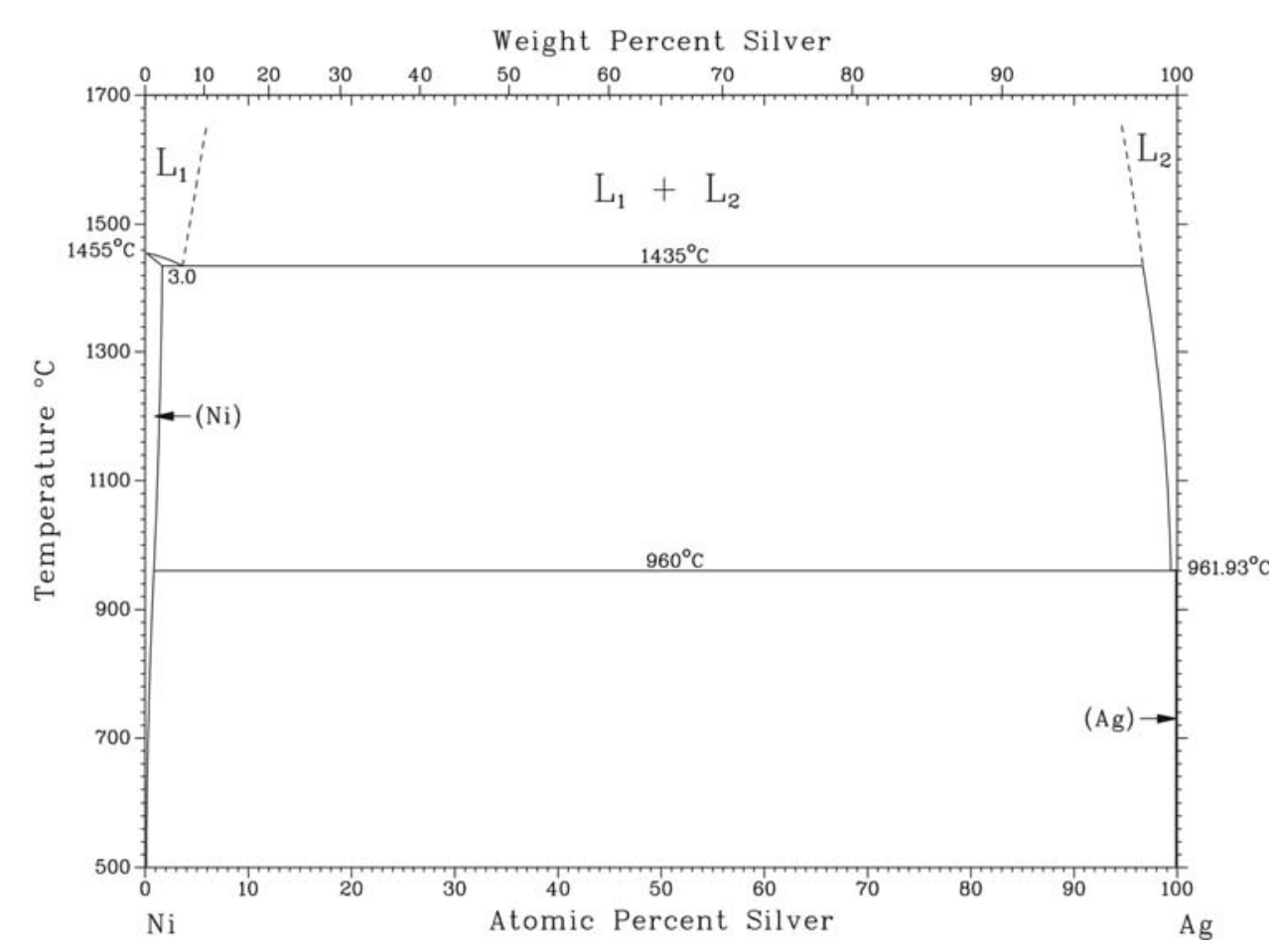

Abb. 1.5: Ag-Ni Phasendiagramm nach Massalski [MASSALSKI90]

Die erzeugten Multilagen zeigen dieses Verhalten jedoch erst nach einer thermischen Behandlung, bei der die Anwesenheit magnetischer Py-Inseln in der Ag-Matrix den GMR-Effekt begünstigt. Die Transformation des kontinuierlichen Schichtpakets in einen diskontinuierlichen Zustand kann somit genutzt werden, um z.B. den idealen Arbeitspunkt eines GMR-Sensors einzustellen. Der zugrunde liegende Mechanismus wird oft als Ag-Brückenbildung durch die Ni-reichen Schichten identifiziert [BorChERS96], [PARKER94]. Schweitz et al. [SCHWEITZ01] untersuchten den Einfluss der Grenzflächen und der Verzerrungsenergie zwischen den Schichten im Ag-Ni System und zeigten, dass diese bei einer thermischen Behandlung bei $533 \mathrm{~K}$ für $22 \mathrm{~h}$ ebenfalls zunächst Ag-Brückenbildungen aufweisen, bevor diese in Ag- und Ni-Cluster zerfallen (Abb. 1.6). Die sputterdeponierten Schichten zeigten im Ausgangszustand eine kolumnare Kornstruktur mit einem Krongrößenverhältnis von $31 \mathrm{~nm} / 159 \mathrm{~nm}$ (inplane/out-of-plane). Diese zerfielen bei höheren Temperaturen in sphärische Körner mit einem Radius von ca. $40 \mathrm{~nm}$. 

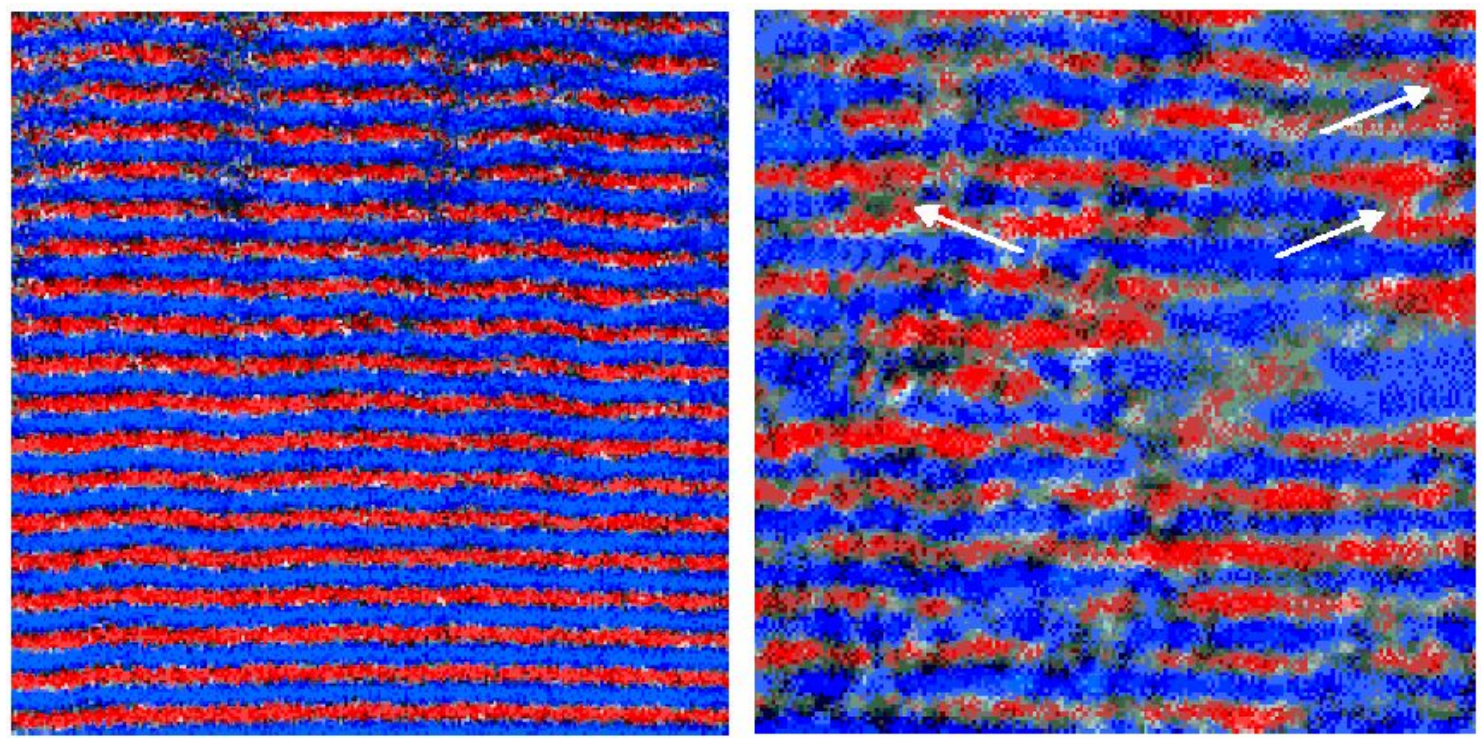

Abb. 1.6: Ag-Brückenbildung (Pfeile) in Ag(rot)/Ni(blau)-Multilagen; chemisches Verteilungsbild (EFTEM), $80 \times 80 \mathrm{~nm}^{2}$; links: as-deposited, rechts: nach thermischer Behandlung 533 K für 22 h, [SCHWEITZ01]

Der Transportmechanismus, der zur Brückenbildung führt, konnte mit den eingesetzten Methoden auf atomarer Skala jedoch nicht geklärt werden. So bleibt offen, welche Rolle Korngrenzen als mögliche Flächen bevorzugter Segregation einer Komponente, bzw. schneller Diffusionswege spielen. Auch in der vorliegenden Arbeit wurden solche Multilagen untersucht. Dabei wurden gezielt Ag-Korngrenzen untersucht und ein direkter Vergleich mit den Beobachtungen von Schweitz et al. angestrebt.

\subsubsection{Das Cu-Bi Legierungssystem}

Das Cu-Bi System ist ein bekanntes Beispiel für eine extrem versprödende Legierung. Vor über hundert Jahren wurden die ersten systematischen Untersuchungen dazu durchgeführt. 1874 berichtete Hampe [HAMPE74] von einem Übergang von duktilem, transgranularem zu sprödem, intergranularem Bruchverhalten in polykristallinem $\mathrm{Cu}$. In Analogie zum eingangs angeführten Zitat des englischen Metallphysikers Sir William Chandler Roberts-Austen zur Antimonversprödung des Kupfers sind auch im $\mathrm{Cu}-\mathrm{Bi}$ Legierungssystem nur kleinste Mengen von Bi erforderlich, um diesen Übergang zu induzieren. Voce und Hallowes [Voce47] folgerten schon 1947 aus Bruchexperimenten, dass dünne, intergranulare Bi-Filme für das Sprödbruchverhalten 
des polykristallinen $\mathrm{Cu}$ verantwortlich sind. Konsistent $\mathrm{zu}$ dieser Folgerung konnten Hondros et al. [HoNDROS74] zeigen, dass für unterschiedliche Bi-Konzentrationen in $\mathrm{Cu}$-Einkristallen keine messbare Versprödung auftritt. Diese Befunde verdeutlichen die Notwendigkeit, die Korngrenzsegregation auf atomarer Skala zu untersuchen.

Die Herausforderung bei der Untersuchung dieses Systems liegt in der Tatsache, dass sich die entscheidende Korngrenzsegregation bereits im Bereich geringster Konzentrationen (wenige $10 \mathrm{At} . \% \mathrm{ppm}$ ) ereignen. Neben der Notwendigkeit, diesen Bereich chemisch auflösen zu können, muss für die Beobachtung eine Messmethode verwendet werden, die zusätzlich eine atomare Auflösung der Korngrenze zu liefern im Stande ist. Die Randlöslichkeit von $\mathrm{Bi}$ in $\mathrm{Cu}$ wird im Phasendiagramm nach Massalski (Abb. 1.7) nur ungenügend angegeben. Neuere Untersuchungen von Chang et al. [CHANG97A] zeigen eine Löslichkeit von bis zu 200 ppm $\mathrm{Bi}$ in $\mathrm{Cu}$ (Abb. 1.8).

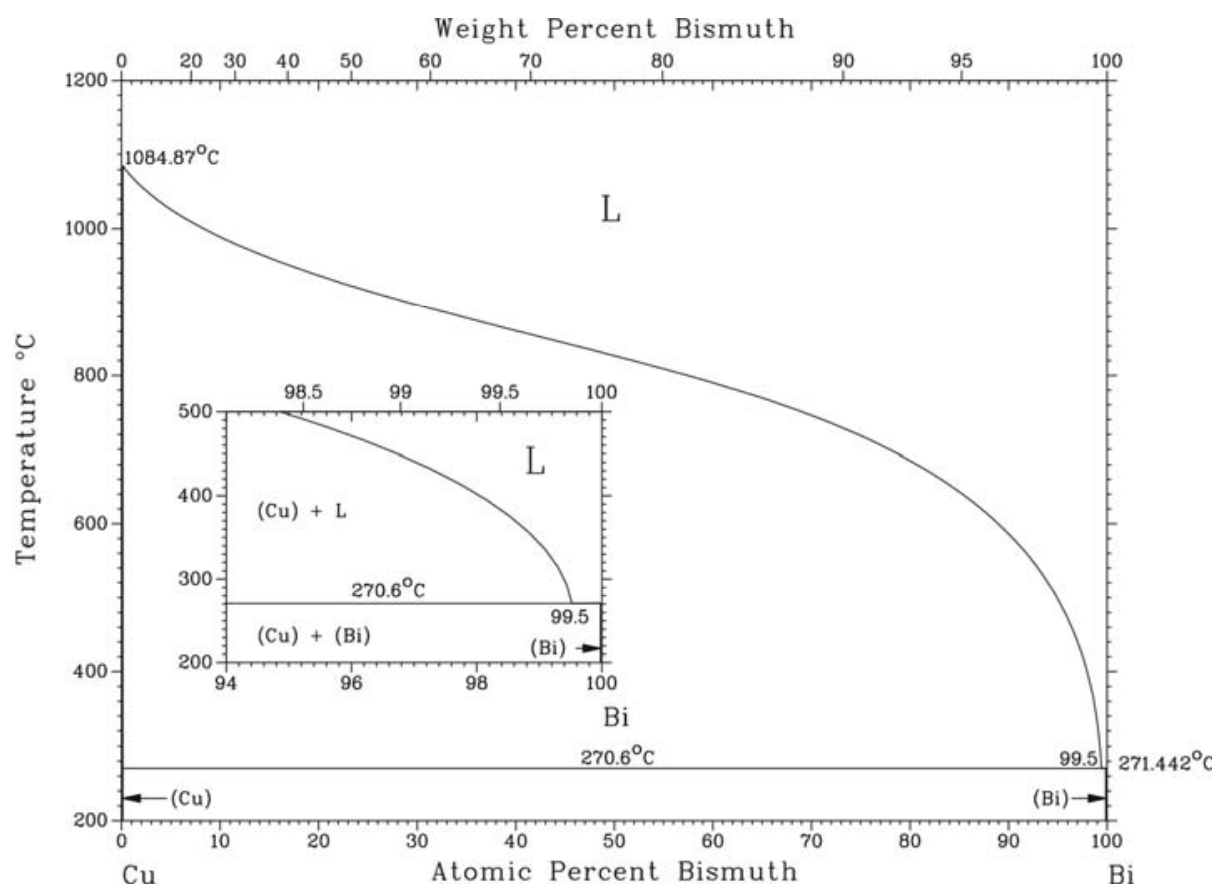

Abb. 1.7: Cu-Bi Phasendiagramm nach Massalski [MASSALSKI90]

Um die Bi-Segregation im Cu-Bi-Legierungssystem $\mathrm{zu}$ untersuchen wurden die Exzesswerte mit Hilfe der Auger-Elektronen-Spektroskopie (AES) an in-situ gebrochenen Bi-dotierten Cu-Polykristallen bestimmt [CHANG99B]. Der Bruch ist dabei ein einfaches Verfahren, um eine versprödete Korngrenze freizulegen. Ein wesentliches Problem ergibt sich aus der Tatsache, dass die Korngrenzen zunächst eine hinreichende Versprödung aufweisen müssen, um überhaupt mit dieser Methode analysiert werden zu 
können. Es zeigt sich tatsächlich, dass bevorzugt stark versprödete Korngrenzen brechen, wodurch bei der AES-Analyse der mittlere Exzess an Korngrenzen deutlich überschätzt wird.

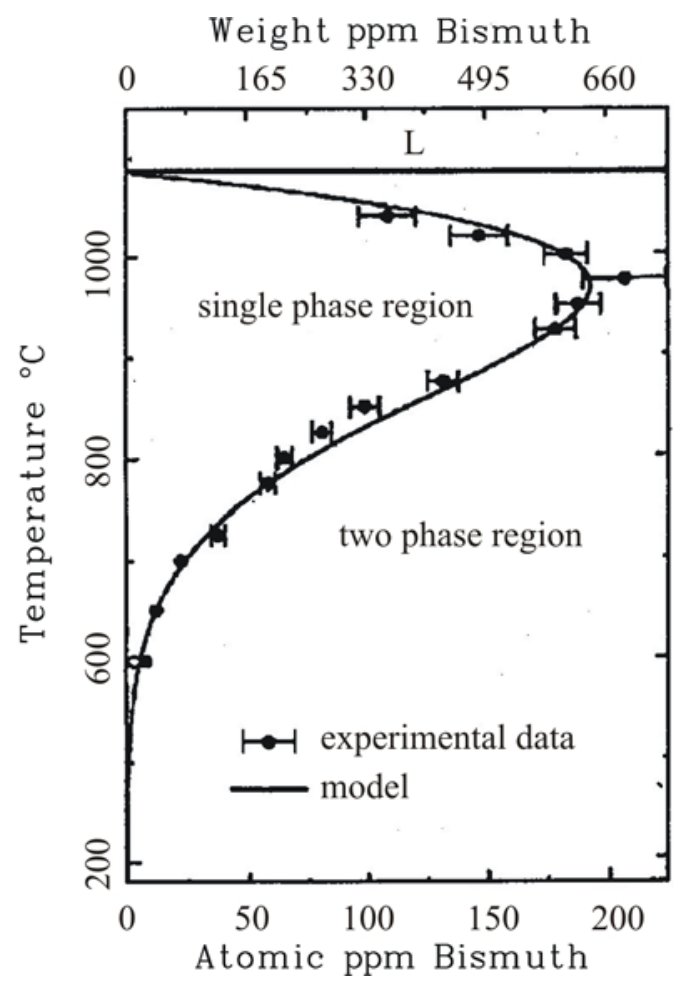

Abb. 1.8: Cu-BiPhasendiagramm bei geringen Bi-Gehalten nach Chang et al. [Chang97a]

Bei einer Temperatur von $850^{\circ} \mathrm{C}$ beobachten Schölhammer et al. [SCHÖLHAM01] eine Segregation von $\mathrm{Bi}$ in $\mathrm{Cu}-$ Korngrenzen. Sie schließen über Raster-Kraftmikroskopische Messungen der Korngrenz-Höhenprofile am Durchstoßpunkt einer $\Sigma 19 a$ Korngrenze zur freien Oberfläche der Probe auf einen Übergang von einer monolagigen $\mathrm{zu}$ einer multilagigen Segregationszone bei einer Dotierung von 60 At.\% ppm Bi und einer Temperatur von $1123 \mathrm{~K}$. Diese Methode liefert jedoch nur sehr indirekt, unter Annahme vieler empirisch angepasster Parameter, Exzesswerte. Es ist unklar, inwieweit diese Korngrenzsegregation an der Oberfläche der Probe Rückschlüsse auf die Vorgänge im Inneren des Materials ermöglicht. Auch bei der hohen Temperatur von $1123 \mathrm{~K}$ musste aus experimentellen Gründen eine Betrachtung der Segregation des Bi an die Oberfläche der Probe gänzlich vernachlässigt werden. Der Korngrenzdiffusionskoeffizient wurde von Divinski et al. [DIVINSKI04] mit Radiotracer- 
Experimenten an polykristallinen Cu-Proben ermittelt. Diese Daten konnten jedoch nur gewonnen werden, indem unterhalb von Temperaturen gemessen wurde, die eine Löslichkeit von $\mathrm{Bi}$ in $\mathrm{Cu}$ zulassen. Daher wurden alle beobachteten Konzentrationsveränderungen der Korngrenzdiffusion zugeschrieben. Bereits im Temperaturbereich von $263-386^{\circ} \mathrm{C}$ wurde mittels AES (Auger-ElektronenSpektroskopie) ein Tracerdiffusionskoeffizient von ${ }^{207} \mathrm{Bi}$ in $\mathrm{Cu}$ zwischen $9,26 \times 10^{-14} \mathrm{~m}^{2} / \mathrm{s}$ und $1,37 \times 10^{-16} \mathrm{~m}^{2} / \mathrm{s}$ bestimmt. Innerhalb des Löslichkeitsbereichs des Phasendiagramms (Abb. 1.8) ist es nicht mehr möglich, mit dieser Methode die Volumen- und Korngrenzdiffusion getrennt zu erfassen. Höfler et al. [HöFLER93] verglichen die Diffusion von $\mathrm{Bi}$ in kompaktierten, nanokristallinen $\mathrm{Cu}$-Proben mit der Diffusion in nanokristallinen $\mathrm{Cu}-\mathrm{Bi}-\mathrm{Sch}$ ichten. Sie fanden eine zwei bis drei Größenordnungen schnellere Diffusion in den kompaktierten Proben. In Ermangelung einer höher auflösenden Messmethode konnten auch diese Beobachtungen nicht auf Prozesse auf atomarer Skala zurückgeführt werden. 



\section{Experimentelle Methoden}

In diesem Kapitel werden Messverfahren und zugrunde liegende Auswertemethoden erläutert. Bei der tomographischen Atomsonde (TAP: Tomographic Atom Probe) handelt es sich um eine Weiterentwicklung des Feld-Ionen-Mikroskopes (FIM) mit einem positionssensitiven „Time-Of-Flight“ Detektor (TOF-PSD). Aufgrund ihrer hohen Auflösung eignen sich diese Methoden sehr gut für eine mikrostrukturelle Charakterisierung von Legierungen auf subnanometer-Skala. Ausführlichere Informationen über die Feld-Ionen-Mikroskopie sind in [MILLER96] zu finden.

\subsection{Feldionenmikroskop und tomographische Atomsonde}

FIM- und TAP-Untersuchungen erfordern eine aus einem leitfähigen Probenmaterial präparierte Spitze mit einem Krümmungsradius $R$ von 20 - $100 \mathrm{~nm}$. Diese wird in einem Ultrahochvakuum von $\sim 10^{-7} \mathrm{~Pa}$ auf eine Temperatur $<100 \mathrm{~K}$ gekühlt. An die Probe kann eine stufenlos regelbare, positive Gleichspannung bis ca. $U_{0}=20 \mathrm{kV}$ angelegt werden. Der Probe auf Erdpotential gegenübergestellt ist eine Anordnung aus einer Mikrokanalplatte (MCP) und einem mit Phosphor beschichteten Leuchtschirm.

\subsubsection{Das Feldionenmikroskop (FIM)}

Die Kombination aus MCP und Leuchtschirm kann für den FIM-Modus über einen Klappmechanismus vor der Probe positioniert werden (Abb. 2.1, Pos 1). In den UHVRezipienten wird ein Bildgas, in der Regel Neon oder Helium, mit einem Partialdruck von $\sim 10^{-3} \mathrm{~Pa}$ eingelassen. 


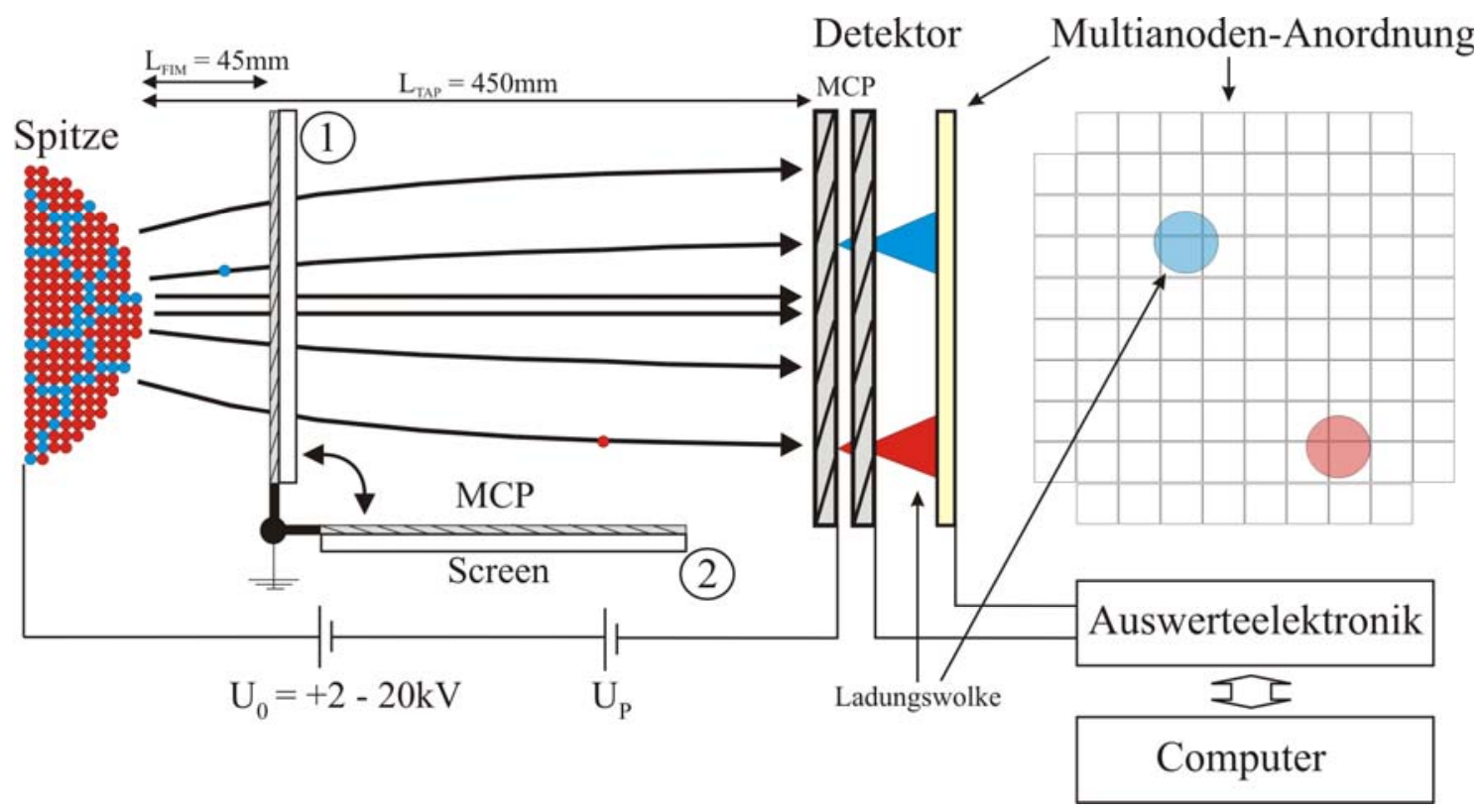

Abb. 2.1: Feldionenmikroskop mit 2-dimensionalem Detektor; (1) FIM-Modus (2) 3dAtomsonden-Modus

Aufgrund der an die Spitze angelegten Hochspannung wird am Apex der Spitze ein elektrisches Feld $E$ der Größe

$$
E=\frac{U_{0}}{\beta \cdot R}
$$

erzeugt. $\beta$ ist ein von der Form der Spitze abhängiger Geometriefaktor, dessen Wert in der Regel zwischen 5 und 7 liegt [MiLler96]. Abweichungen des Spitzenapex von einer als ideal angenommenen Kugelkappe werden durch diesen Faktor berücksichtigt. Bildgasatome werden im elektrischen Feld der Spitze polarisiert und an die Spitze herangezogen. Dort verlieren sie durch inelastische Stöße mit den Atomen der Probenoberfläche ihre kinetische Energie. Bei einer ausreichenden bildgasspezifischen Feldstärke können die Bildgasatome innerhalb eines kritischen Abstands vom Spitzenapex von $x_{k} \approx 0,5 \mathrm{~nm}$ durch einen quantenmechanischen Tunnelprozess Elektronen abgeben. Die Bildgasatome werden dann, zunächst den Feldlinien folgend, radial beschleunigt und treffen auf die MCP, wo sie Sekundärelektronen auslösen. Diese erscheinen auf dem Bildschirm als helle Leuchtpunkte und erzeugen ein stark vergrößertes Bild der Spitzenoberfläche. Aufgrund des terassenförmigen Aufbaus der Spitze bilden besonders die Kanten niedrig indizierter Ebenenscharen kristalliner 
Proben (Pole) als konzentrische Ringe ab. Korngrenzen unterbrechen die kristalline Symmetrie des FIM-Bildes und erscheinen in reinen Metallen immer mit dunklem Kontrast (Abb. 2.2).

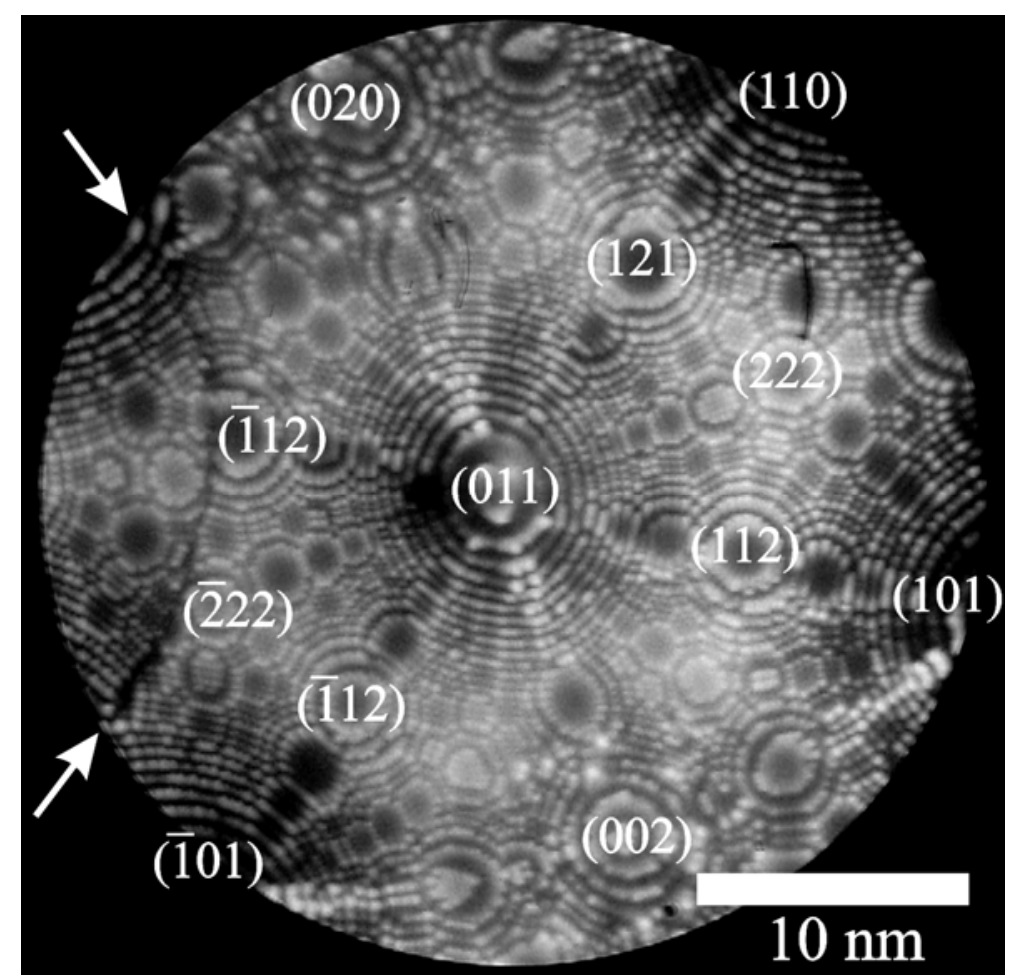

Abb. 2.2: FIM-Bild von Wolfram; die Pole niedrig indizierter Ebenenscharen sind mit Millerschen Indizes versehen; deutlich zu erkennen ist eine Korngrenze (siehe Pfeile), welche die Symmetrie des Bildes bricht.

Ein FIM-Bild entspricht ungefähr einer gnomonischen Projektion der Spitzenoberfläche mit etwa $10^{6}$-facher Vergrößerung. Die mittlere Vergrößerung am Spitzenapex $\bar{M}$ ergibt sich aus

$$
\bar{M}=\frac{L_{F I M, T A P}}{b R}
$$

wobei $R$ der Spitzenradius am Apex der Probe ist und $L_{F I M, T A P}$ die jeweiligen Abstände zum FIM-Bildschirm bzw. TAP-Detektor sind. Ein Bildkompressionsfaktor $b \approx 1,6$ (empirisch bestimmt) berücksichtigt eine Abweichung von der gnomonischen Projektion [WAGNER82]. Der Projektionspunkt wird dabei vom Spitzenapex um das 1,6 
Fache des Radius in Richtung des Schafts versetzt, damit je eine Trajektorie und eine Punktprojektion zwei gemeinsame Schnittpunkte am Ort der Probenoberfläche und am Detektor haben (Abb. 2.3).

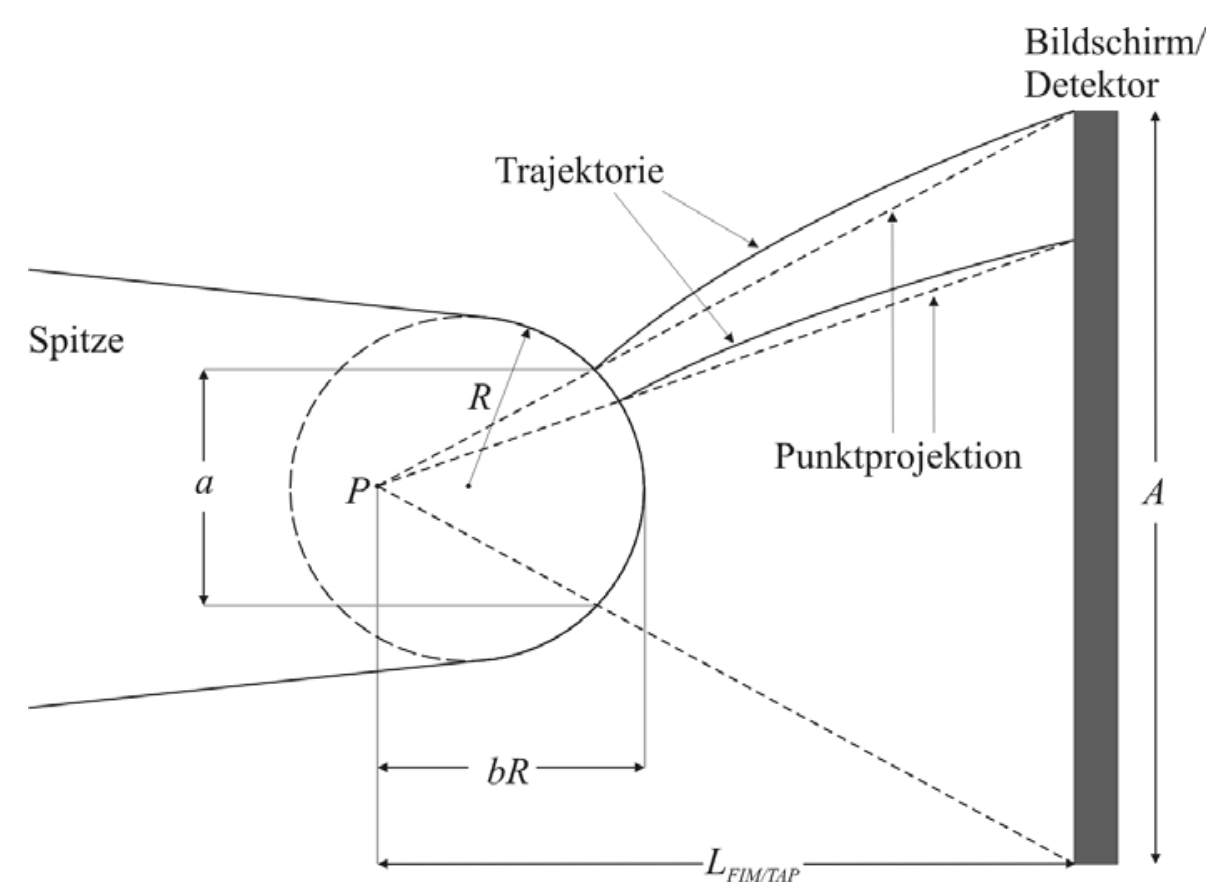

Abb. 2.3: Prinzip der zugrunde liegenden Punktprojektion des FIM und TAP

\subsubsection{Die tomographische Atomsonde (TAP)}

Durch das Umklappen des FIM-Bildschirms kann vom FIM- in den TAP-Modus umgeschaltet werden (Abb. 2.1, Pos. 2). Nachdem das Bildgas vollständig aus der Kammer entfernt ist, werden der angelegten Grundspannung $U_{0}$ Hochspannungspulse $U_{P}$, mit einer Pulslänge $t_{P}<10 \mathrm{~ns}$ und einer Frequenz von bis zu $2000 \mathrm{~Hz}$, überlagert. Die Pulse bewirken ein kontrolliertes Feldverdampfen einzelner Atome von der Spitzenoberfläche. Mit jedem Puls wird ein Kanal der Uhr gestartet, die durch das Auftreffen einen Ions auf dem Detektor wieder gestoppt wird. Aus der Flugzeit $t$ kann bei bekannter Gleichspannung $U_{0}+U_{P}$ die spezifische Masse, d.h. das Verhältnis von Masse zu Ladung $m / n$ bestimmt werden. Unter Berücksichtigung der Energieerhaltung folgt

$$
\frac{m}{n}=2 e\left(U_{0}+U_{P}\right)\left(\frac{t}{L_{T A P}}\right)^{2}
$$


Die Endgeschwindigkeit $v$ wird aufgrund der starken Lokalisation des elektrischen Feldes am Apex der Spitze kurz nach Verlassen der Spitzenoberfläche erreicht und bleibt somit über die gesamte Fluglänge konstant, wie Experimente und Simulationen bereits zeigten [Miller96], [NOWAK04]. Mit Hilfe des positionssensitiven Detektors, der aus 10 x 10 Anodenfeldern besteht, wird zu jeder gemessenen Flugzeit auch die laterale Position auf dem Detektor festgehalten. Die Vorspannung der MCP vor dem Detektor wird dazu so dimensioniert, dass die erzeugte Elektronenwolke im Mittel 3 bis 9 Anoden des Detektors trifft. Der Schwerpunkt der eintreffenden Ladungswolke $\left(x_{\text {Det }}\right.$, $\left.y_{\text {Det }}\right)$ berechnet sich durch die Verteilung der Ladungen auf dem Anodenfeld. Durch konsekutives Entfernen der Oberflächenatome erfolgt die Analyse in die Tiefe der Probe hinein. Die Tiefenskalierung in z-Richtung ergibt sich aus der Anzahl der detektierten Ionen $N$ unter Berücksichtigung der Atomvolumina $V_{A t}$ der abgetragenen Atome und der auf den Detektor projizierten, quadratischen Probenoberfläche mit der Kantenlänge $a \mathrm{zu}$

$$
d z=\frac{V_{A t} \cdot d N}{S_{D} \cdot a^{2}}
$$

wobei die Effizienz des Detektorsystems mit $S_{D} \sim 0,5 \%$ eingeht. Diese Tiefenskalierung ist jedoch nur für lokal äußerst eingeschränkte Probenbereiche korrekt. Bei einer über den Apex der Spitze ausgedehnten Analyse muss zusätzlich die Krümmung der Oberfläche in die Tiefenskalierung mit eingehen. Da der im TAP analysierte Bereich typischerweise $15 \times 15 \mathrm{~nm}^{2}$ beträgt, ist ein zusätzlicher Korrekturterm $z^{\prime}$ unverzichtbar. Aus geometrischen Überlegungen folgt

$$
z^{\prime}=R-\sqrt{R-\frac{\left(x_{D e t}^{2}+y_{D e t}^{2}\right)}{M^{2}}}
$$

Unter günstigen Messbedingungen ist nach Anwendung aller Korrekturen eine in zRichtung atomar scharfe Analyse möglich. Die Netzebenenabstände $d_{h k l}$ werden in der Rekonstruktion der 3D-Daten richtig wiedergegeben (Abb. 2.4). 


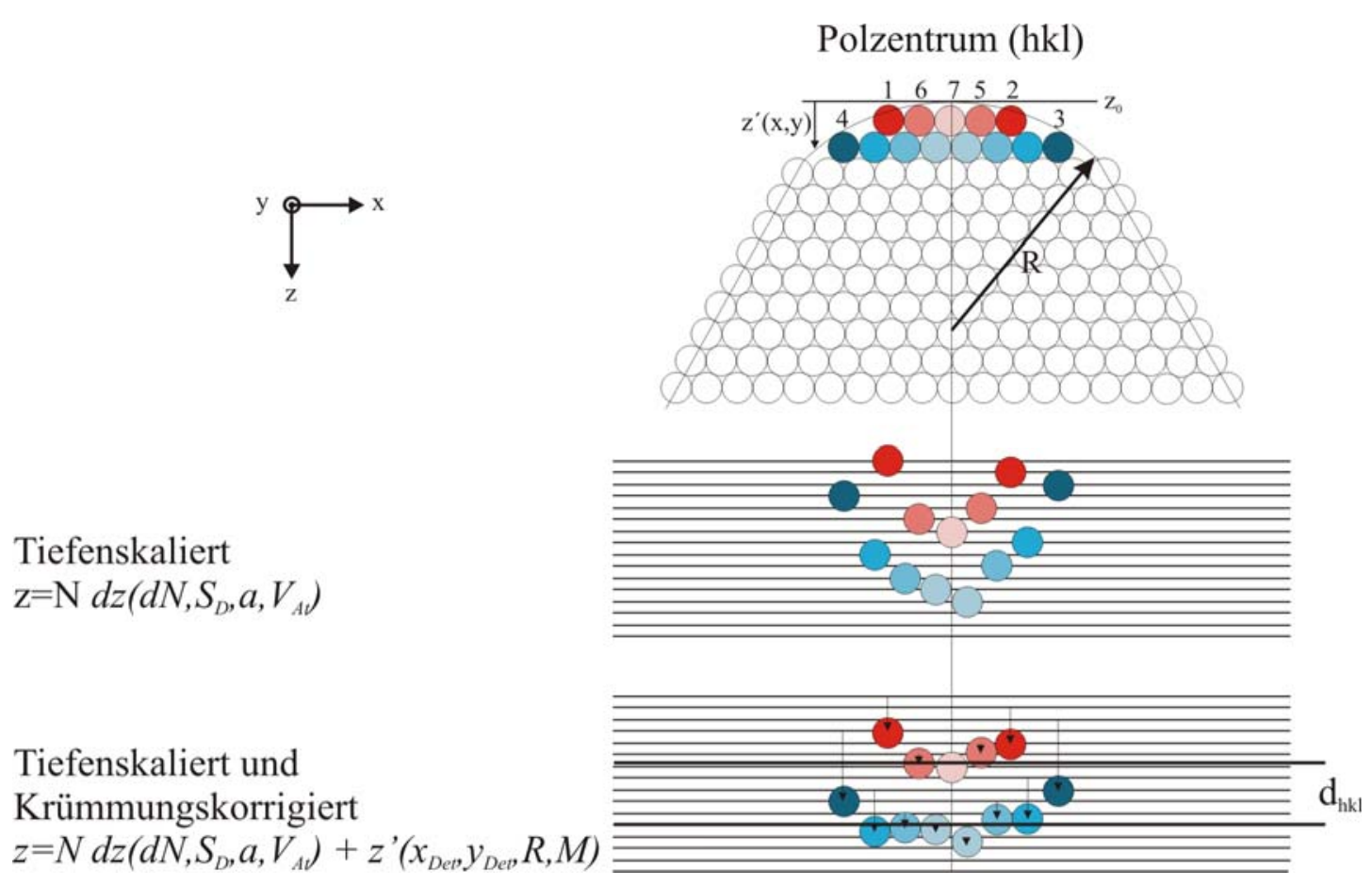

Abb. 2.4: Prinzip der Krümmungskorrektur der TAP-Daten; nach der Korrektur werden die Netzebenenabstände $d_{h k l}$ richtig wiedergegeben; die Zahlen geben die wahrscheinlichste Feldverdampfungssequenz der Atome an.

Die laterale Auflösung wird durch die Ungenauigkeit der Rückprojektion auf die Spitzenoberfläche auf $\sim 0,5 \mathrm{~nm}$ beschränkt. Alle in dieser Arbeit präsentierten 3D-TAP Daten zeigen die detektierten Atome als Kugeln, welche nicht den eigentlichen Atomgrößen entsprechen. Auf eine graphische Angabe des Fehlers der Messmethode wurde zugunsten der Übersichtlichkeit, bei den teilweise mehrere Millionen Datenpunkte umfassenden Messungen, verzichtet. Bedingt durch den Fehler der Positionierung und die von 1 verschiedene Detektionseffizienz, sind in TAP-Analysen nur dichtest gepackte Atomlagen mit Kippwinkeln $\leq 25^{\circ}$ gegen die Messrichtung auflösbar.

\subsubsection{Abbildungseffekte}

Bei phasenseparierten Proben können sich Ausscheidungen in der Matrix bilden, die sich in ihrer chemischen Zusammensetzung deutlich von der umgebenden Matrix unterscheiden. Die lokalen Feldverdampfungsfeldstärken am Ort dieser beiden Phasen 
unterscheiden sich, so dass bei stationärer Feldverdampfung ein Angleichen nur durch eine Variation des Krümmungsradius möglich ist. Damit ist die Feldionisationswahrscheinlichkeit eines Bildgasatoms in Bereichen geringerer Krümmung und somit geringerer Feldstärke kleiner, als in Bereichen größerer Krümmung mit höherer Feldstärke. Die hervorstehenden Phasen werden deshalb im FIM-Modus mit einem hellen, die zurückweichenden Phasen mit einem dunklen Kontrast abgebildet. Bedingt durch die auftretenden Projektionseffekte können dann z.B. Teilchengrößen nur mit einer gewissen Ungenauigkeit angegeben werden (Abb. 2.5). Dies bezeichnet man als „local magnification effect“ [VURPILLOT00].
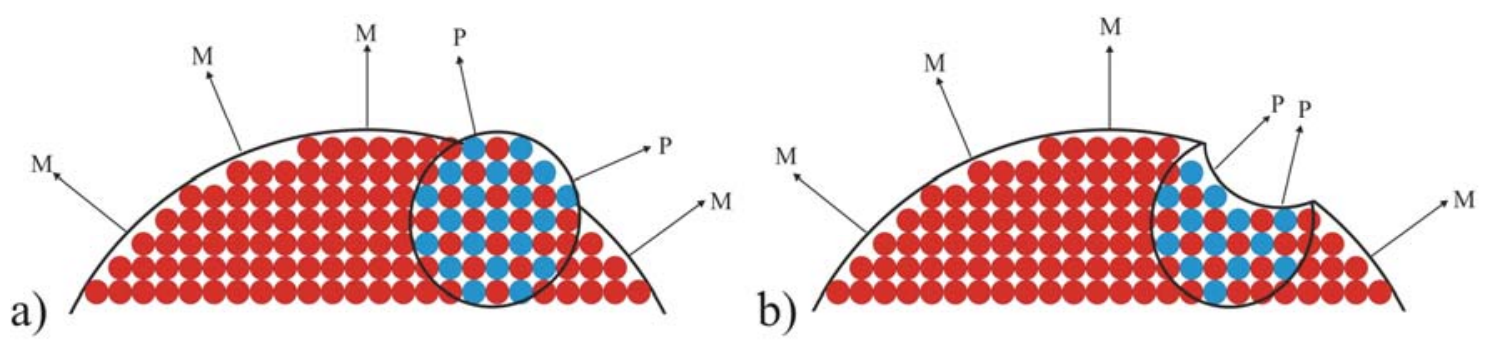

Abb.2.5 Unterschiedliche Projektionseffekte verschiedener Phasen; a) Ausgeschiedene Phase hat eine höhere Feldverdampfungsfeldstärke (heller Kontrast im FIM-Bild), b) Ausgeschiedene Phase hat eine niedrigerer Feldverdampfungsfeldstärke als die Matrix (dunkler Kontrast im FIM-Bild).

\subsubsection{Auswertungsmethoden der Atomsondendaten}

Die dreidimensionale, chemisch aufgelöste Analyse der Probe liefert eine Punktmenge von Atomen. Diese wird über die Auswertungssoftware AVS/UNIRAS visualisiert. Zusätzlich zu bestehenden Funktionen können Analysealgorithmen durch den Benutzer eingebunden werden. Eine Übersicht über aktuelle Analysefunktionen findet sich in [WOLDE01].

Ein besonderer Schwerpunkt liegt auf Leiterdiagrammen. Dabei wird die kumulative Anzahl der Ionen einer Komponente $B$ gegen die Anzahl aller Ionen $A+B$ aufgetragen. Typischerweise wird für diese Auswertung ein Pfad entlang eines Zylinders durch die Punktmenge von Atomen vorgegeben. Um eine Auskunft über lokale Konzentrationen 
an Grenzflächen zu erhalten wird der Zylinder so positioniert, dass er diese möglichst senkrecht schneidet. Die Standardabweichung $\sigma$ des gemessenen Konzentrationswertes ergibt sich aus

$$
\sigma=\sqrt{\frac{c_{o}\left(1-c_{0}\right)}{A-1}}
$$

wobei $c_{0}$ die über den Analyse-Zylinder gemittelte Konzentration und $A$ die Anzahl der beteiligten Atome ist. Nach der Binomialstatistik liegen 95,5\% aller Messwerte einer homogenen Atomverteilung in einem 2 $\sigma$-Intervall [KRENGEL00]. Auf diese Art lässt sich feststellen, ob eine signifikante Konzentrationsänderung vorliegt.

Um zu überprüfen, ob eine Komponente B homogen oder heterogen in einer Probe verteilt ist, kann ein $\chi^{2}-$ Test durchgeführt werden. Der Wert berechnet sich aus

$$
\chi^{2}=\sum_{j=1}^{k} \frac{\left(S_{j}-S p_{j}\right)^{2}}{S p_{j}}
$$

und ist ein Maß für die Abweichung einer gemessenen Verteilung von einer Binomialverteilung [KRENGEL00], welche einer statistischen Verteilung der Komponente B entspricht. Das rekonstruierte Volumen wird wiederum in geeignete Blöcke unterteilt, wobei $S_{j}$ die Anzahl der Blöcke, in denen die Ionensorte B $j$-mal auftritt, $S$ die Gesamtzahl der Blöcke, $k$ die Anzahl der Klassen und $p_{j}$ die Wahrscheinlichkeit gemäß der Binomialverteilung dafür ist, dass ein Block in $j$ fällt. Der berechnete $\chi^{2}$-Wert unterliegt selbst einer Dichteverteilung, deren zugehörige Wahrscheinlichkeitswerte in der Literatur tabelliert sind [BRONSTEIN95]. Der $\chi^{2}$-Test wird nur in zwei Fällen als signifikant betrachtet. Wenn die ermittelte Wahrscheinlichkeit größer als $95 \%$ ist, kann eine homogene Verteilung der Komponente B angenommen werden. Ist die Wahrscheinlichkeit kleiner als $5 \%$, so wird eine heterogene Verteilung dieser Komponente in der Probe angenommen. 


\subsubsection{Parameter für FIM- und TAP-Analysen}

Eine statistisch aussagekräftige Auswertung der TAP-Daten kann nur für eine hinreichend große Anzahl (> 50000) von detektierten Atomen durchgeführt werden. Durch eine geeignete Wahl der Meßparameter muss sichergestellt werden, dass die aus dem Probenmaterial präparierte Spitze unter dem Einfluss des angelegten elektrischen Feldes und den damit verbundenen mechanischen Spannungen von $\sim 10^{10} \mathrm{~Pa}$ nicht frühzeitig abreißt. Die wesentlichen Parameter sind hierbei die Temperatur und das Pulsverhältnis, als das Verhältnis von Pulsspannung zu Grundspannung $U_{P} / U_{0}$. Eine höhere Temperatur $\sim 60 \mathrm{~K}$ und ein niedrigeres Pulsverhältnis $\sim 0,15$ sind zur Vermeidung eines Abrisses geeignet, jedoch resultiert dies in einer unkontrollierten Feldverdampfung der leichter zu verdampfenden Komponente. Die Konzentration dieser Komponente wird dann unterschätzt. Häufiger begünstigt die Wahl einer möglichst tiefen Temperatur $\sim 20 \mathrm{~K}$ die Angleichung der Feldverdampfungsfeldstärken der Komponenten [MüLLER69]. Zusätzlich wirkt ein hohes Pulsverhältnis einer unkontrollierten Feldverdampfung entgegen. Dies ist besonders bei den gesputterten $\mathrm{Ag} / \mathrm{Ni}$ Schichten wichtig, da sie Multilagen mit Atomen stark unterschiedlicher Feldverdampfungsfeldstärken darstellen. Bei diesen Proben erwies es sich weiterhin als sinnvoll, die Geschwindigkeit der Analyse zu reduzieren und die angelegte Spannung beim Erreichen der nächsten Schicht möglichst schnell einzuregeln ${ }^{2}$.

\footnotetext{
2 Besonders beim Durchtritt von einer Schicht mit hoher zu niedriger Feldverdampfungsfeldstärke ergeben sich, wie später gezeigt wird, im System Ag/Ni Probleme mit unkontrollierter Feldverdampfung.
} 


\subsection{Weitere Analysemethoden}

Es ist offensichtlich, dass die Durchführung quantitativer Untersuchungen auf atomarer Skala bei geringsten Konzentrationen das Zusammenspiel mehrerer Analysemethoden erforderlich macht. Die weiteren Arbeit angewandten Analysemethoden werden im Folgenden kurz erläutert.

\subsubsection{Elektronenmikroskopie}

Neben der tomographischen Atomsonde wurde für die Charakterisierung der untersuchten Proben ein Rasterelektronenmikroskop des Typs „Supra 25“ der Firma Carl Zeiss mit einem angeschlossenen EDX-System des Typs „System SIX“ der Firma Noran zur quantitativen Analyse eingesetzt.

Die genaue Untersuchung der Spitzengeometrie der hergestellten FIM/TAP Proben wurde mit Hilfe eines Transmissionselektronenmikroskops des Typs „EM $400 \mathrm{~T}^{\text {“ der }}$ Firma Philips durchgeführt. Hierzu wurde ein auf FIM/TAP Spitze umgebauter Probenhalter benutzt. Unterhalb eines Spitzendurchmessers von $100 \mathrm{~nm}$ bietet das mit einer $\mathrm{LaB}_{6}$-Kathode ausgestattete Gerät bei der maximalen Beschleunigungsspannung von $120 \mathrm{kV}$ die Möglichkeit der Transmission durch die Probe. Mit Hilfe des Orientierungskontrastes können einzelne Körner sichtbar gemacht werden und aus Beugungsaufnahmen Kristallorientierungen bestimmt werden. Hochauflösende Bilder einzelner Korngrenzen wurden mit einem Transmissionselektronenmikroskop des Typs „CM 200“ der Firma Philips im Labor für Elektronenmikroskopie der Universität Karlsruhe durchgeführt. 


\subsubsection{ICP-OES}

Die optische Emissionsspektroskopie ist eine der verbreitesten Methoden der analytischen Chemie. Durch die Einführung eines induktiv gekoppelten Hochfrequenzplasmas als Strahlungsquelle (Inductively Coupled Plasma Optical Emission Spectroscopy, ICP-OES) wurde eine wesentliche Erweiterung der Methodik erzielt, wobei Nachweisgrenzen im $n g / m l$ Bereich erreicht werden können. Die Probe wird als Aerosol in das Plasma gegeben, wobei die Atome Photonen einer elementspezifischen Wellenlänge emittieren. Die Erfassung des Emissionsspektrums erfolgt durch ein Rowland-Spektrometer. Durch eine Kalibrierung gegen bekannte Standards ermöglicht diese Methode eine quantitative Analyse der Probenzusammensetzung. Abhängig vom Probenmaterial kann die Herstellung eines geeigneten Aerosols durch Lösemittel problematisch sein und birgt, darüber hinaus, ein großes Risiko der Probenkontamination. Es bietet sich im Fall metallischer Proben die Möglichkeit an, die Probe direkt durch eine elektrische Entladung zu zerstäuben und diese dem Plasma zuzuführen. Hierzu wird zwischen der Probe (Kathode) und einer Gegenelektrode aus Wolfram (Anode) eine elektrische Entladung gezündet. Diese ist üblicherweise unipolar, woraus resultiert, dass nur Probenmaterial abgetragen wird. Diese Vorgehensweise schließt eine Kontamination weitestgehend aus. Mittels dieser Methode wurde die Nominalkonzentration der Cu-Bi Bikristalle bestimmt.

\subsubsection{Röntgendiffraktometrie (XRD)}

An einem Vierkreis-Diffraktometer des Typs „X'Pert“ der Firma Philips wurden Texturmessungen durchgeführt, um die laterale kristallographische Kornorientierung der co-evaporierten Ag-Ni Proben zu untersuchen. Bei der eingesetzten Röntgenröhre handelte es sich um einen Co-Emitter der Wellenlänge $\lambda=1,7903 \AA$ (Co-K $\alpha)$. Für die Darstellung der gewonnenen Ergebnisse wurden für diese Arbeit Polfiguren gewählt. 



\section{Probenpräparation}

In dieser Arbeit wurden Proben aus drei verschiedenen Herstellungsverfahren untersucht. In diesem Kapitel werden diese Verfahren und die zugrunde liegenden Parameter vorgestellt und erläutert. Es wird eine neue Methode der Präparation von FIM/TAP Probenspitzen mit Hilfe der „Focused Ion Beam“-Technik eingeführt. Durch einen Mikromanipulator kann mittels der „Lift-Out“-Technik zusätzlich eine Zielpräparation an wohldefinierten Korngrenzen durchgeführt werden.

\subsection{Sputterdeposition}

Eine Anforderung für die Untersuchung der Korngrenzsegregation auf atomarer Skala ist zunächst die Herstellung von TAP-Proben mit einem nanokristallinen Gefüge. Solche Proben bieten eine hinreichend große Wahrscheinlichkeit der Analyse von Korngrenzen ohne die Notwendigkeit einer Zielpräparation. Ag-Ni bzw. Cu-Bi Schichten wurden durch Ar-Ionen-Sputtern auf Wolfram-Substratspitzen deponiert. Aufgrund der starken Krümmung des Substrates ist mit einem nanokristallinen, kolumnaren Wachstum der Schicht zu rechnen [LANG02]. W-Drähte mit einem Querschnitt von $70 \mu \mathrm{m}$ wurden zuvor mit einem Standardverfahren elektrolytisch zu Spitzen gedünnt ${ }^{3}$. Anschließend wurden diese im FIM bis zu einer Spannung von $12-$ $13 \mathrm{kV}$ entwickelt um eine saubere, ideal kugelkappenförmige Oberfläche mit definiertem Krümmungsradius von $38-42 \mathrm{~nm}$ am Apex zu gewährleisten.

Die Beschichtung der Spitzen wurde in einer eigens dafür konzipierten UHV-Anlage durchgeführt [SCHLEIWIES01], die das gleichzeitige Beschichten von bis zu vier Spitzen von je einem von vier unterschiedlichen Targets erlaubt (Abb. 3.1). Es muss eine ausreichende mechanische Stabilität der Schichten gegenüber den während der Analyse auftretenden Zugspannungen bei hohen elektrischen Feldern erzielt werden. Daher ist die vorherige Reinigung der Spitzenoberfläche durch einen abgeschwächten Ar-Strahl unerlässlich [ALKASSAB95]. Die speziell hierfür konzipierte Sputterkammer ermöglicht

\footnotetext{
${ }^{3}$ Elektrolytische Dünnung in einer 2 molaren Natronlauge bei einer Wechselspannung von bis zu $12 \mathrm{~V}$.
} 
durch eine einfache Rotation des Probenträgers beide Modi der Substratreinigung und des Beschichtens mit einer Kaufmann Quelle.

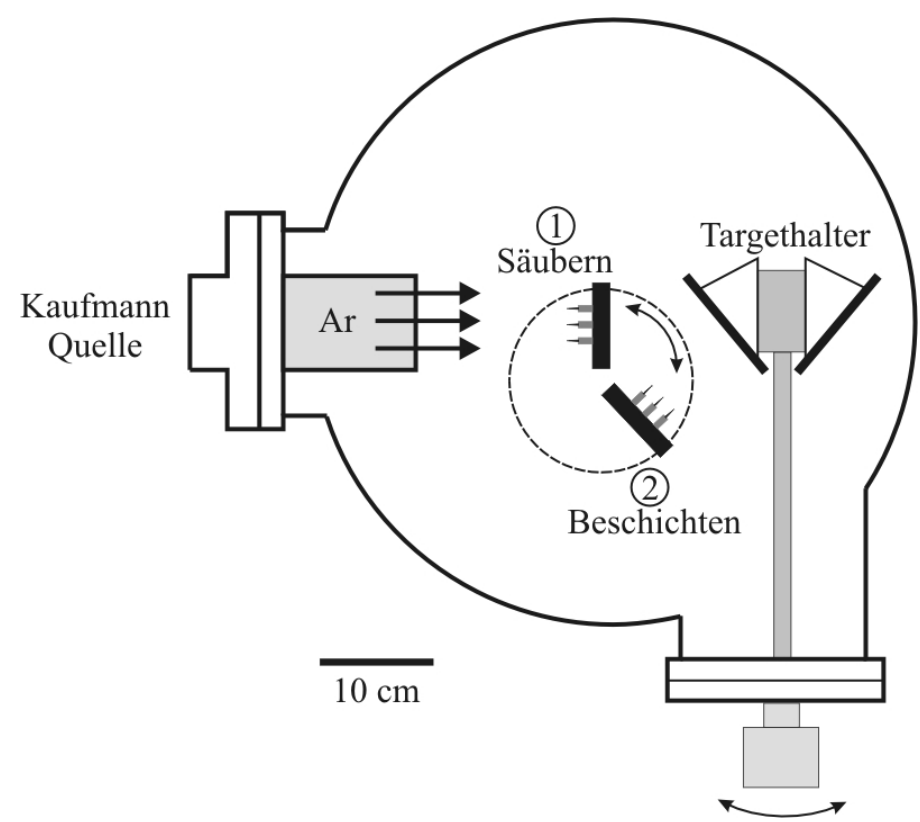

Abb. 3.1: Schematische Darstellung der verwendeten Sputterkammer; säubern der WSubstratspitzen an Pos. 1, Beschichten vom gewählten Target an Pos. 2

Für die Untersuchung des Ag-Ni-Legierungssystems wurden mit dieser Anlage zwei bis drei Doppelschichten von zwei wechselnden Targets direkt auf die W-Spitze gesputtert. Idealerweise sollte die Feldverdampfungsfeldstärke der Schichten zum Substrat hin zunehmen ${ }^{4}$. Daher wurde Ni immer als erste Schicht deponiert. Diese Idealstruktur konnte aufgrund der Doppelschichten mit stark alternierenden Feldverdampfungsfeldstärken von $\mathrm{Ag}(24 \mathrm{~V} / \mathrm{nm})$ und $\mathrm{Ni}(35 \mathrm{~V} / \mathrm{nm})$ nicht eingehalten werden ${ }^{5}$.

Im Fall des $\mathrm{Cu}$-Bi-Legierungssystems wurde eine nanokristalline $\mathrm{Cu}-\mathrm{Bi}$ Schicht von einem einzelnen Komposit-Target gesputtert. Dabei kamen drei verschiedene Targettypen zum Einsatz. In einem ersten Versuch wurde von einem Legierungstarget der Zusammensetzung $\mathrm{Cu}-1 \mathrm{At}$ \% Bi gesputtert. Während des induktiven

\footnotetext{
${ }^{4}$ Eine unkontrollierte Feldverdampfung von Atomen beim Durchtritt in die nächste Schicht wird dadurch vermieden.

${ }^{5}$ Die auftretenden Artefakte konnten, wie in Abschnitt 2.1.5 beschrieben, minimiert werden.
} 
Aufschmelzens unter einer Ar-Schutzgasatmosphäre bildeten sich millimetergroße BiEinschlüsse innerhalb der Cu-Matrix (Abb. 3.2a). Es zeigte sich in TAP-Messungen jedoch keine signifikante Anreicherung von $\mathrm{Bi}$ in den deponierten Schichten dieses Targets. Eine Erhöhung der Bi-Konzentration war aufgrund der ausgeprägten Mischungslücke des $\mathrm{Cu}$-Bi-Legierungssystems auf diesem Wege technisch nicht realisierbar. Um eine größere Bi-Fläche auf dem Target bereitzustellen wurde ein reines Bi-Target mit kreisrunden $\mathrm{Cu}$-Blech versehen, welches unter einem Winkel $\alpha$ eingeschnitten wurde (Abb. 3.2b). Unter Annahme eines 1:1 Übertrags kann aus den während des Sputtervorgangs angebotenen Flächenanteilen des Targets über die Beziehung

$$
c_{B i}=\frac{\alpha}{360^{\circ}}
$$

die auf dem Substrat zu erwartende Bi-Konzentration in der deponierten Schicht berechnet werden. Schließlich, um eine geringere Inhomogenität des Targets zu erreichen wurden durch ein lithographisches Verfahren Gitter (Stegbreite 1,4 mm) mit einer variablen Transparenz aus einem reinen Cu-Blech $(\mathrm{Cu}$ 99,999\%, $125 \mu \mathrm{m}$ Stärke) geätzt $^{6}$. Dieses wurde vor einem Bi-Target (Bi 99,5\%) angebracht (schematisch Abb. 3.2c), technische Realisierung (Abb. 3.3).
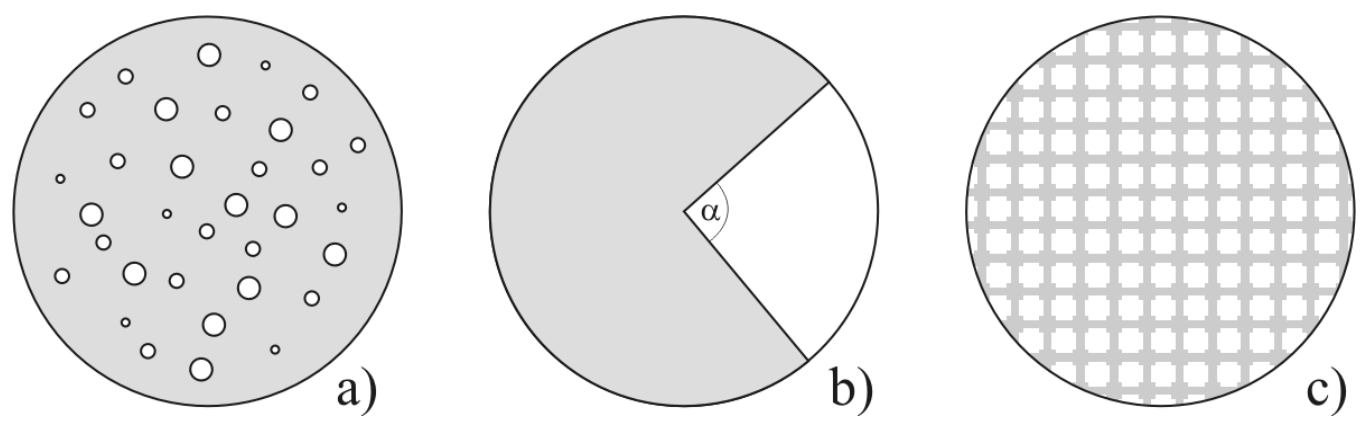

Abb. 3.2: Erprobte Targettypen;Cu: grau, Bi: weiss; a) Cu-1 At.\% Bi Legierungstarget; b) Target mit variablen Cu/Bi Flächenanteilen; c) Target mit Cu-Gitter (50\% Transparenz)

\footnotetext{
${ }^{6}$ Fotolack „Positiv 20“ vom Hersteller Kontakt Chemie, beidseitiges Ätzen nach UV-Belichtung durch eine gesättigte Lösung Eisen-III-Chlorid
} 


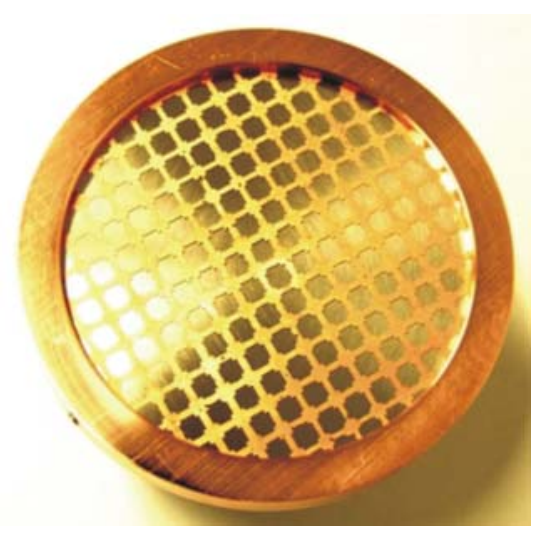

Abb. 3.3: Technische Realisierung eines Bi-Targets mit Cu-Gitter (50\% Transparenz)

Die Haftung zwischen der Cu-Bi-Schicht und dem W-Substrat konnte durch eine CoSchicht deutlich verbessert werden.

\subsection{Co-Evaporation}

In Rahmen dieser Arbeit wurden auch bulk-nanokristalline Ag-Ni-Proben untersucht, die aus der Arbeitsgruppe von Prof. Birringer (Universität des Saarlandes) bezogen wurden. Es ist erstmalig erfolgreich gelungen, FIM/TAP Proben zu präparieren, welche durch einen Kondensations- und Kompaktierungsprozess hergestellt wurden. Die einzelnen Schritte der Präparation werden im Folgenden beschrieben.

Der experimentelle Aufbau besteht aus zwei thermischen Verdampfern, die je Ag und $\mathrm{Ni}$ enthalten, und einem rotierenden Kühlfinger in einer UHV-Kammer. Ein Leckventil oberhalb der Verdampfungstiegel sorgt für einen kontinuierlichen Fluss von He in Richtung des Kühlfingers (Abb. 3.4). Dieser wird so reguliert, dass sich ein Gesamtdruck von 1 mbar in der Kammer einstellt. Nach einer Evaporationszeit von 0,5 bis $2 h$ ist ausreichend Material am stickstoffgekühlten Kühlfinger kondensiert. Über einen Abstreifer kann das Material in situ einem zweistufigen Verdichter zugeführt werden. In der letzten Stufe wird die Probe mit einem Druck von 1 GPa zu einer Scheibe der Dicke von 150-200 $\mu m$ verdichtet (Abb. 3.5). Die Koaleszensrate der Atome in der Gasphase bestimmt, im Fall nur einer verdampften Komponente, direkt die Korngröße. Um ein weiteres Kornwachstum auf dem Kühlfinger zu verhindern können zusätzlich weitere Legierungselemente zugegeben werden, die zu 
einer Benetzung der Korngrenzen führen [KonRAD96]. Die Koaleszensrate kann direkt über die Verdampfungsraten von $\mathrm{Ag}$ und Ni reguliert werden.

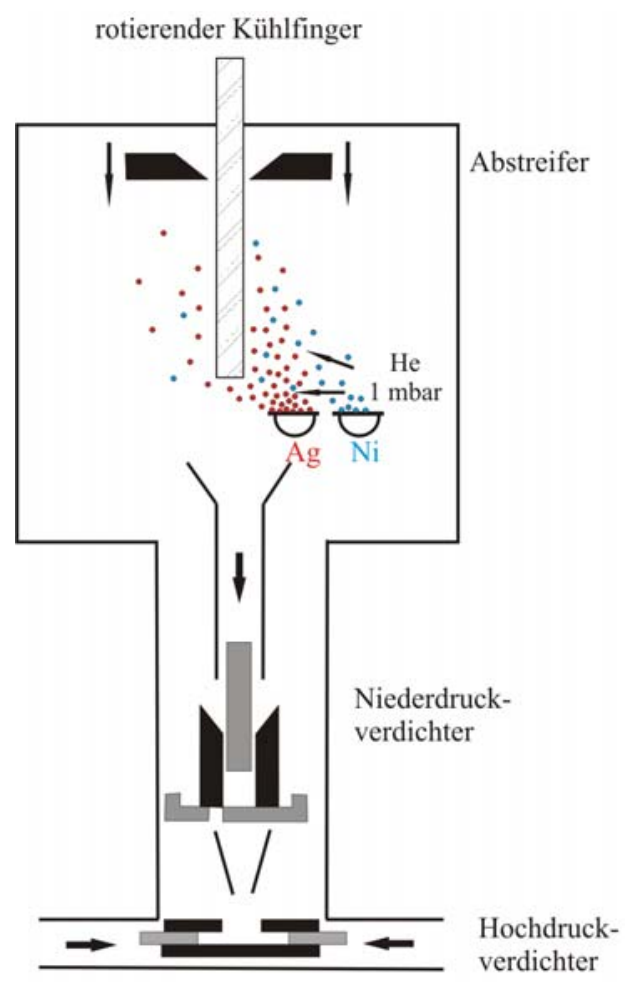

Abb. 3.4: Experimenteller Aufbau der Kondensationskammer mit zweistufigem Verdichter

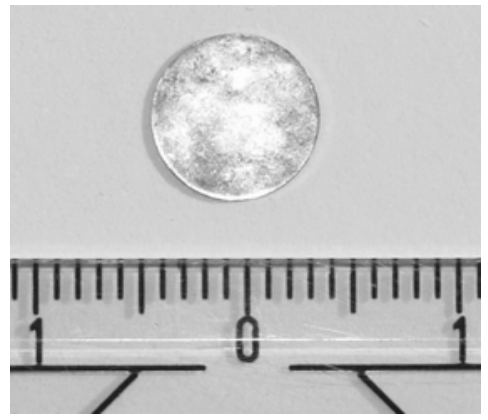

Abb. 3.5: Kompaktierte Ag-Ni Probe; Durchmesser typischerweise $8 \mathrm{~mm}$, Dicke $150-200 \mu m$

Die scheibenförmigen Ag-Ni-Proben wurden mit einer Funkenerosionsmaschine senkrecht zur Pressrichtung in $200 \mu \mathrm{m}$ breite Streifen geschnitten und elektrolytisch zu FIM/TAP Spitzen gedünnt. Eine Elektrolytmischung aus $\mathrm{HClO}_{4}$ und $\mathrm{CH}_{3} \mathrm{COOH}$ im Volumenverhältnis 1:10 lieferte bei Gleichspannungen von 1,9 bis 2,2 $\mathrm{V}$ gute Ergebnisse. 


\subsection{Präparation Bi dotierter $219 a$ Bikristalle}

Die Untersuchung der Segregation an definierten Korngrenzen erfordert Bikristalline $\mathrm{Cu}$-Proben, die in Zusammenarbeit mit Dr. Gabriel Alejandro López vom Max-PlanckInstitut für Metallforschung in Stuttgart hergestellt wurden. Diese wurden anschließend homogen mit Spuren von Bi dotiert. Die komplexen Arbeitsschritte vom Schmelzen der Legierung bis zur Fertigung einer FIM/TAP-Probe werden in den folgenden Abschnitten erläutert.

\subsubsection{Herstellung und Dotierung}

Es wurden zunächst Bikristalline Cu-Proben mit dem Bridgeman-Verfahren hergestellt. Dabei wird die Schmelzzone langsam unter einer Ar-Schutzgasatmosphäre durch polykristallines Kupfer (Reinheit 99,999\%) gezogen. Zwei geeignet orientierte Einkristalle dienen dabei als Impfkristalle und sorgen für einen Orientierungsübertrag (Abb. 3.6). Bei der $\Sigma 19 a$ Korngrenze handelt es sich um eine symmetrische Kippkorngrenze, deren Struktur eindeutig mit der Koinzidenzgitter-Theorie beschrieben werden kann.

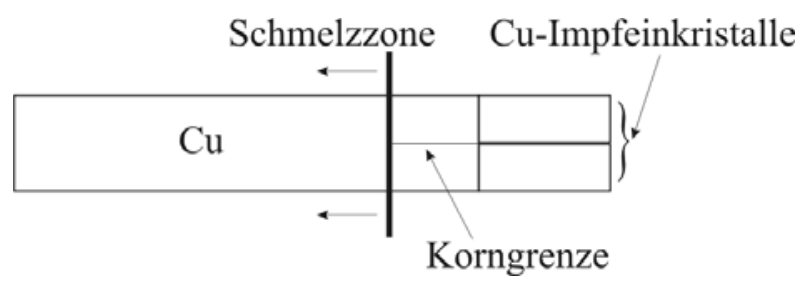

Abb. 3.6: Bridgeman-Verfahren zur Bikristallherstellung

Die Bikristalle wurden, zusammen mit einem $\mathrm{Cu}$-Einkristall, zur Dotierung in evakuierte Quarzglasampullen eingeschweißt. Eine entmischte $\mathrm{Cu}-1 \mathrm{At}$ \% \% BLegierung diente als Bi-Quelle. Die Verwendung einer solchen Quelle verhindert das unkontrollierte Verdampfen des $\mathrm{Bi}$ bei den für eine ausreichende Löslichkeit in $\mathrm{Cu}$ benötigten Temperaturen (siehe Abschnitt 1.2.2). Der Bi-Gehalt im Bikristall wird 
durch die äußeren Abmessungen der Bikristalle sowie die Dotierzeit bestimmt ${ }^{7}$. Eine mehrtätige Temperaturbehandlung bei $950{ }^{\circ} \mathrm{C}$ sorgt für eine homogene Verteilung des Bi. Durch eine Segregationsbehandlung wurde eine Gleichgewichtssegregation an der Korngrenze eingestellt. Es wurden ursprünglich zwei Probenserien mit Konzentrationen von 50 und $100 \mathrm{ppm} \mathrm{Bi}$ angestrebt. Durch spontane Sprödbrüche an der Korngrenze nach der Segregationsbehandlung war jedoch keine weitere Verarbeitung der höher dotierten Bikristalle mehr möglich. Alle Schritte der Präparation für beide Probenserien sind in Abbildung 3.7 schematisch dargestellt.

Die Nominalkonzentrationen der dotierten Bikristalle konnten anhand des ReferenzEinkristalls mit Hilfe der ICP-OES zu $40 \pm 4$ ppm Bi bzw. $120 \pm 3$ ppm Bi bestimmt werden. Weitere Informationen zur Probenherstellung finden sich in [LÓPEZ04].

Quarzglaskapsel für Dotierung bei $880{ }^{\circ} \mathrm{C}$ für $24 \mathrm{~h} / 65,5 \mathrm{~h}$

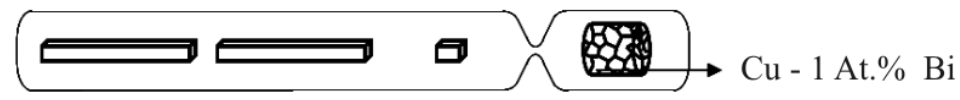

Quarzglaskapsel für Homogenisierung bei $950{ }^{\circ} \mathrm{C}$ für $10 \mathrm{~d}$

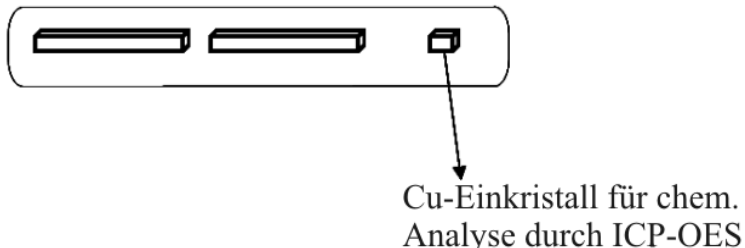

Segregationsbehandlung bei $850^{\circ} \mathrm{C}$ für $12 \mathrm{~h}$

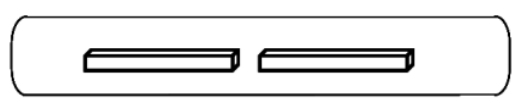

Abb. 3.7: Dotierung, Homogenisierung und Segregationsbehandlung der Bikristalle mit Abmessungen $3 \times 3 \times 15 \mathrm{~mm}^{3}$; Dotierzeiten für angestrebte $50 \mathrm{bzw} .100 \mathrm{ppm}$ Bi waren $24 \mathrm{~h} \mathrm{bzw.65,5}$.

\footnotetext{
${ }^{7}$ Die Dotierzeiten für die gewünschten Werte wurden bei den Abmessungen der Bikristalle und der gewählten Temperatur zuvor empirisch bestimmt.
} 


\subsubsection{Vorgehensweisen zur Spitzenpräparation}

Um Korngrenzen mit Hilfe der 3D-Atomsondentomographie zu untersuchen wurden in bisherigen Arbeiten möglichst nanokristalline Materialien untersucht, bei denen die statistische Wahrscheinlichkeit, eine Korngrenze am Apex einer elektrolytisch gedünnten Spitze zu finden, ausreichend groß ist. Diese Vorgehensweise scheitert im Fall des untersuchten $\mathrm{Cu}$-Bi-Bikristalls mit nur einer einzelnen Korngrenze. Es wurde in enger Zusammenarbeit mit Dr. Fabian Pérez-Willard vom LEM (Labor für Elektronenmikroskopie) Karlsruhe ein neues Verfahren entwickelt, bei dem die $\mathrm{Cu}$-BiBikristalle mit Hilfe der „Lift-Out“ Technik in einem „Focused Ion Beam“ (FIB)-Gerät zu TAP/FIM Proben präpariert wurden. Die Präparation wurde in einem Gerät der Firma Carl Zeiss, Typ 1540 XB, durchgeführt. Dieses Gerät besitzt für den Simultanbetrieb zwei Rastereinheiten („,dual beam“) für einen Elektronen- und Ionenstrahl. Die Strahlbündel treten aus zueinander verkippten Säulen aus (Abb. 3.8).

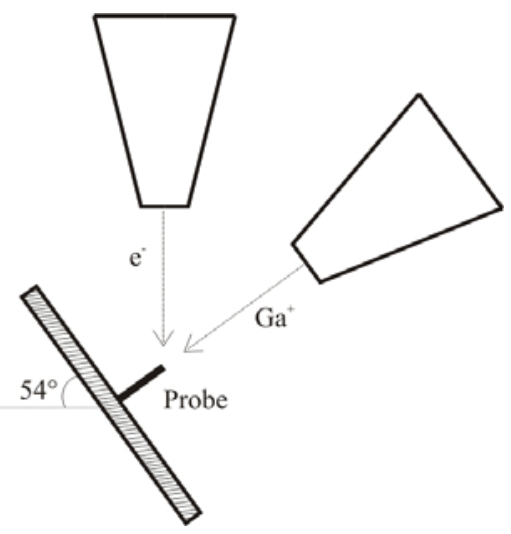

Abb. 3.8: Schematische Darstellung des verwendeten FIB-Gerätes; der Kippwinkel der Säulen ist nicht variabel und beträgt $54^{\circ}$.

Die polierte und $2-3 s$ mit $\mathrm{HNO}_{3}(65 \%)$ angeätzte Stirnfläche eines Bikristalls ist im Rückstreuelektronenbild deutlich $\mathrm{zu}$ erkennen (Abb. 3.9a). Die Ätzspuren beider Bikristallhälften entsprechen mit einem gegenseitigen Winkel von $153,5^{\circ}$ dem erwarteten Kippwinkel der $\Sigma 19 a$ Korngrenze. Abbildung $3.9 \mathrm{~b}$ zeigt eine um $45^{\circ}$ gekippte Ansicht der Korngrenze. Es bildet sich durch ein anisotropes Ätzverhalten eine Stufe, die in ungünstigen Fällen einige Mikrometer breit sein kann. Mit einem 
streifenden Einfall des $\mathrm{Ga}^{+}$- Ionenstrahls muss diese zunächst nivelliert werden (Abb. $3.9 \mathrm{c})$.

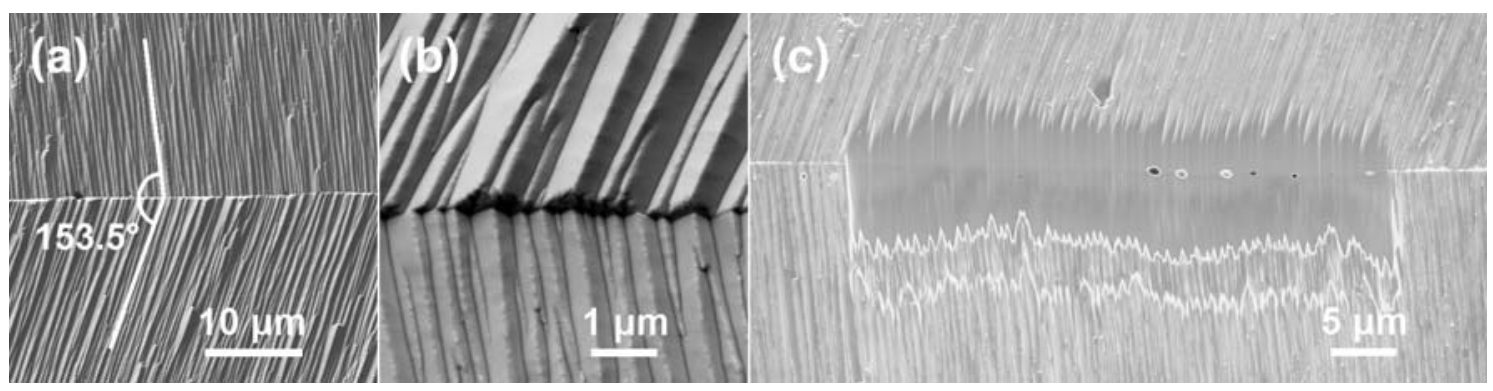

Abb. 3.9: Vorbereitung der Korngrenze; a) Übersicht der KG mit Kippwinkel; b) um $45^{\circ}$ gekippte Ansicht; c) nivellierte KG nach streifendem Ga+ - Beschuss

Eine Pt-Abscheidung markiert und schützt die Korngrenze für die weiteren Bearbeitungsschritte (Abb. 3.10a). Um die markierte Korngrenze herum wird nun beidseitig ein ca. $15 \mu \mathrm{m}$ tiefer und $20 \mu \mathrm{m}$ breiter Graben mit einem Gefälle von ca. $45^{\circ}$, in Analogie zur TEM-Lamellenpräparation ([OVERWIJK93], [GIANNUZZI99]), geschnitten. Durch vorsichtige Schnitte mit abnehmendem $\mathrm{Ga}^{+}$-Strahlstrom nähert man sich der Korngrenze immer weiter an. Ein Stück der so entstandenen Lamelle wird dann durch zwei seitliche Schnitte abgetrennt und verbleibt als freistehender Pfosten mit den Abmessungen $2 \times 2 \mu^{2}$. Der Mikromanipulator wird nun auf das herauszuhebende Probenstück aufgesetzt und durch eine gezielte Pt-Abscheidung verbunden (Abb. 3.10b). An der Verankerungslinie wird es dann durch einen weiteren Schnitt vollständig abgetrennt, so dass es mit dem Mikromanipulator abtransportiert werden kann (Abb. 3.10c-d). Bis zu fünf Probenstücke können auf diesem Wege aus einer Lamelle geschnitten werden. Das Stück wird auf einem zuvor präparierten Träger aus Wolfram durch eine Pt-Abscheidung befestigt und dann durch einen zweiten Schnitt von der Mikromanipulatorspitze abgetrennt (Abb. 3.10e). 


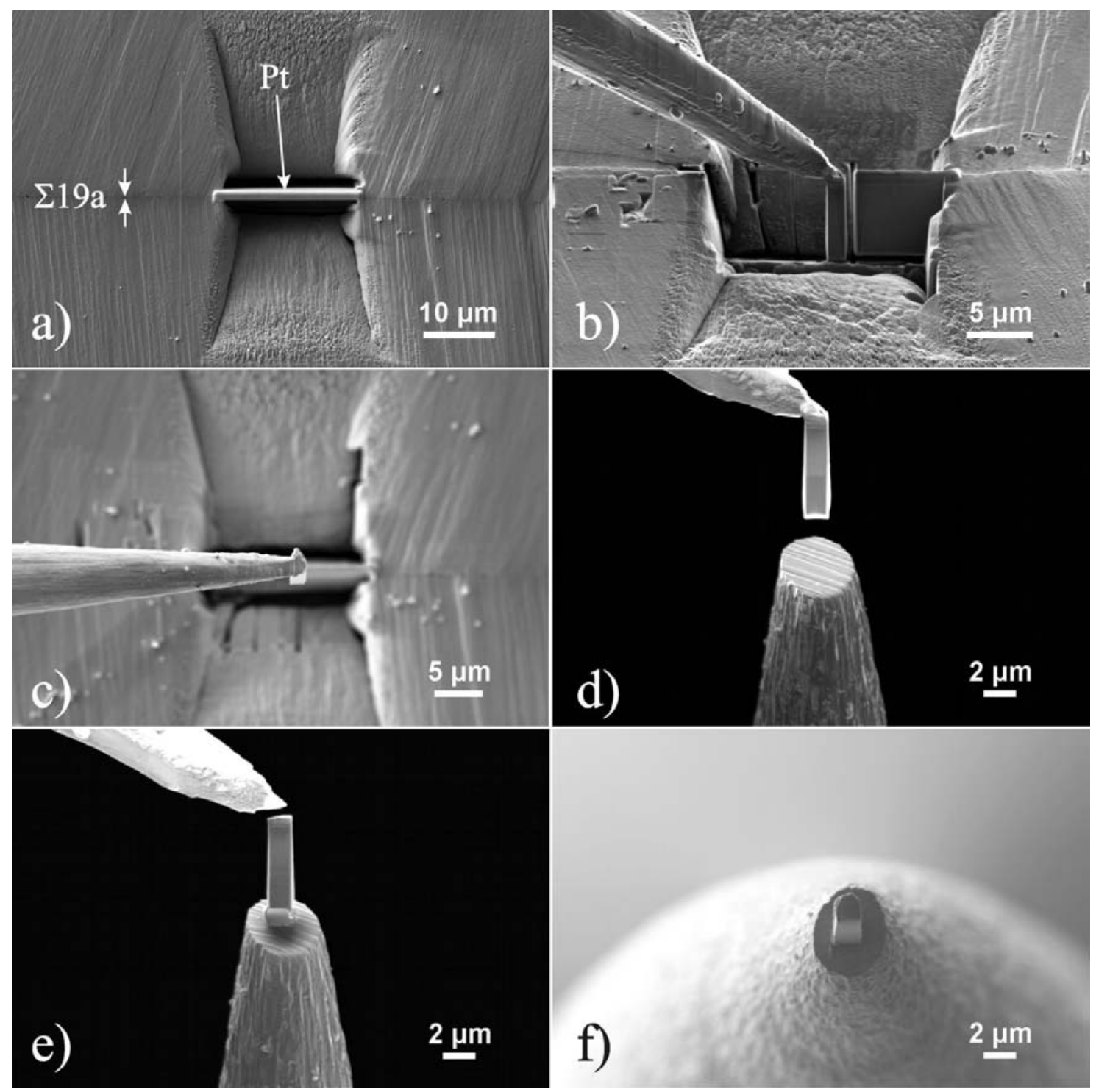

Abb. 3.10: Einzelne Schritte der ,Lift-Out“-Präparation im FIB; a) Schutz der KG durch Pt-Abscheidung, freilegen einer dicken Lamelle; b) Abtrennen eines Probenstückes mit enthaltener $K G$; c)-e) Transport zum Substrat, fixieren; f) fertiger Spitzenrohling

Der fertige Spitzenrohling in Abbildung 3.10f muss dann in weiteren Schritten zu einer geeigneten FIM/TAP-Spitze modelliert werden. Bei diesen Analysemethoden treten an der Spitze Zugspannungen von der Größenordnung $10^{10} \mathrm{~Pa}$ auf [GrOvENOR85], [MiLler96]. Um die Stabilität der Verankerung zu steigern wurde in einigen Fällen eine keilförmige Vertiefung in Probe und Substrat geschnitten (Abb. 3.11b), die dann mit Pt oder W aufgefüllt wurde (Abb. 3.11c). Dieser zusätzliche Anker steigerte die Stabilität 
leicht. Die für alle Abscheidungen verwendeten Gase waren $\mathrm{C}_{9} \mathrm{H}_{17} \mathrm{Pt}$ für die Pt- und $\mathrm{W}(\mathrm{CO})_{6}$ für die W-Abscheidung. Diese wurden durch ein Nadelventil ca. $350 \mu \mathrm{m}$ über der Probe freigesetzt. Mittels des Elektronen- oder $\mathrm{Ga}^{+}$-Ionenstrahls können dann die jeweiligen Metallatome vom Restmolekül abgetrennt werden. Die Parameter der Präparation finden sich in Tabelle 3.1.

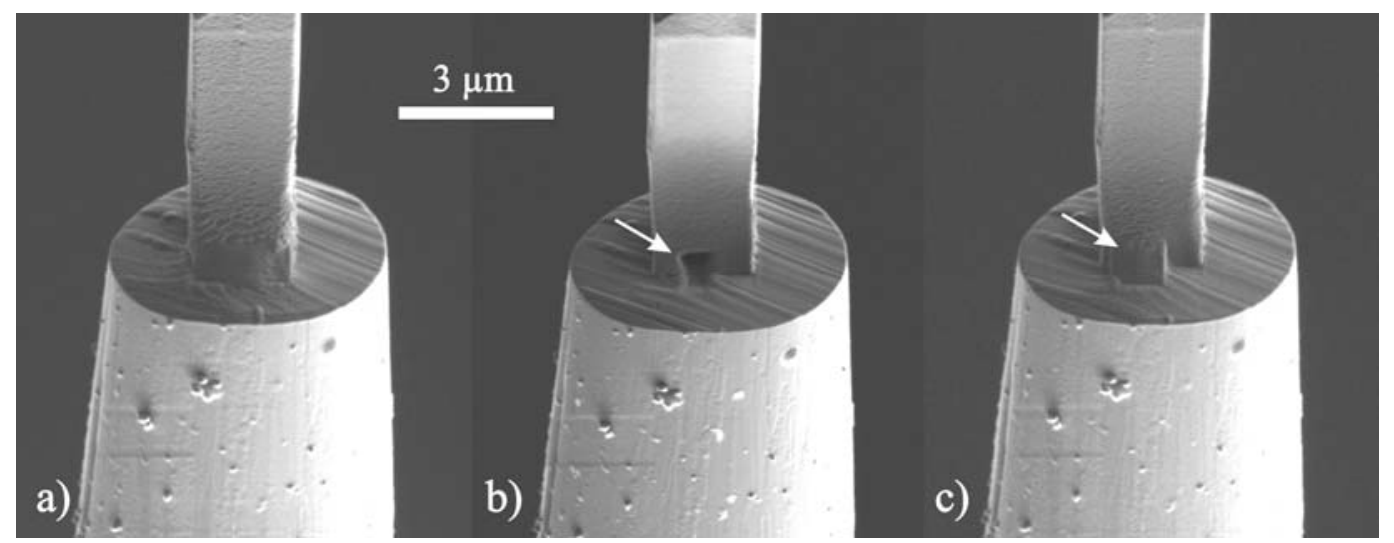

Abb. 3.11: Verbesserung der Verankerung; a) Ausgangszustand mit einfacher Verankerung; b) keilförmiges Graben in Probe und Substrat; c) Auffüllen des Grabens mit Pt oder $W$

\begin{tabular}{|l|l|l|}
\hline \multicolumn{1}{|c|}{ Anwendung } & \multicolumn{1}{c|}{ Strahlstrom } & \multicolumn{1}{c|}{ Dauer } \\
\hline Graben $\left(45^{\circ}\right.$ Gefälle) & $10 \mathrm{nA}-500 \mathrm{pA}$ & $\sim 40 \mathrm{~min}$ (pro Graben) \\
\hline Nivellieren der KG & $500-200 \mathrm{pA}$ & $\sim 5 \mathrm{~min}$ \\
\hline Feinschnitt an d. Lamelle & $200 \mathrm{pA}$ & $\sim 1 \mathrm{~min}$ (pro Schnitt) \\
\hline Pt-Abscheidung & $50-200 \mathrm{pA}$ & $\sim 1 \mathrm{~min}$ (pro Abscheidung) \\
\hline FIB-Abbildung & $50 \mathrm{pA}$ & $\sim 1 \mathrm{Bild} / \mathrm{s}$ \\
\hline
\end{tabular}

Tab. 3.1: Parameter und Dauer der Arbeitsschritte am FIB; Ga+ Beschleunigungsspannung $30 \mathrm{kV}$ 


\subsection{Simulation der Feldverteilung an FIM/TAP Spitzen}

Scharfe Kanten in der Nähe der Spitze (z.B. Stufen am Schaft) können Artefakte im FIM-Bild und Positionierungsfehler in TAP-Messungen hervorrufen. Dies ist offensichtlich, da die Spitze nicht nur die Probe, sondern auch das abbildende „Linsensystem“ der Meßmethode selbst ist. Bei der in dieser Arbeit vorgestellten Präparationsmethode mittels FIB wurden, bedingt durch unterschiedliche Herstellungsprozesse, Proben verschiedener Geometrien hergestellt und mit FIM/TAP untersucht. Es wurden häufig Artefakte in der Abbildung beobachtet, die im Folgenden erklärt werden sollen. Dazu wurden die exakte Potentiallandschaft in der Umgebung typischer FIB-Probengeometrien und die zugehörigen Ionentrajektorien mit dem Programm SIMION V7 [SIMION] durch lösen der Laplace-Gleichungen berechnet. Bei den ersten hergestellten Proben wurden die W-Substrate so abgeschnitten, dass ein Plateau entstand, welches eine wesentlich größere Grundfläche als das bikristalline Probenstück hatte. Es verblieb nach dem Modellieren der Spitze eine Stufe zum Substrat, deren störender Einfluss zunächst unterschätzt wurde. Die Abbildung 3.11 zeigt eine solche Probe und ihr zugehöriges FIM-Bild.
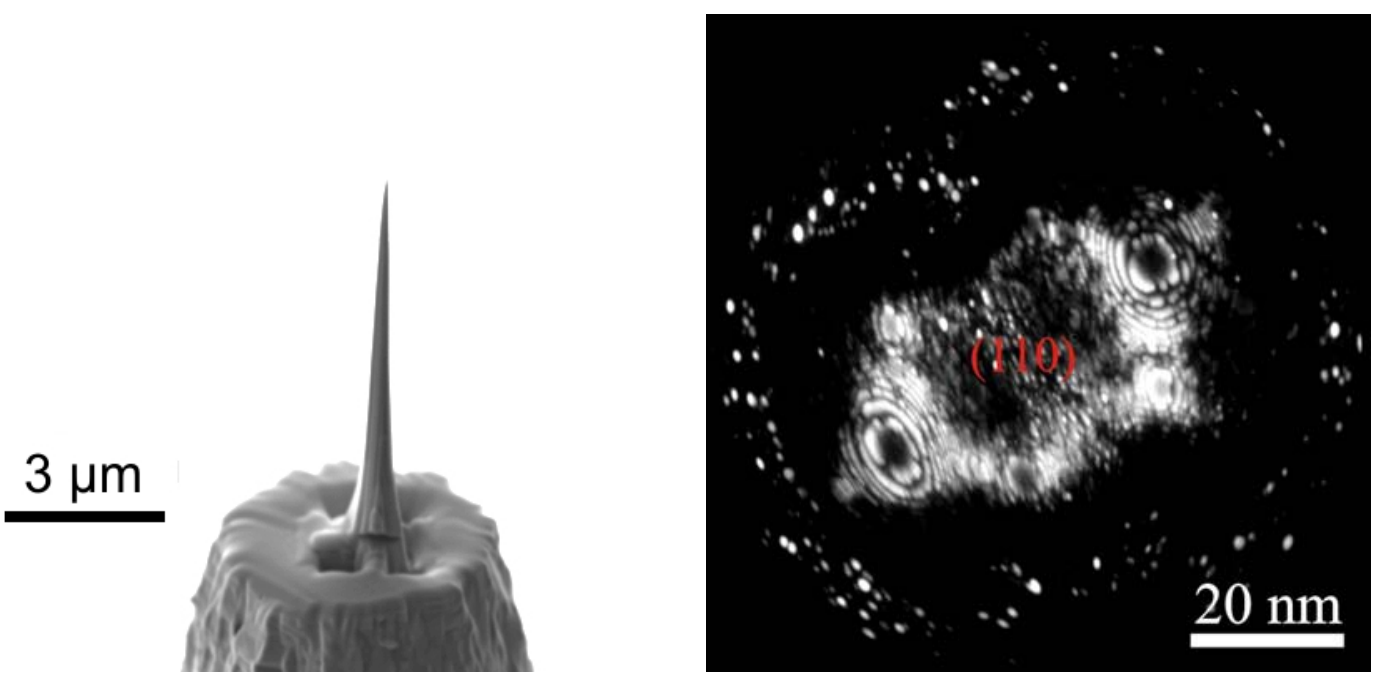

Abb. 3.12: Rechts: Bikristalline Probenspitze mit Stufe zum W-Substrat; an der Korngrenze befinden sich zwei Lunker; links: zugehöriges FIM-Bild mit äußerem, diffusem Saum 
Das FIM-Bild spiegelt die erwartete $<110>$-Richtung des $\mathrm{Cu}$-Bi-Bikristalls parallel zur Spitzenachse wider. In den Randbereichen wird nur ein diffuser Saum von Punkten abgebildet, wohingegen der Apexbereich mit atomarer Auflösung dargestellt wird. Alle Proben dieser Geometrie wiesen eine solche Einschränkung des beobachtbaren Bildbereiches auf. Auch in TAP-Analysen ist es bei kleinen Verkippungen der Spitze nicht mehr möglich, Atome in ausreichender Anzahl feldzuverdampfen. Beim Schneiden der Spitzen verbleiben am Übergang der Probe auf das Substrat häufig kleine Nebenspitzen übrig, die, wie die Stufe zum Substrat selbst, einen negativen Einfluss auf den Feldverlauf an der Spitze haben können. Die Abbildung 3.13a zeigt das elektrische Feld um eine ideale, mit $+10 \mathrm{kV}$ geladene Spitze, wobei die Wände der Simulationszelle auf Erdpotential sind. Der direkte Vergleich mit einer Spitze mit Stufe zum Substrat (Abb. 3.13b) zeigt eine deutliche Verzerrung der Potentiallinien. Zusätzlich wurden einige Trajektorien von ionisierten Spitzen- bzw. Bildgasatomen am Spitzenapex und an der Stufe zum W-Substrat berechnet. Ein Überlapp findet bereits etwa $50 \mu m$ entfernt von der Spitze statt. Da die typischen Abstände zwischen Spitze und FIM-Bildschrim bzw. TAP-Detektor zwischen 0,05-0,5 $\mathrm{m}$ liegen, sollten also solche Spitzengeometrien vermieden werden, um den in Abbildung 3.12 dargestellten diffusen Saum zu eliminieren.
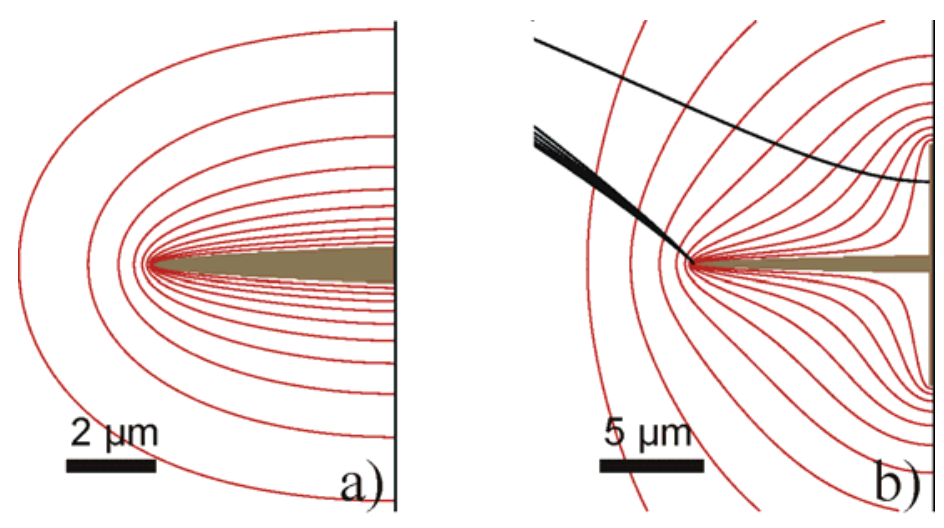

Abb. 3.13: a) elektrisches Potential in der Umgebung einer idealen FIM/TAP-Spitze; b) es treten deutliche Verzerrungen des Feldes durch die Stufe am Übergang auf das Substrat auf; Die Spannung an der Spitze wurde mit $U=10 \mathrm{kV}$ simuliert, Abstand der Equipotentilallinien ist $1000 \mathrm{~V}$; Krümmungsradius der Spitze ist $30 \mathrm{~nm}$. 
Insbesondere wird bei kreisender Schnittführung (siehe Abschnitt 3.4.1) mit variablen Radien entlang der zu modellierenden Spitzenachse oft eine große Anzahl von „Nebenspitzen“ erzeugt (Abb. 3.14).

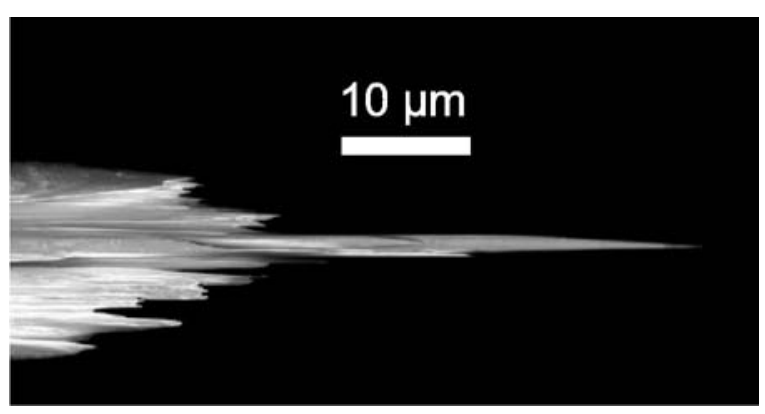

Abb. 3.14: Hohe Anzahl von Nebenspitzen nach einer kreisenden Schnittführung entlang der Achse der Spitze

Simulationen zeigen, dass diese die effektive Feldstärke am Apex der Spitze stark absenken. Es wurde hierfür eine zylindersymmetrische Form gewählt, um den Rechenaufwand der aufwendigen Simulation zu begrenzen. Dabei werden die einzelnen Nebenspitzen als Nebenspitzenring um die Hauptspitze modelliert (Abb. 3.15).

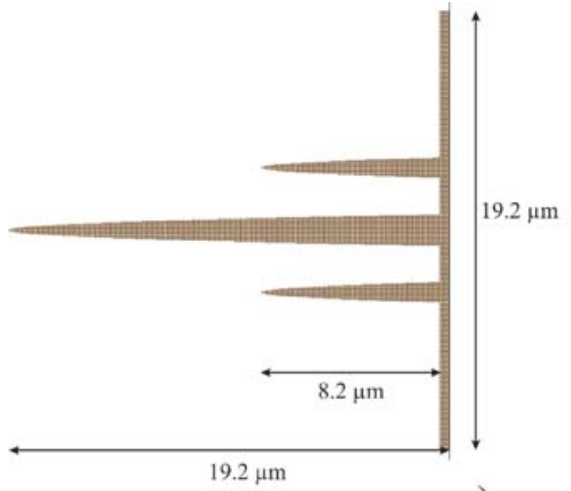

a)

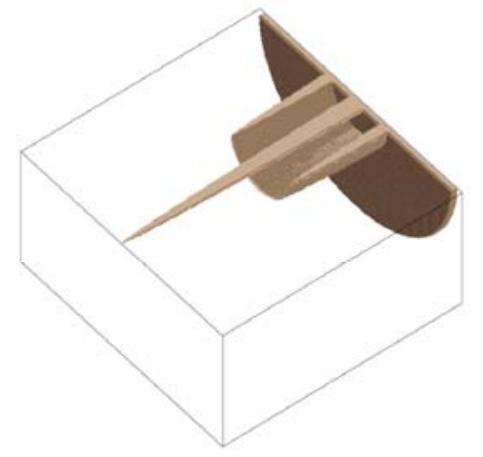

b)

Abb. 3.15: Modellierung einer zylindersymmetrischen Anordnung von Nebenspitzen zur Simulation der Potentiallandschaft; Hauptspitze und Nebenspitzenring haben einen Krümmungsradius von $30 \mathrm{~nm}$; Modell mit Abmessungen links, 3D Modell rechts

Sowohl der Krümmungsradius der Hauptspitze, wie auch des Nebenspitzenrings, wurde mit $30 \mathrm{~nm}$ gleich groß gewählt. 


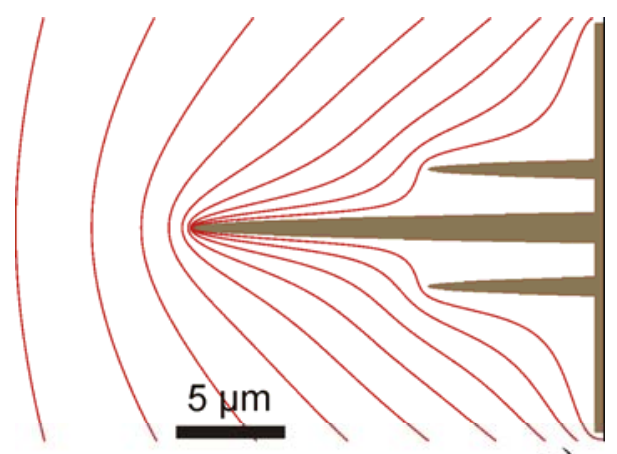

a)

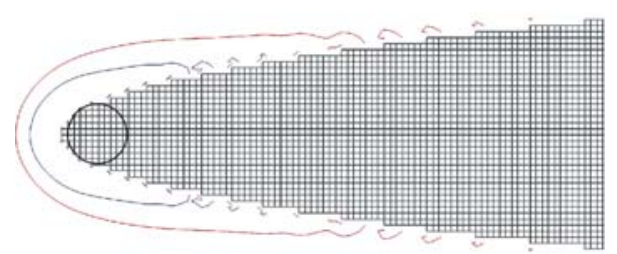

b)

Abb. 3.16: a) elektrisches Potential in der Umgebung einer Spitze mit Nebenspitzenring und Stufe zum W-Substrat; $U=10 \mathrm{kV}$, Abstand der Equipotentilallinien ist $1000 \mathrm{~V}$; b) direkter Vergleich der Potentiallandschaft einer Spitze mit (blau) und ohne Nebenspitzenring (rot); die Equipotentiallinien definieren jeweils zur Spitze hin die Bereiche mit Feldstärken $>30 \mathrm{~V} / \mathrm{nm}$; eingezeichneter Krümmungsradius $30 \mathrm{~nm}$

Die Potentiallandschaft einer solchen mit $+10 \mathrm{kV}$ geladenen Spitze wurde zunächst simuliert (Abb. 3.16a). Wiederum treten im Vergleich zur freistehenden Spitze deutliche Verzerrungen auf. Zusätzlich kann nachgewiesen werden, dass die Feldstärken von bis zu $30 \mathrm{~V} / \mathrm{nm}$ am Ort des Nebenspitzenrings bereits ausreichend für eine Feldverdampfung von Atomen oder eine Bildgasionisation sein können ${ }^{8}$. Dies verringert das effektive Feld am Apex der Hauptspitze, wie ein direkter Vergleich zwischen einer allein stehenden Spitze und derselben Spitze mit Nebenspitzenring zeigt (Abb. 3.16b). Der bei der FIM-Analyse erfassbare Bereich überstreicht typischerweise einen Winkel von $120^{\circ}$ über die Kugelkappe der Spitze. Die Bereiche mit Feldstärken $>30 \mathrm{~V} / \mathrm{nm}$ entsprechen in etwa der Feldverdampfungsfeldstärke von Neon und Kupfer und zeigen somit einen Trend zur Einengung des mit FIM/TAP analysierbaren Bereiches einer solchen Spitze. Es ist zu erwarten, dass dieser Trend durch diskrete Nebenspitzen statt des simulierten Nebenspitzenrings deutlich verstärkt wird.

\footnotetext{
${ }^{8}$ Kupfer hat eine Feldverdampfungsfeldstärke von $30 \mathrm{~V} / \mathrm{nm}$, das Bildgas Neon $35 \mathrm{~V} / \mathrm{nm}$.
} 
Aus diesen Simulationen ergeben sich zwei wichtige Folgerungen. Zum einen sollte bei der Präparation von FIM/TAP-Spitzen eine Stufe zum W-Substrat vermieden werden, wenn die maximale Ausdehnung des analysierbaren Bereichs benötigt wird. Besonders Nebenspitzen, die bei der Wahl einer kreisenden Schnittführung entstehen können, zerstören die Abbildungsgüte der Hauptspitze vollständig.

\subsection{Zielpräparation wohldefinierter Korngrenzen}

Die weitere Präparation zur FIM/TAP-Spitze muss so durchgeführt werden, dass der Korngrenzverlauf zu jedem Zeitpunkt ersichtlich ist. Es bestehen grundsätzlich zwei Schnittstrategien. Die Schnitte können entweder senkrecht von oben oder seitlich an der Probe durchgeführt werden (Abb. 3.17).

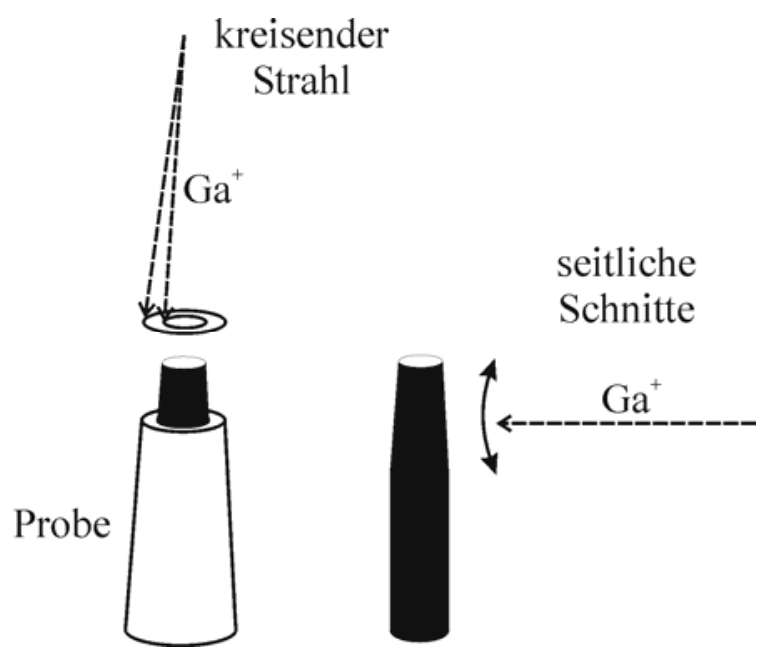

Abb. 3.17: Möglichkeiten der Schnittführung an einer FIM/TAP-Probe; links: Kreisender Ionenstrahl; rechts: Mehrere seitliche Schnitte, Probe wird in Einzelschritten um ihre Achse gedreht; die gleichzeitig zu bearbeitenden Bereiche sind schwarz gekennzeichnet.

Da ohne eine ständige optische Kontrolle keine Zielpräparation möglich ist, kommt nur eine seitliche Schnittführung mit ständigem Blick auf den Korngrenzverlauf in Frage. Wie Simulationen zeigten, ist diese Methode aufgrund des Entstehens von Stufen und Nebenspitzen bei kreisendem Ionenstrahl wesentlich vorteilhafter. Zusätzlich wird eine 
Implantation von $\mathrm{Ga}$ in das Probenmaterial bei der seitlichen Schnittführung größtenteils vermieden. Für sehr feine Schnitte wurde ausschließlich das durch $\mathrm{Ga}^{+}-$ Ionen erzeugte Sekundärelektronenbild zur Positionierung verwendet. Um die GaKontamination möglichst gering zu halten wurde der Strahlstrom auf $50 \mathrm{pA}$ reduziert. Mit geringsten Strahlströmen $(\leq 50 p A)$ wird die endgültige Form der Spitze definiert. Ein Krümmungsradius von $20-60 \mathrm{~nm}$ kann bei einem Schaftwinkel von $4-10^{\circ}$ problemlos erreicht werden. Es wurde zuvor mit Feldsimulationen gezeigt, dass der in FIM und TAP beobachtbare Bereich solcher Spitzen durch die erhebliche Stufe zum Substrat eingeschränkt wird. Diese wurde durch die Wahl eines kleineren Substratdurchmessers bei den folgenden Proben vermieden. Das überstehende Probenstück konnte somit zu einer Spitze modelliert werden und passgenau in das Substrat integriert werden. Die Abbildung 3.18 zeigt eine vollständige Präparationssequenz mit einem dünneren W-Substrat. Durch die zylindersymmetrische Geometrie bei einem Krümmungsradius unter $50 \mathrm{~nm}$ ist diese Spitze sehr gut als FIM/TAP-Probe geeignet.

(a)

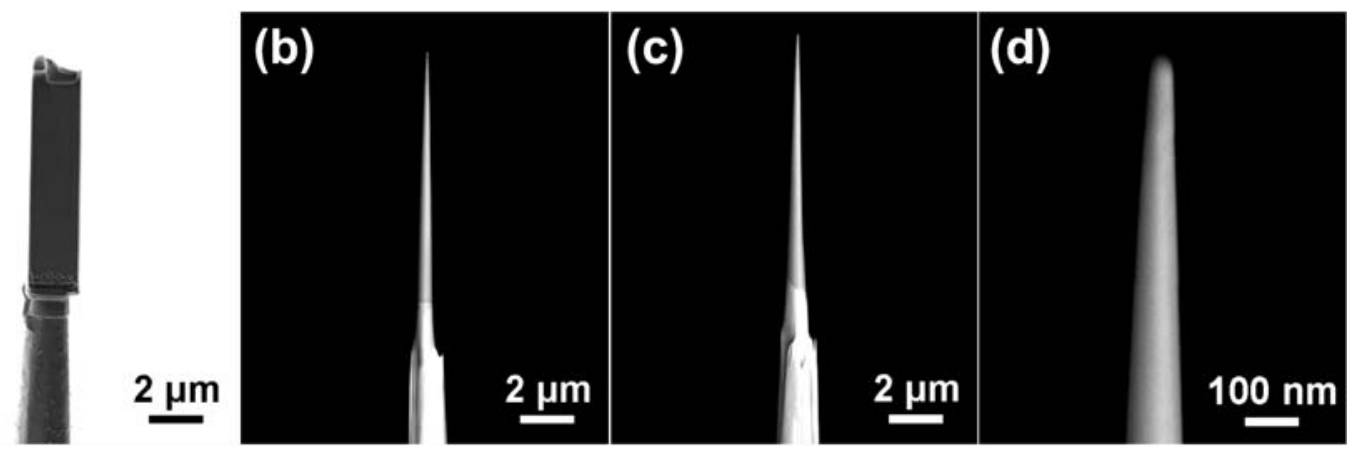

Abb. 3.18: Zielpräparation einer idealen FIM/TAP-Spitze auf einem dünnen W-Substrat; a) verankertes Probenstück; b), c) jeweils um $90^{\circ}$ gedrehte Ansicht der Spitze; d) Vergrößerung des Apexbereiches

Die vorgestellte Methode ist eine deutliche Erweiterung der von Miller et al. [MiLLER04] vorgeschlagenen Präparation von LEAP ${ }^{9}$-Proben, bei denen der Effekt überlappender Trajektorien durch eine lokale Extraktionselektrode am Ort des Spitzenapex reduziert wird. Solche Proben wären aufgrund der Feldverteilung in ihrer

\footnotetext{
${ }^{9}$ Local Electrode Atom Probe (LEAP)
} 
Umgebung untauglich für FIM/TAP-Analysen. Insbesondere die Präparation einer FIM/TAP-Spitze mit einer einzelnen wohldefinierten Korngrenze, ist nur unter ständiger Kontrolle des Korngrenzverlaufs möglich, wie sie erstmals nur mit der in dieser Arbeit entwickelten Methode möglich ist. 


\section{Ergebnisse}

Im Folgenden werden die Ergebnisse zur nanostrukturellen Entwicklung der untersuchten $\mathrm{Ag}-\mathrm{Ni}$ - und $\mathrm{Cu}-\mathrm{Bi}$ - Proben vorgestellt. Es werden zunächst die Ergebnisse der co-evaporierten und gesputterten Ag-Ni-Proben dargestellt. Im zweiten Teil werden die Ergebnisse an einzelnen $\Sigma 19 a$ Korngrenzen in Cu-Bi Bikristallen präsentiert. Die Facettierung dieser Korngrenze wurde im segregierten Zustand zusätzlich mittels hochauflösender Transmissionselektronenmikroskopie (HR-TEM) untersucht.

\subsection{Untersuchung der co-evaporierten Ag-Ni Proben}

\subsubsection{Nanostruktur des „as-prepared“-Zustandes}

Zur Analyse der zeitlichen Entwicklung des Gefüges wurden Auslagerungsexperimente an funkenerodierten Spitzenrohlingen und kumulative Auslagerungen an bereits gemessenen Spitzen durchgeführt ${ }^{10}$. Die Auslagerungszeiten betrugen zwischen 10 und 30 Minuten bei Temperaturen zwischen $200^{\circ} \mathrm{C}$ und $400^{\circ} \mathrm{C}$. Es wurden zwei Ag-NiProbenserien mit hohem und niedrigem Ni-Gehalt untersucht. Co-evaporierte und anschließend mit $1 \mathrm{GPa}$ kompaktierte Ag-Proben haben, im Vergleich zu kristallinem Material, eine um etwa $4 \%$ reduzierte Dichte [QIN95]. Dies erschwert die Analyse durch FIM/TAP erheblich, da die Proben an ausgedehnten Porositäten mechanisch versagen. Die Abbildung 4.1 zeigt eine typische Oberfläche einer thermisch nicht behandelten Probe. Man erkennt bis zu $5 \mu \mathrm{m}$ ausgedehnte Risse, die durch die hohe Verformung über den plastischen Bereich hinaus entstanden sind. Die Polfigur zeigt eine für alle Proben typische $<111>-$ Textur in Pressrichtung. Durch EDX-Linescans wurden die mittleren Ni-Gehalte der Probenserien $\mathrm{zu} 3,1(1,5)$ At.\% und

\footnotetext{
${ }^{10}$ Kleine Probenmengen (jeweils einige $10 \mathrm{mg}$ ) erforderten ein mehrmaliges Nachdünnen kumulativ ausgelagerter FIM/TAP-Spitzen.
} 
$14,3(1,3)$ At. $\%$ bestimmt. Im Folgenden werden die jeweiligen Proben daher als Proben mit hohem bzw. niedrigem Ni-Gehalt bezeichnet.
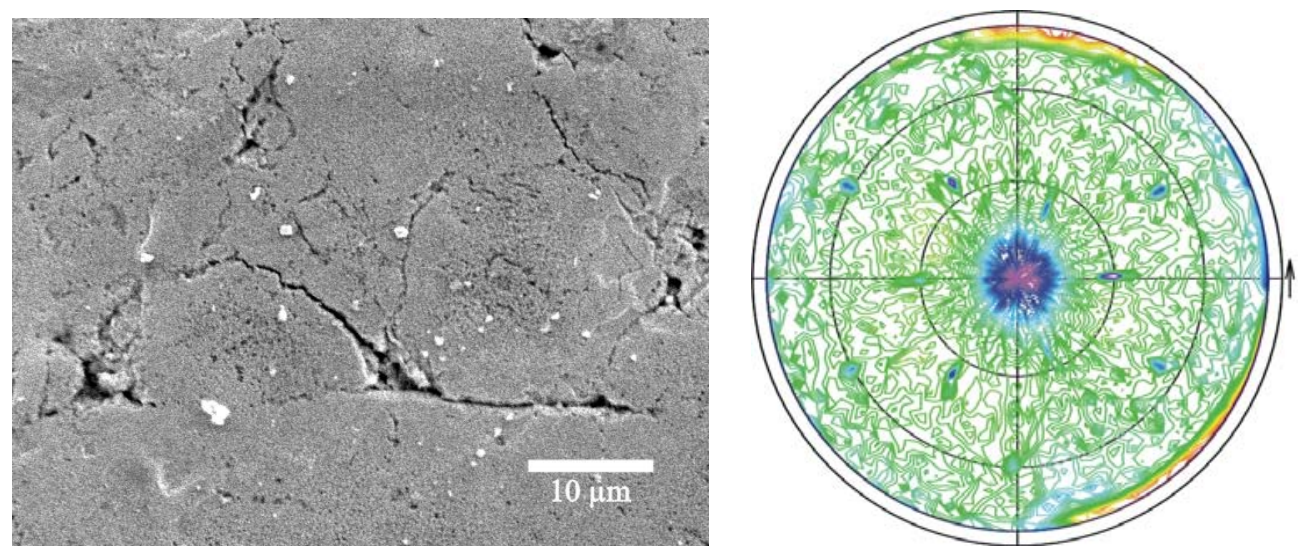

Abb. 4.1: Links: Typische Oberfläche einer co-evaporierten Ag-Ni-Probe; bis zu $5 \mu \mathrm{m}$ ausgedehnte Risse sind im Rasterelektronenmikroskop zu erkennen; rechts: $<111>-$ Textur dieser Proben (hohe Intensität violett, niedrige Intensität rot)

Die Ag-Ni-Proben mit hohem Ni-Gehalt zeigen im FIM-Bild einen deutlich zu erkennenden Kontrast zwischen hellen Ni-Körnern und der dunklen Ag-Matrix (Abb. 4.2). Dieser Kontrast ist aufgrund der stark unterschiedlichen Feldverdampfungsfeldstärken der beiden Materialien so ausgeprägt, dass in Anwesenheit der Ni-Körner keine Ag-Matrixatome sichtbar sind. Im hergestellten Zustand (,as-prepared“) sind bei hoher Vergrößerung einzelne Ni-Körner zu erkennen, die in einigen Fällen kettenartig aufgereiht sind. Aus den beobachteten Ringstrukturen der Ni-Körner kann eine amorphe, Ni-haltige Phase ausgeschlossen werden. Somit kann eine kristalline Struktur bereits im ,as-prepared“ Zustand nachgewiesen werden. Das FIM-Bild des gleichen Zustandes (Abb. 4.3) zeigt eine Übersicht der Körner bei kleinerer Vergrößerung. Aus den Bildern kann eine Ni-Korngrößenverteilung mit guter Statistik ausgezählt werden (Abb.4.4). Da keine ideal kugelförmigen Ni-Körner vorliegen, wurde jeweils über die Ausdehnungen in $x$ - und $y$-Richtung des Korns gemessen. Eine Vorzugsrichtung ist in diesem Zustand nicht erkennbar. Die aufgetragenen Werte sind das geometrische Mittel dieser Werte. Die mittlere Korngröße beträgt $3 \pm 2 \mathrm{~nm}$. Auffällig sind viele besonders kleine Körner, die nur aus wenigen NiAtomen bestehen. 


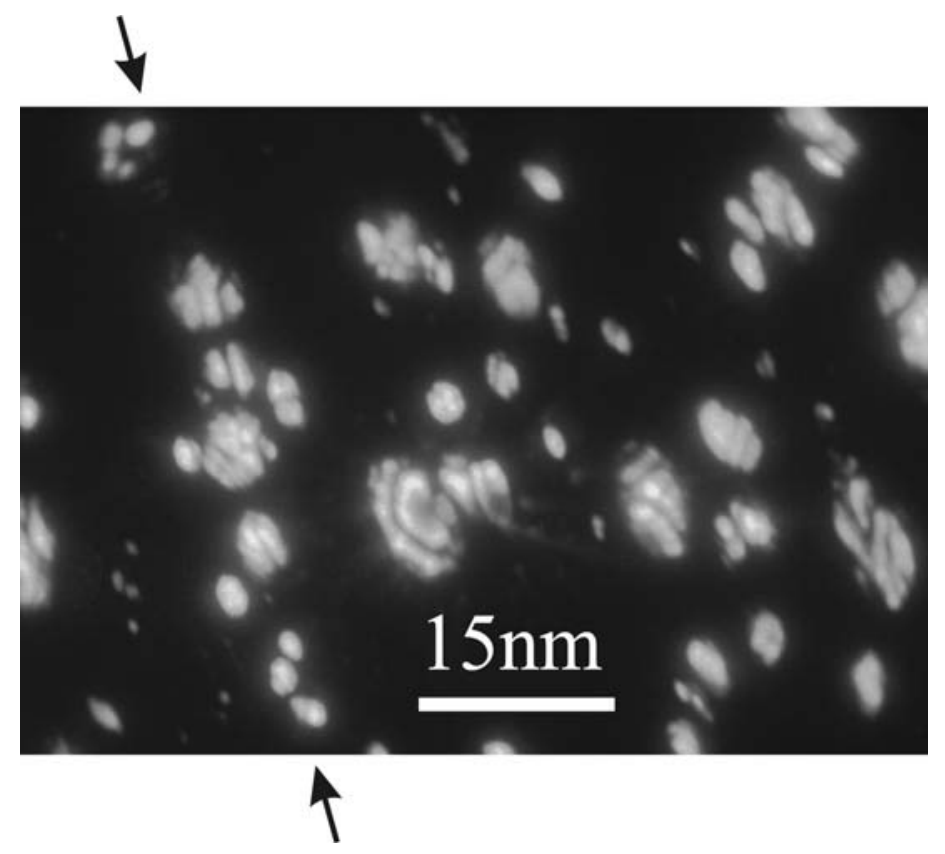

Abb. 4.2: FIM-Bild einer Ag-Ni Probe mit hohem Ni-Gehalt; kettenartig aufgereihte NiKörner sind mit Pfeilen gekennzeichnet.

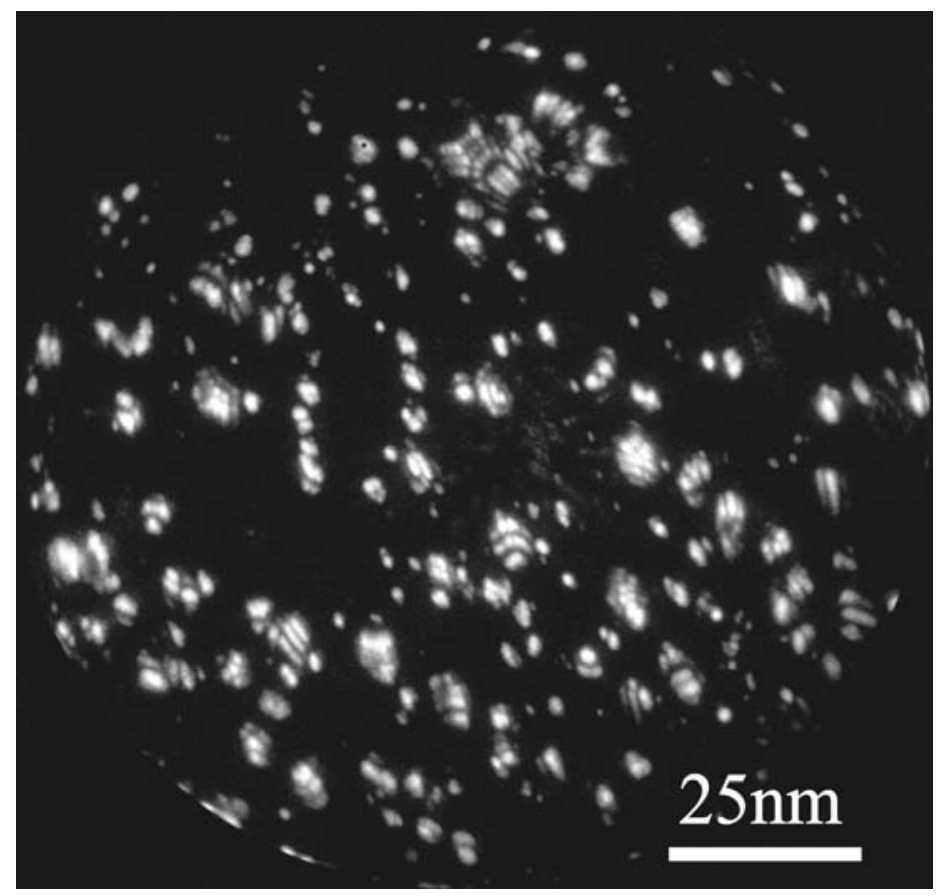

Abb. 4.3: FIM-Übersichtsbild des gleichen Zustandes wie Abb. 4.2 


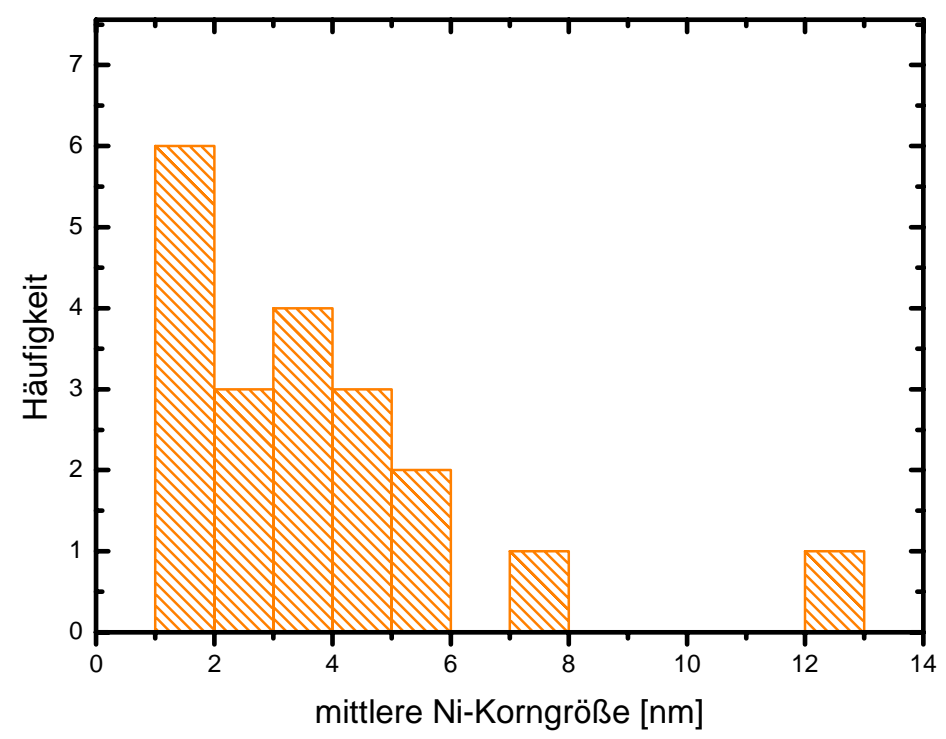

Abb. 4.4: Ni-Korngrößenverteilung der Ag-Ni-Probe mit hohem Ni-Gehalt im „asprepared"-Zustand

Durch Zählen aller detektierten Atome ergibt sich aus TAP-Messungen ein mittlerer NiGehalt von 7,6 At. \%. Aus den Flugzeiten kann das Masse-zu-Ladungsverhältnis für alle erfassten Ionen aufgetragen werden. Die Massenspektren aller co-evaporierten Proben zeigen typischerweise Verunreinigungen kleiner $1000 \mathrm{ppm} \mathrm{At. \% .} \mathrm{Oft} \mathrm{werden} \mathrm{jedoch}$ Ag-Zweifachhydride beider Ag-Isotope beobachtet, was durch eine Überlagerung zu drei ausgeprägten Peaks bei 107, 109 und 111 amu führt (Abb. 4.5). Da bekannt ist, dass Ag keine stabilen Hydride bildet, ist eine gemeinsame Feldverdampfung von AgAtomen der Spitze und $\mathrm{H}_{2}$-Molekülen aus dem Restgas der UHV-Kammer eine nahe liegende Erklärung für das beobachtete Phänomen. 


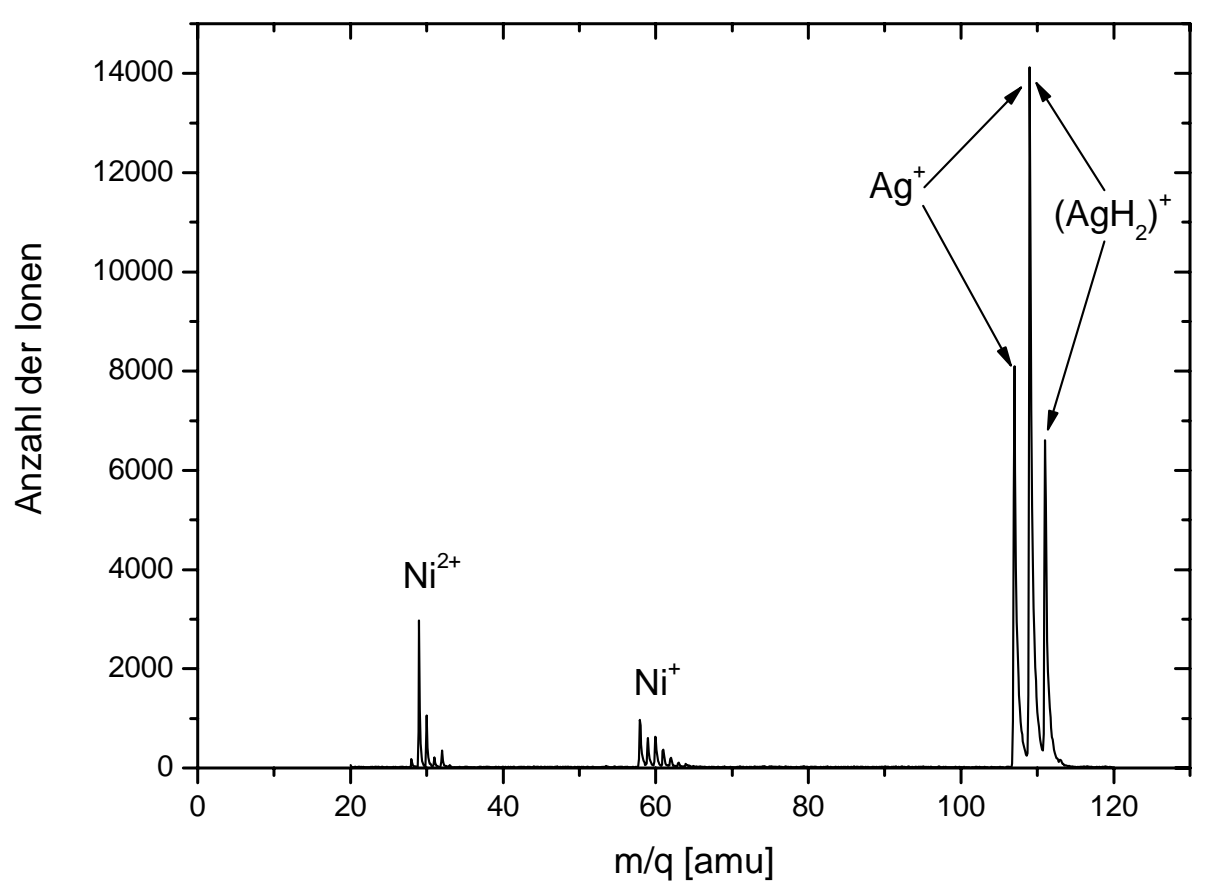

Abb. 4.5: Massenspektrum einer Ag-Ni Probe mit hohem Ni-Gehalt

Die Ag-Kornstruktur kann aufgrund des starken Kontrastunterschiedes nicht mit der Feld-Ionen-Mikroskopie ermittelt werden. Unter günstigen Messbedingungen kann diese jedoch in TAP-Analysen direkt sichtbar gemacht werden. In einigen Ag-Körnern sind Atomlagen zu erkennen (Abb. 4.6b) ${ }^{11}$. Die Korngrenzen der Matrix werden als Flächen mit abweichender, zumeist reduzierter, Dichte rekonstruiert. Die Kornstruktur kann wie in Abbildung. 4.6c ersichtlich schematisch dargestellt werden. Die Körner können als plattenförmig bezeichnet werden, wobei die Plattendicke senkrecht zur Messrichtung stets die geringste Ausdehnung hat. Die mittleren Kornausdehnungen betragen für die Länge und Dicke der Körner > $30 \mathrm{~nm}$ und 2 - $7 \mathrm{~nm}$. Ni-Anreicherungen finden sich sowohl zwischen den Ag-Körnern wie auch innerhalb einiger Ag-Körner (Abb. 4.6a).

\footnotetext{
${ }^{11}$ Die Auflösbarkeit der Atomlagen hängt stark von der jeweiligen Kornorientierung ab (siehe Abschnitt 2.1.2).
} 


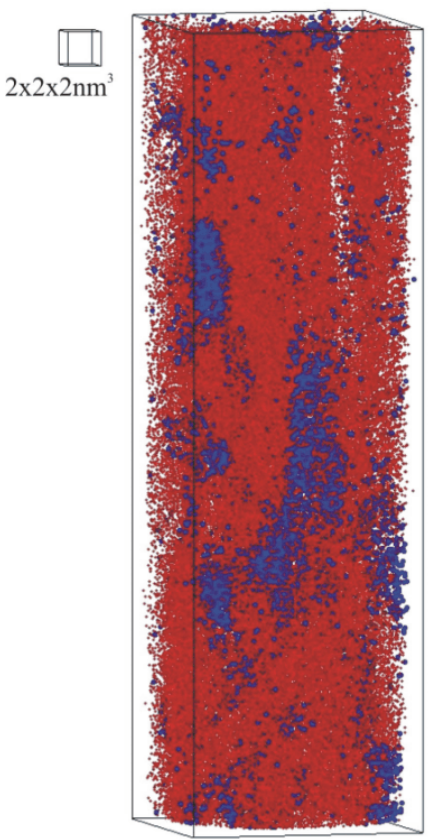

a)

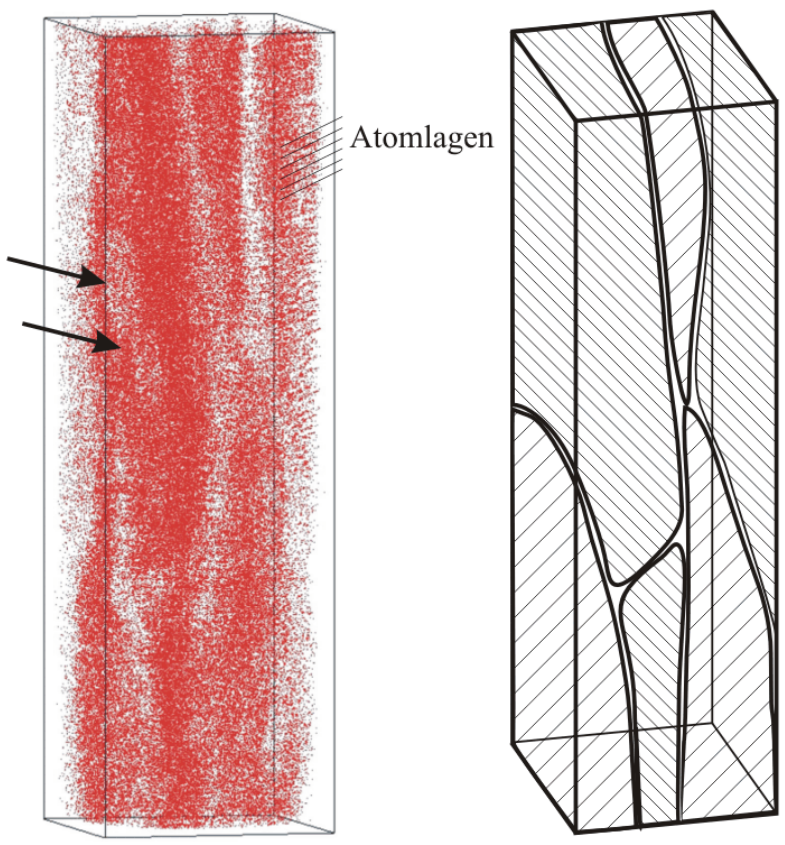

b)

c)

Abb. 4.6: TAP-Messung einer Probe mit hohem Ni-Gehalt; a) Darstellung aller Atome; b) nur Ag-Atome; Positionen von Ni-Einschlüssen in einem Ag-Korn sind mit Pfeilen gekennzeichnet; c) schematische Anordnung der Körner im analysierten Volumen

Ein wesentliches Problem ergibt sich bei der Messung durch mechanische Instabilitäten der Spitze. Diese entstehen durch kleine Porositäten, die in Abständen von $50-60 \mathrm{~nm}$ auftreten. Die Abmessungen dieser Defekte können nur geschätzt werden, da die Messmethode gerade an ausgedehnten Poren versagt. Typisch für das Versagen sind sog. Mikroabrisse, die mit einem kleinen Spannungssprung in der Messung und einem Versatz der Nanostruktur im rekonstruierten Volumen verbunden sind. Die Geometrie der Spitze bleibt dabei im Wesentlichen erhalten. Aus einem Vergleich der Kornstrukturen vor und nach einem Mikroabriss der Spitze ergibt sich eine mittlere Porengröße von ca. $10 \mathrm{~nm}$ in Analyserichtung (Abb. 4.7). Die Korngrenze wird in ihrem Verlauf über den Abriss geometrisch fortgesetzt. Am Ort der Deckungsgleichheit beider Teilstücke kann eine Abrisstiefe (Porengröße) geschätzt werden. Die Porengröße stellt eine untere Abschätzung der Porengröße dar. 


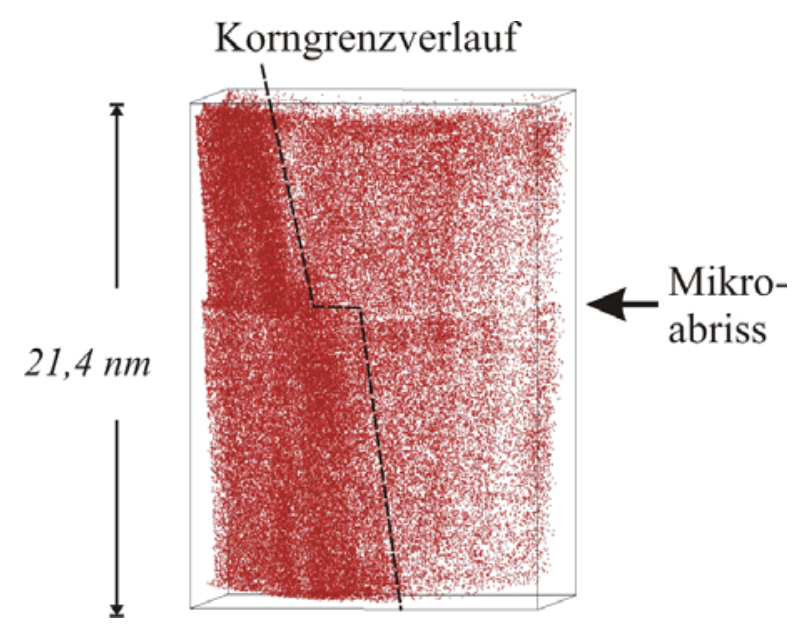

Abb. 4.7: Versatz des Korngrenzenverlaufs an einem Mikroabriss; eine typische Porengröße von ca. $10 \mathrm{~nm}$ in Analyserichtung wird für die Proben mit hohem Ni-Gehalt geschätzt.

Für die unbehandelten Ag-Ni-Proben mit niedrigem Ni-Gehalt zeigt das FIM-Bild nur vereinzelt Ni-Körner (Abb. 4.8). Ihre Anzahldichte ist so gering, dass in Abwesenheit des starken Phasenkontrastes weite Teile der Ag-Matrix atomar aufgelöst werden können. Die Ni-Anreicherungen werden bei den beobachteten Ausdehnungen von weniger als $1 \mathrm{~nm}$ oft nur als helle Punkte dargestellt. Etwas größere Ni-Anreicherungen mit Ausdehnungen bis $3 \mathrm{~nm}$ zeigen Ansätze einer Ringstruktur, weswegen bei diesen von Ni-Körnern mit einer kristallinen Struktur ausgegangen werden kann. Die Darstellung der Verteilung der Ni-Atome durch TAP-Analysen zeigt keine Ni-Körner (Abb. 4.9a/b). Dies steht, aufgrund des wesentlich kleineren Messvolumens des TAP, in guter Übereinstimmung mit dem Befund vereinzelter Ni-Körner im FIM-Bild. 


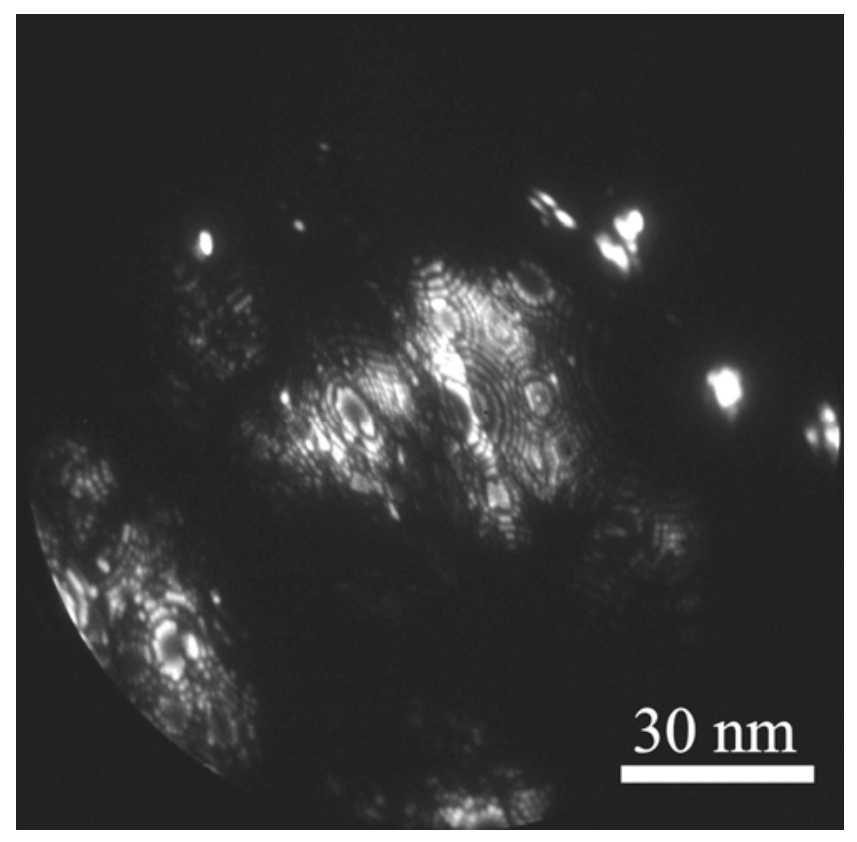

Abb. 4.8: FIM-Bild einer unbehandelten Ag-Ni Probe mit niedrigem Ni-Gehalt; die AgMatrix wird atomar aufgelöst.

Die Kornstrukturen sind, wie im Fall der Proben mit hohem Ni-Gehalt, plattenförmig und überschreiten in ihrer Länge das analysierbare TAP-Volumen. Da nie zugleich Anfang und Ende eines Ag-Korns in Analyserichtung erfasst wurden, kann die Korngröße in der Plattenebene mit $>70 \mathrm{~nm}$ angegeben werden. Die Plattendicke variiert zwischen 3-5 $\mathrm{nm}$. Eine schematische Darstellung der beobachteten Kornstruktur wird in Abbildung 4.9c gegeben. Obwohl praktisch keine Löslichkeit von $\mathrm{Ni}$ in Ag bei der Kondensationstemperatur von $77 \mathrm{~K}$ existiert [MASSALSKI90], zeigen TAP-Analysen Ag-Körner mit Ni-Gehalten bis 1,5 At.\% (Abb. 4.10). Um der Fragestellung nach $\mathrm{zu}$ gehen, ob die vorliegende Ni-Verteilung innerhalb des Messvolumens statistisch ist oder nicht, wurde ein $\chi^{2}$-Test durchgeführt. 


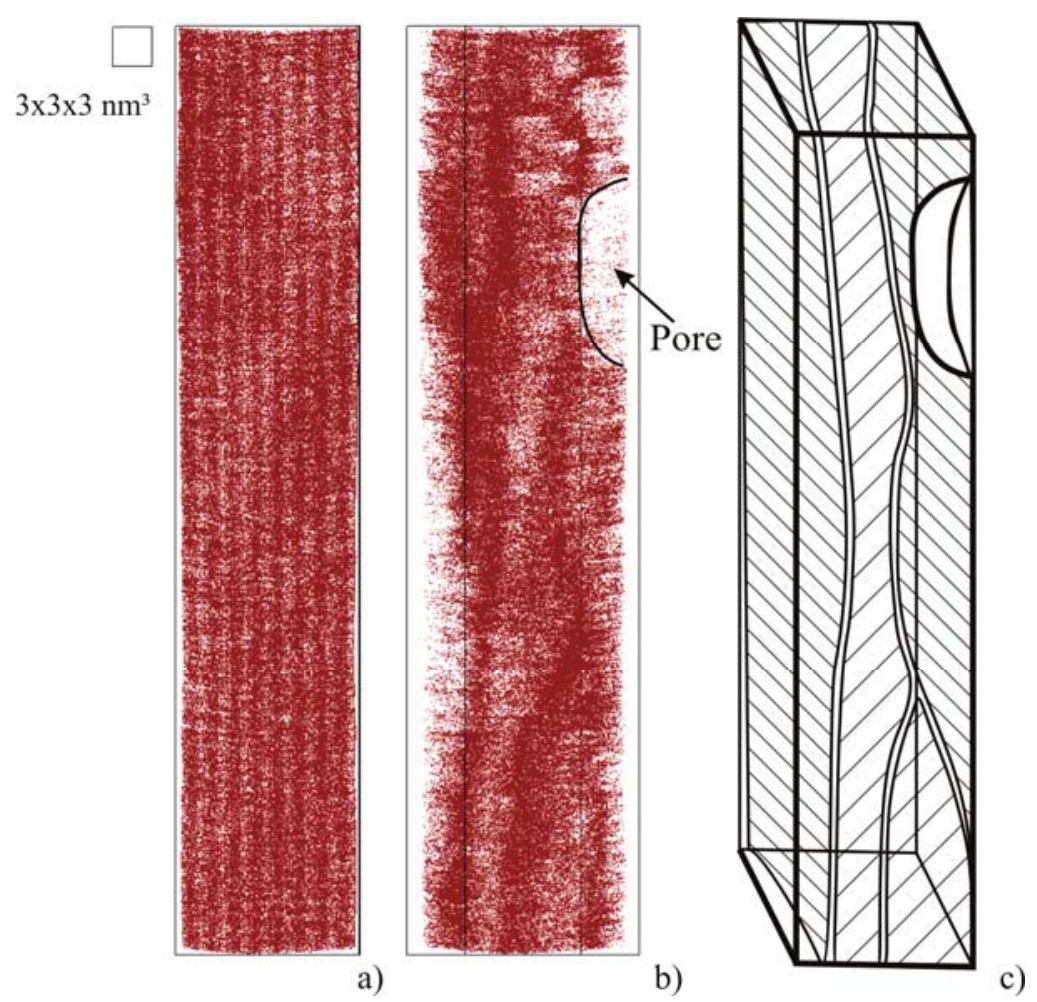

Abb. 4.9: Verteilung der Ag-Atome einer Probe mit niedrigem Ni-Gehalt; a) Blick entlang der angenommenen Kompaktierungsrichtung; b) Volumen um $90^{\circ}$ gedreht; eine ca. $10 \mathrm{~nm}$ ausgedehnte Pore in Ag-Korn ist zu erkennen; c) Schematische Darstellung der Ag-Kornstruktur

Um die Hypothese einer homogenen Ni-Verteilung zu überprüfen wurde die gemessene Konzentrationsverteilung mit der zugehörigen Binomialverteilung verglichen (Abb. 4.11). Es zeigt sich eine sehr große Abweichung des gemäß (2.7) berechneten $\chi^{2}$-Wertes von 2954 vom Vergleichswert 14,1, der einem Signifikanzniveau von $95 \%$ entspricht. Folglich ist die gemessene Ni-Verteilung als inhomogen zu betrachten.

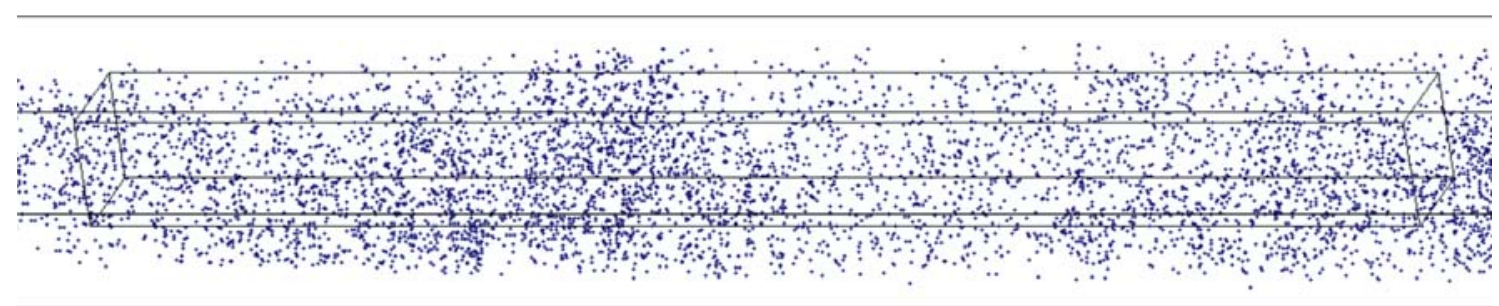

Abb. 4.10: Verteilung der Ni-Atome innerhalb eines rekonstruierten Ag-Korns; äußere Abmessungen 7,7 × 7,7 $\times 9,4 \mathrm{~nm}^{3}$; die Bestimmung des $\chi^{2}$-Wertes wurde im inneren Quader durchgeführt; reine Ni-Körner sind nicht vorhanden. 


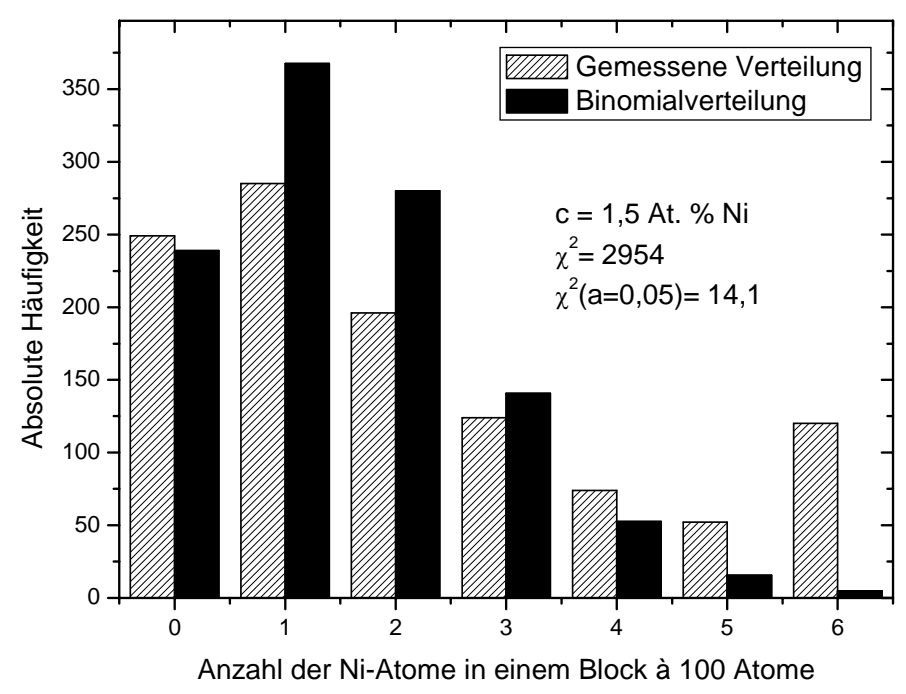

Abb. 4.11: Gemessene Ni-Konzentrationsverteilung in einer Probe mit niedrigem NiGehalt und zugehörige Binomialverteilungen

\subsubsection{Ni-Verteilung in wärmebehandelten Proben}

Bereits nach einer Auslagerung von $10 \mathrm{~min}$ bei $200{ }^{\circ} \mathrm{C}$ ist eine deutliche Zunahme der Ni-Korngröße in den Proben mit hohem Ni-Gehalt zu beobachten. Aufgrund des lokalen Vergrößerungseffekts wird eine Visualisierung der Ni-Körner durch deren zugehörige silberfreie Bereiche bevorzugt. Die Ni-Körner sind dadurch als offensichtliche Löcher in der Matrix zu erkennen (Abb.4.12). Bei etwa gleicher Standardabweichung haben sich die beobachteten Korngrößen etwa verdoppelt. Einige Körner sind, im Gegensatz zum Ausgangszustand, senkrecht zur Messrichtung elongiert. Interessanterweise werden viele $\mathrm{Ni}$-Körner beobachtet, die eng benachbart sind und durch wenige monolagenbreite Stege aus reinem Ag voneinander getrennt sind (Abb. 4.12 Pfeile). Die glatte, lamellare Korngrenzstruktur des unbehandelten Zustandes wird nicht mehr beobachtet. Stattdessen liegt eine wellenförmige Struktur vor, die von Ni-Körnern begrenzt wird. Da das TAP-Analysevolumen nur einen beschränkten Sichtbereich in lateraler Richtung bietet, können diese zur Messrichtung verkippten Korngrenzen nicht mehr ihrer Länge nach erfasst werden. 

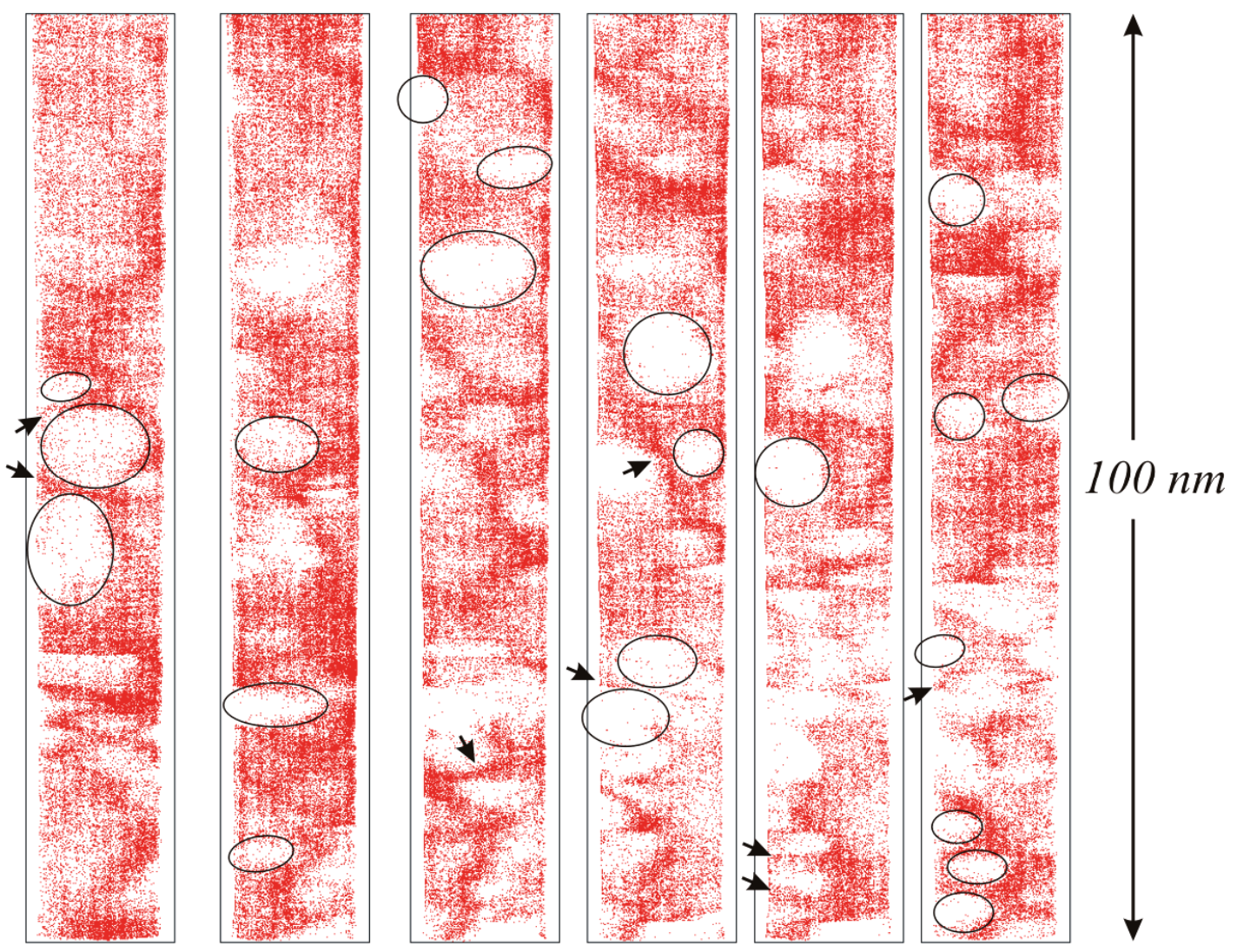

Abb. 4.12: Verteilung der Ag-Atome innerhalb einer Probe mit hohem Ni-Gehalt nach 10 min bei $200^{\circ} \mathrm{C}$; das Volumen wurde von links nach rechts sukzessive in Scheiben der Dicke 2 nm geschnitten; Ni-Körner wurden an ihrem größten Durchmesser markiert

Eine Segregation von Ni-Atomen in die Ag-Korngrenzen wird nicht beobachtet.

Nach einer Auslagerung von $30 \mathrm{~min}$ bei $400^{\circ} \mathrm{C}$ zeigen TAP-Anaylsen eine starke Tendenz zur Bildung von plattenförmigen Ni-Körnern, wobei die Plattennormale parallel zur Messrichtung orientiert ist (Abb. 4.13a/b). In diesem Zustand wurden keine sphärischen Ni-Anreicherungen mehr detektiert. Mehrere Analysen bestätigen, dass sich die Ni-Platten bevorzugt in Gruppen formieren und dabei einen gegenseitigen Abstand von wenigen Nanometern in der Ag-Matrix einnehmen. Die Platten überschreiten in ihrer Ausdehnung den analysierbaren Probenbereich in lateraler Richtung deutlich. Eine Konzentrationsanalyse wurde entlang des in Abbildung 4.13c eingezeichneten Zylinders durchgeführt. Der Verlauf wurde in Abbildung 4.14 graphisch mit den jeweiligen Ausdehnungen dargestellt. 


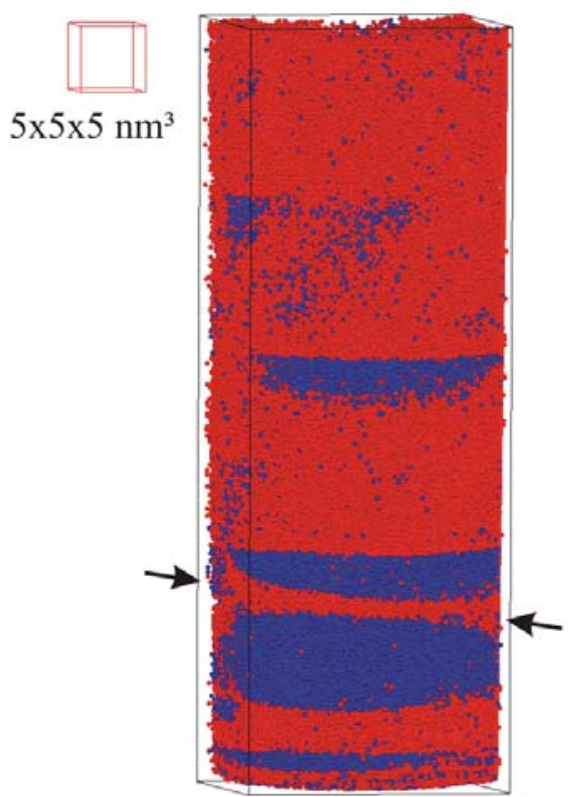

a)

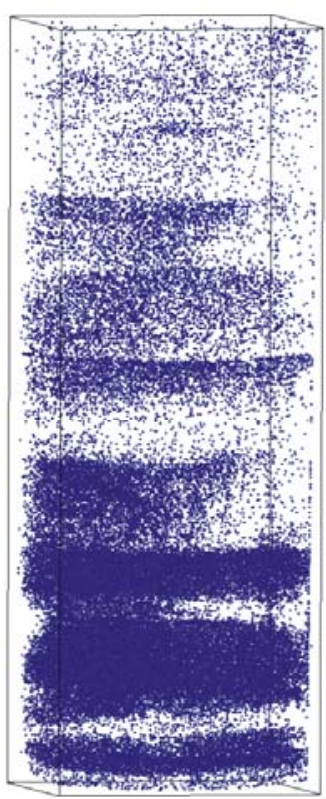

b)

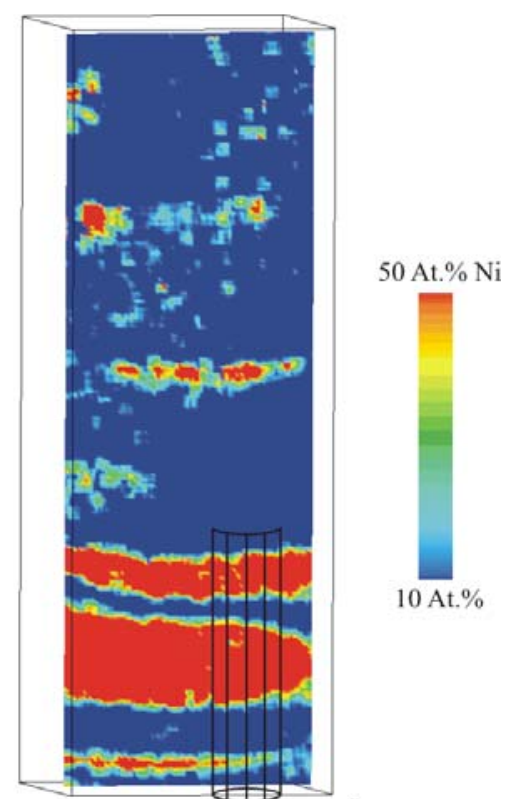

c)

Abb. 4.13: TAP-Analyse einer Probe mit hohem Ni-Gehalt; Darstellung der a) Ag-und $\mathrm{Ni}$-Atome; b) Ni-Atome; c) Konzentrationsverteilung auf einer Ebene in Falschfarben und Analysezylinder

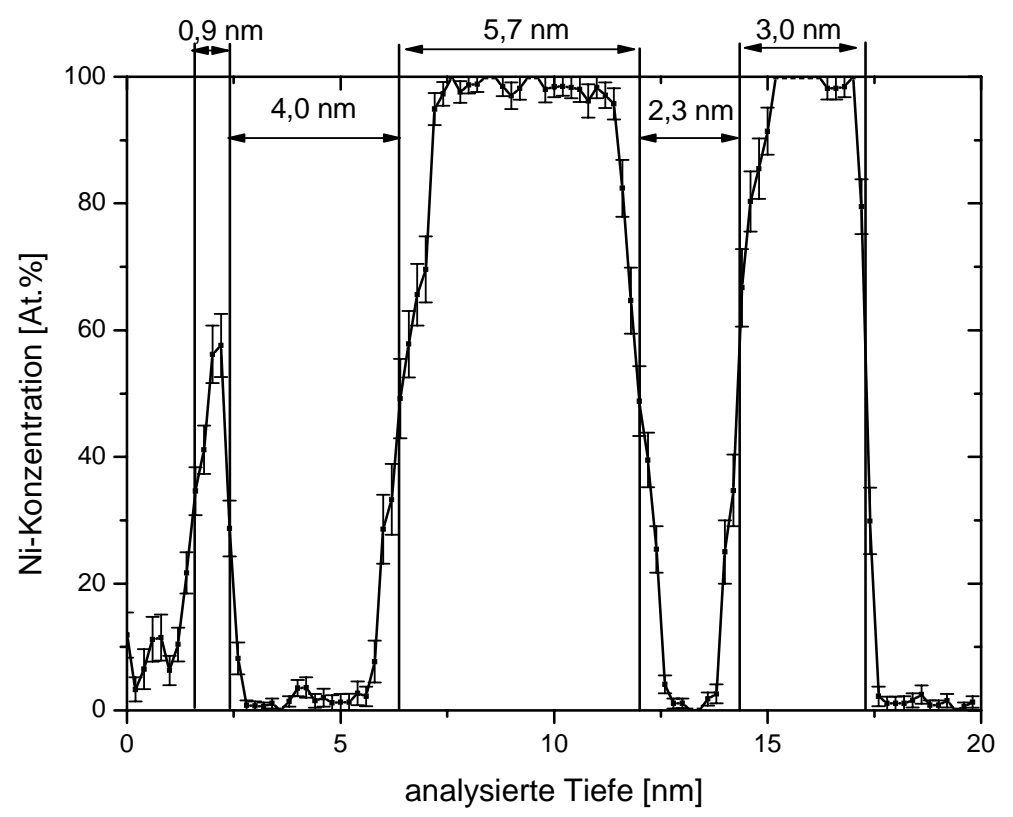

Abb. 4.14: Konzentrationsverlauf durch den in Abb. 4.13 eingezeichneten Zylinder mit einem Radius von $3 \mathrm{~nm}$ und einer Länge von $20 \mathrm{~nm}$.

Der Konzentrationsverlauf bestätigt die Anwesenheit reiner Ni-Körner mit Ausdehnungen von 0,9-5,9 $\mathrm{nm}$. Durch deren plattenförmige Anordnung können bei 
der Konzentrationsbestimmung Artefakte durch den „local magnification effect“ ausgeschlossen werden. Weite Teile der Proben weisen keine Ni-Anreicherungen mehr auf. Stattdessen wird eine homogen verteilte Restlöslichkeit von 0,5 At. \% Ni detektiert. Um die lateralen Ausdehnungen plattenförmiger Ni-Körner ebenfalls erfassen zu können wurden FIM-Untersuchungen durchgeführt. Abbildung 4.15 zeigt zwei FIMBilder einer Ni-Plattenformation, die unmittelbar nacheinander aufgenommen wurden. Das linke Bild zeigt über den Apex der Spitze ausgedehnte, kristalline Ni-Bereiche. Diese Struktur ändert sich bei weiterem Feldverdampfen der Oberflächenatome (rechtes Bild) durch eine Erhöhung der angelegten Spannung schnell, was mit den in TAPAnalysen beobachteten kleinen Abständen der Platten untereinander gut übereinstimmt.
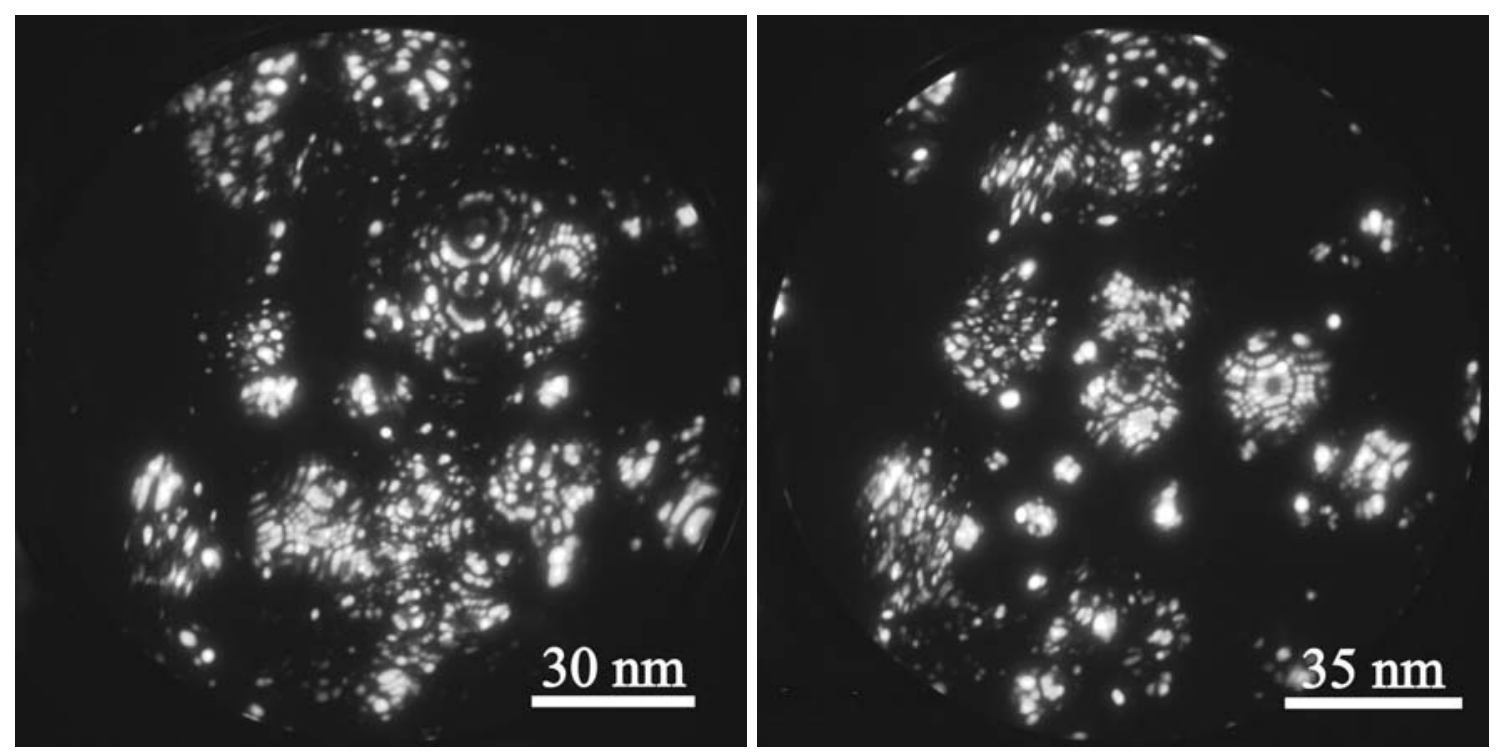

Abb. 4.15: FIM-Bilder einer Probe mit hohem Ni-Gehalt nach einer Auslagerung von $30 \mathrm{~min}$ bei $400{ }^{\circ} \mathrm{C}$; links: weit ausgedehnte Ni-Platten sind vorhanden; rechts: nach Feldverdampfung einiger Atomlagen

Eine Übersicht über die durchgeführten Auslagerungen und beobachteten NiKorngrößen ist in Tabelle 4.1 für die Proben mit hohem Ni-Gehalt zusammengefasst. Es ist festzustellen, dass die Häufigkeit von Mikroabrissen bei den Spitzen im ausgelagerten Zustand deutlich geringer ist. Der geschätzte mittlere Abstand zwischen zwei Poren ist mit $140 \mathrm{~nm}$ etwa doppelt so hoch wie im unbehandelten Zustand. Eine 
Versprödung des Materials durch die durchgeführten Temperaturbehandlungen wurde nicht beobachtet.

\begin{tabular}{|c|c|c|}
\hline Auslagerungszustand & Analysmethode & Ni-Korngröße \\
\hline unbehandelt & FIM / TAP & $3 \pm 2 \mathrm{~nm}$ \\
\hline $10 \mathrm{~min} / 200^{\circ} \mathrm{C}$ & TAP & $7 \pm 3 \mathrm{~nm}$ \\
\hline $30 \mathrm{~min} / 400^{\circ} \mathrm{C}$ & FIM / TAP & $\begin{array}{c}\text { „plattenförmig“ } \\
4 \pm 2 \mathrm{~nm} / 37 \pm 6 \mathrm{~nm}\end{array}$ \\
\hline
\end{tabular}

Tab. 4.1: Übersicht über die untersuchten Zustände der Proben mit hohem Ni-Gehalt

Im Fall der Ag-Ni Proben mit niedrigem Ni-Gehalt wurden, aufgrund des definierteren Ausgangszustandes, kleinere Auslagerungszeiten bei geringeren Temperaturen gewählt. Bereits nach $5 \mathrm{~min}$ bei $200{ }^{\circ} \mathrm{C}$ bilden sich nanometergroße Ni-Körner in der Ag-Matrix (Abb. 4.16).

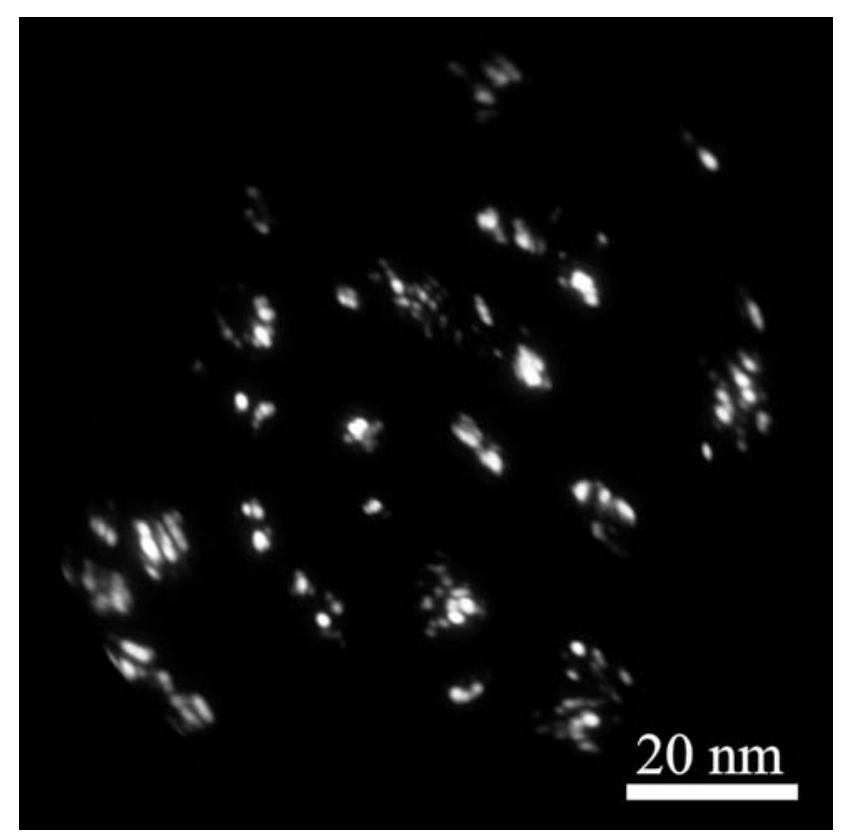

Abb. 4.16: FIM-Bild einer Probe mit niedrigem Ni-Gehalt nach einer Auslagerung von 5 min bei $200{ }^{\circ} \mathrm{C}$; viele kristalline Ni-Körner sind zu erkennen. 
Auslagerungen für $30 \mathrm{~min}$ bei $300^{\circ} \mathrm{C}$ führen $\mathrm{zu}$ einer drastischen Abnahme der Anzahldichte der Ni-Körner, so dass diese mit den eingesetzten Methoden nicht mehr erfasst werden können. Erstaunlicherweise finden sich Agglomerationen von NiAtomen, die zu Ni-Konzentrationsfluktuationen bis $8 \mathrm{At}$. \% führen. Es wird darüber hinaus eine Restlöslichkeit von Ni in Ag von 0,5 $\pm 0,2$ At. \% beobachtet.
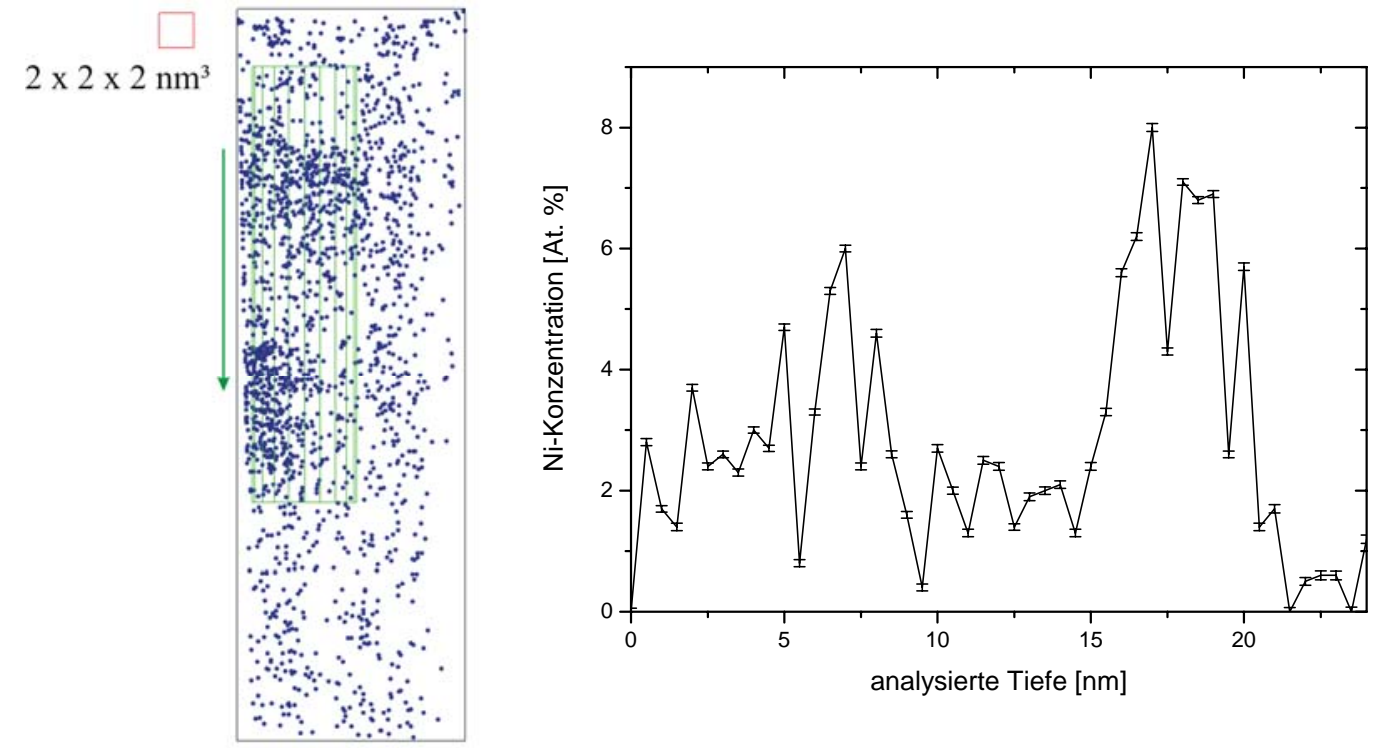

Abb. 4.17: TAP-Analyse einer Probe mit niedrigem Ni-Gehalt nach einer 30 min bei $300^{\circ} \mathrm{C}$ und Analysezylinder Radius $3 \mathrm{~nm}$, Länge $25 \mathrm{~nm}$ (links); Agglomerationen von Ni-Atomen bis 8 At. \% (rechts)

\subsubsection{Ag-Korngrößenentwicklung}

Die Ag-Körner sind aufgrund des starken Phasenkontrastes nur durch TAP-Analysen auflösbar. Es fällt zunächst im direkten Vergleich der unbehandelten Zustände beider untersuchten Ag-Ni-Probenserien auf, dass die Ag-Körner ein lamellares Gefüge mit unterschiedlichen Ausdehnungen bilden. Während die lateralen Abmessungen nahezu identisch sind, werden in den Proben mit niedrigem Ni-Gehalt deutlich längere und etwas schlankere Ag-Körner beobachtet (siehe Tab. 4.2).

Nach einer Wärmebehandlung von $10 \mathrm{~min}$ bei $200^{\circ} \mathrm{C}$ wölben sich die Ag-Korngrenzen in den Proben mit hohem Ni-Gehalt bereits deutlich, so dass die lamellare Struktur des 
Ausgangszustandes bereits weitestgehend zerstört ist. Die nadelförmigen Proben verbiegen sich dabei makroskopisch parallel zur Pressrichtung.

\begin{tabular}{|c|c|}
\hline Probentyp & Ag-Korngröße \\
\hline Ag- $14,3 \mathrm{At} . \% \mathrm{Ni}$ & $2-7 \mathrm{~nm} />30 \mathrm{~nm}$ \\
\hline $\mathrm{Ag}-3,1 \mathrm{At.} \% \mathrm{Ni}$ & $3-5 \mathrm{~nm} />70 \mathrm{~nm}$ \\
\hline
\end{tabular}

Tab. 4.2: Vergleich der Ag-Korngrößen der unbehandelten Ag-Ni-Proben mit hohem und niedrigem $\mathrm{Ni}$-Gehalt

Abbildung 4.18 zeigt ein solches Gefüge, in dem sich eine Ag-Korngrenze mit lokalen Krümmungsradien von etwa $10 \mathrm{~nm}$ um mehrere Ni-Körner wölbt. Nach 30 Minuten bei $400{ }^{\circ} \mathrm{C}$ glätten sich die Ag-Korngrenzen wieder, es sind nur noch leichte Wölbungen mit Krümmungsradien von $25-40 \mathrm{~nm}$ zu beobachten (Abb. 4.19 links). Es sind keine Korrelationen zwischen Ni-Körnern und Korngrenzwölbungen mehr zu erkennen, wie der Vergleich mit der Ni-Verteilung im analysierten Volumen zeigt (Abb. 4.19 rechts).

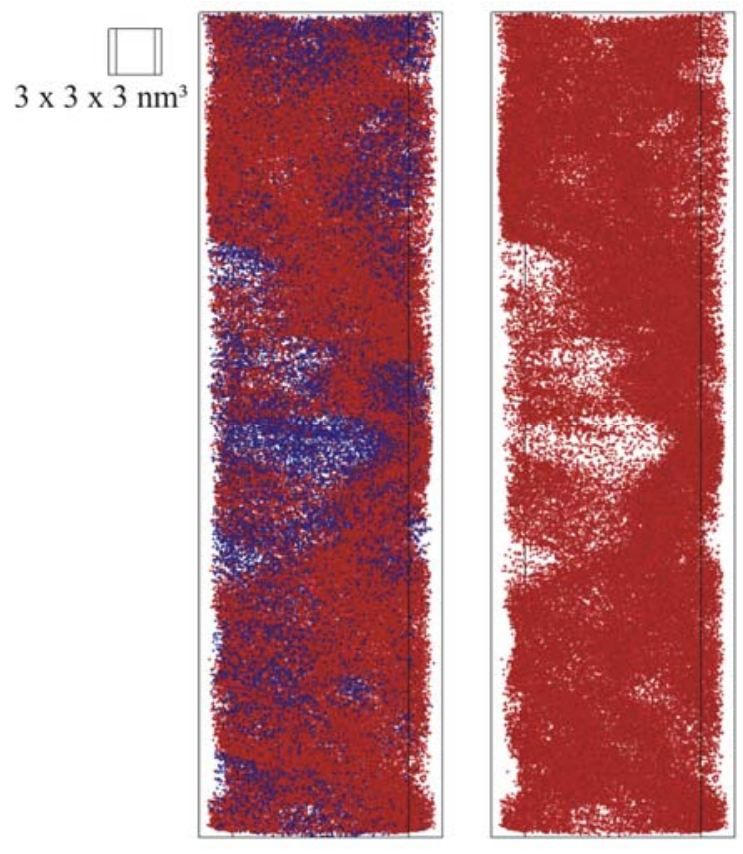

Abb. 4.18: TAP-Analyse einer Probe mit hohem Ni-Gehalt nach $10 \mathrm{~min}$ bei $200{ }^{\circ} \mathrm{C}$; links: nur alle Atome sind dargestellt, eine Korngrenze wölbt sich stark um mehrere NiKörner; rechts: nur Ag-Atome sind dargestellt. 


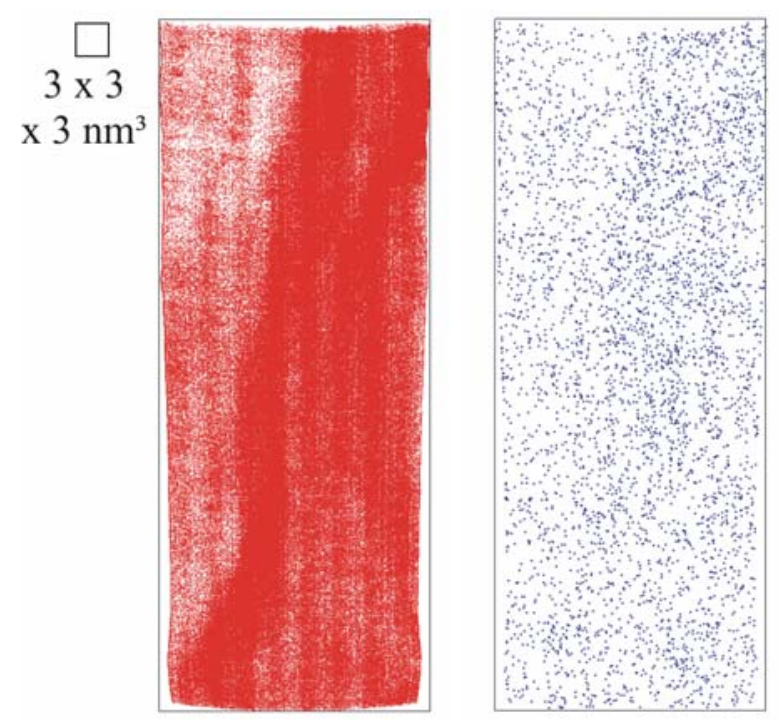

Abb. 4.19: TAP-Analyse einer Probe mit hohem Ni-Gehalt nach 30 min bei $400{ }^{\circ} \mathrm{C}$; links: nur Ag-Atome sind dargestellt, eine leicht gewölbte Korngrenzen ist zu erkennen; rechts: nur Ni-Atome sind dargestellt.

In Proben mit niedrigem Ni-Gehalt wurden keine gewölbten Ag-Korngrenzen beobachtet. Die Ag-Körner waren in allen untersuchten Zuständen lateral bereits so stark gewachsen, dass keine Bestimmung der Korngröße mehr möglich war.

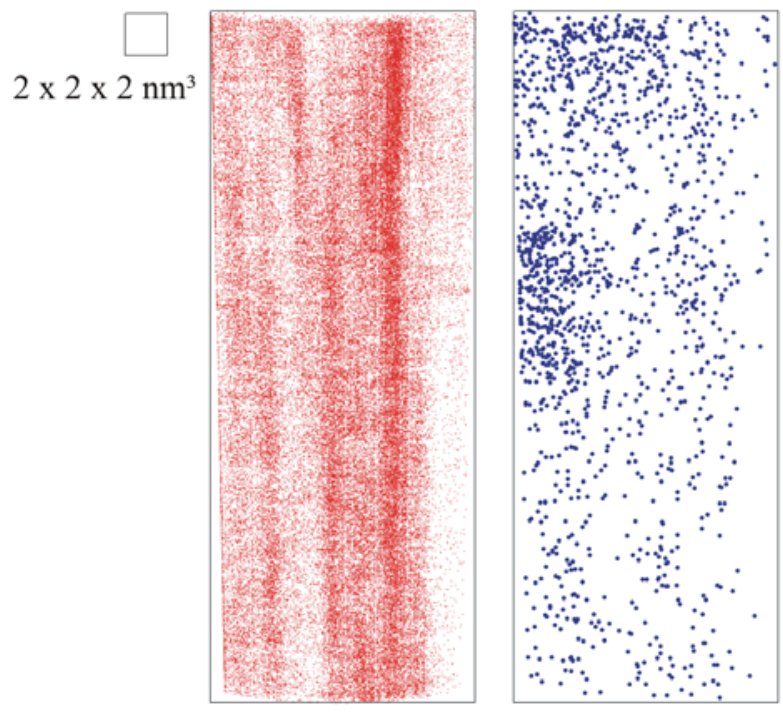

Abb. 4.20: TAP-Analyse einer Probe mit niedrigem Ni-Gehalt nach 30 min bei $300{ }^{\circ} \mathrm{C}$; links: nur Ag-Atome sind dargestellt, die Ag-Korngrenzen weisen keine Wölbung auf; rechts: nur Ni-Atome sind dargestellt. 


\subsection{Untersuchung der Ag-Ni Multilagen}

Es wurden Experimente mit nanokristallinen Ag-Ni Multilagen durchgeführt, um ein abgeschlossenes Bild der Korngrenzsegregation in Ag-Ni Legierungssystem zu erhalten. Diese wurden durch Sputterdeposition direkt auf vorpräparierte W-Substrate aufgebracht (siehe Abschnitt 3.1). Es wurden jeweils Probenchargen mit zwei bis drei Ag-Ni Doppelschichten hergestellt, wobei die Ni-Schichtdicke stets kleiner als fünf [111]-Monolagen gehalten wurde (Abb. 4.21). Im Folgenden werden die Ergebnisse der Auslagerungen bei verschiedenen Temperaturen und Zeiten vorgestellt.

\subsubsection{As-sputtered Zustand}

Die Ag-Ni-Multilagen weisen im Feld-Ionen-Mikroskop das gleiche Kontrastverhalten auf wie die co-evaporierten Proben. Die Ag-Schichten werden durch hellen NiSchichten überstrahlt und erscheinen beim Durchtritt durch die Schichten als schwarze Ringe. Eine Sequenz von FIM-Bildern in Abbildung 4.22 zeigt die Oberfläche einer Probe mit drei Ag/Ni Doppelschichten bei stetiger Erhöhung der angelegten Spannung.

\begin{tabular}{|l|l|c|c|}
\hline \multicolumn{1}{|c|}{ Modus } & Zeit & Beam voltage [V] & Beam current $\left[\mathbf{m A} / \mathbf{c m}^{2}\right]$ \\
\hline Targetreinigung & $3 \mathrm{~min}$ & $600 \mathrm{~V}$ & $2,8 \mathrm{~mA} / \mathrm{cm}^{2}$ \\
\hline Probenreinigung & $45 \mathrm{~s}$ & $500 \mathrm{~V}$ & $1,4 \mathrm{~mA} / \mathrm{cm}^{2}$ \\
\hline Beschichtung & $\begin{array}{l}\mathrm{Ag} 40 \mathrm{~s} \\
\mathrm{Ni} 20 \mathrm{~s}\end{array}$ & $550 \mathrm{~V}$ & $1,7 \mathrm{~mA} / \mathrm{cm}^{2}$ \\
\hline
\end{tabular}

Tab. 4.3: Parameter für die sputterdeponierten Ag-Ni Schichten 


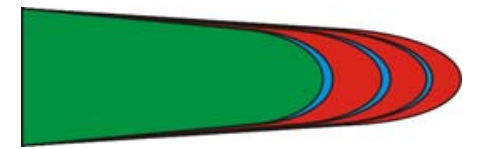

Abb. 4.21: Schematische Darstellung des hergestellten Multilayersystems; Farbschlüssel: $\mathrm{Ag} \mathrm{Ni} W$

Die erste Ag-Schicht ist aufgrund ihres dunklen Kontrastes nicht sichtbar. Die Sequenz beginnt daher mit der ersten Ni-Schicht (a), die von einem schnellen Durchbruch der zweiten Ag-Schicht (b) gefolgt wird. Nach weiterer Feldverdampfung von Ag wird die zweite Ni-Schicht sichtbar (c) und breitet sich über den Apex der Spitze aus (d-e). Bedingt durch die wesentlich kleinere Feldverdampfungsfeldstärke von Ag treten durch $\mathrm{zu}$ träge Spannungsanpassung bei manueller Spannungsregulierung oft unkontrollierte Durchbrüche durch eine Ag-Schicht auf. Dies ist im Fall der dritten Ag-Schicht (f) geschehen. Deutlich erkennt man die schalenartige Struktur von hellen Ni- und dunklen Ag-Bereichen. Die weitere Bildsequenz (g-i) zeigt den Übergang auf das $<110>$-texturierte W-Substrat. Das epitaktische Aufwachsen der (111)-Atomlagen des $\mathrm{Ni}$ ist an den Ringstrukturen deutlich zu erkennen. Alle Ni-Schichten weisen eine Ringstruktur auf, die der $<111>$-Textur entspricht. Diese bleibt über die Ag-Schichten hinweg erhalten, woraus auf eine starke Epitaxie geschlossen werden kann.

Um mehr Informationen über die Korngrenzen und Grenzflächen zu erhalten, wurden TAP-Analysen durchgeführt. Abbildung 4.23 zeigt eine solche Analyse des gleichen Schichtpaket-Typs. 

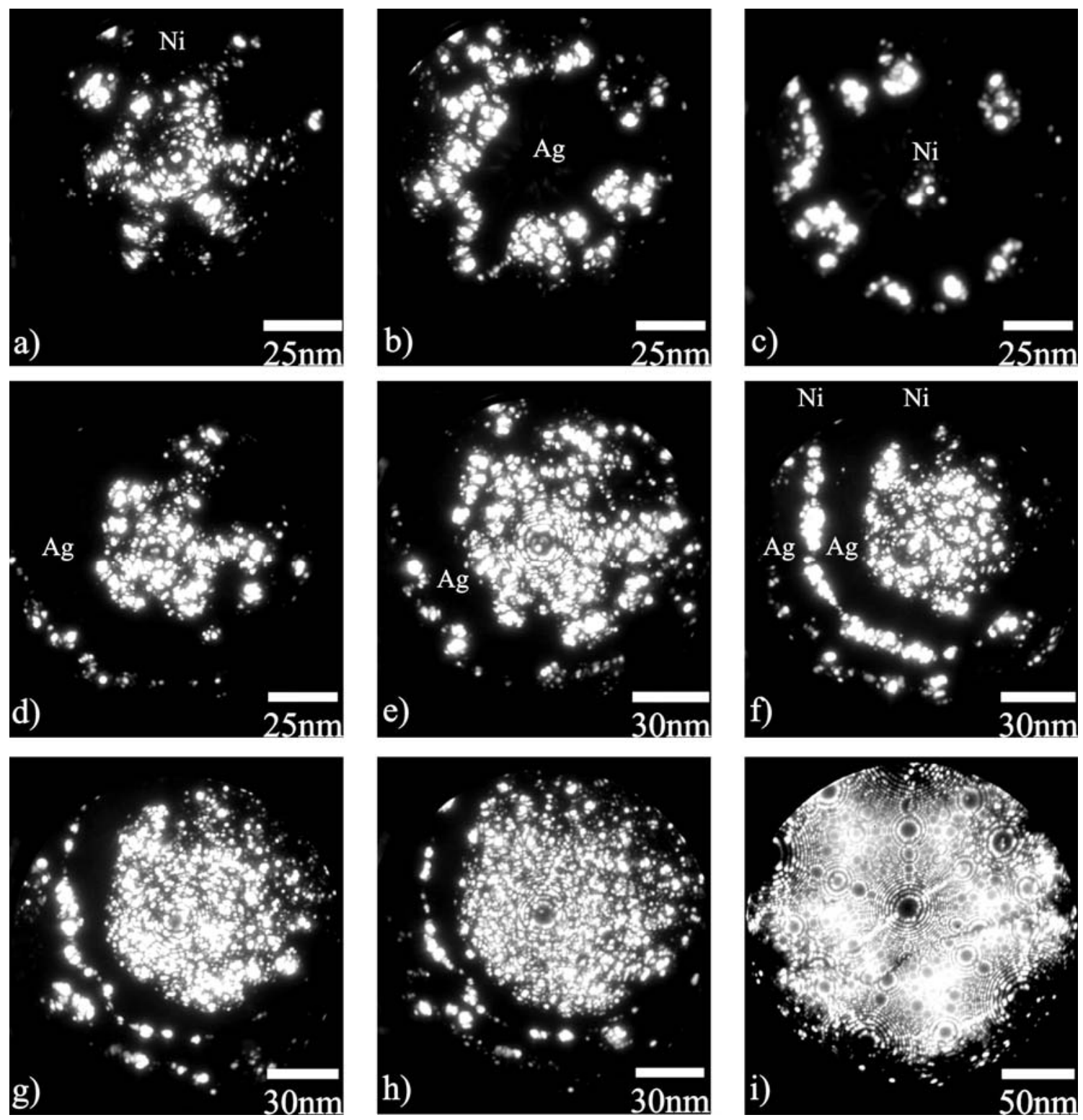

Abb. 4.22: FIM-Bildersequenz bei stetiger Erhöhung der Spannung; die Ag-Schichten (dunkel) und Ni-Schichten (hell) werden von a) bis i) kontinuierlich abgetragen; $T=30 \mathrm{~K}$, Bildgas Ne $3 \cdot 10^{-5}$ mbar

Ein Analysezylinder wurde im rekonstruierten Volumen so positioniert, dass alle Schichten senkrecht durchstoßen wurden. Die Konzentrationsverläufe beider gesputterter Elemente Ag und Ni, sowie W des Substrats sind in Abbildung 4.24 entlang des Zylinders (in Abb. 4.23 von oben nach unten) mit den jeweiligen Schichtbreiten aufgetragen. Diese variieren bei gleichen Sputterparametern offensichtlich mit dem während der Deposition abnehmenden Krümmungsradius der Substratspitze. Die Dicke der durchmischten Zone der Multilagen kann ebenfalls aus den Konzentrationsverläufen 
ermittelt werden. Die Breite der Ag/Ni Grenzfläche kann im Mittel mit $\sim 0,5 \mathrm{~nm}$ angegeben werden und ist daher als nicht durchmischt anzusehen.
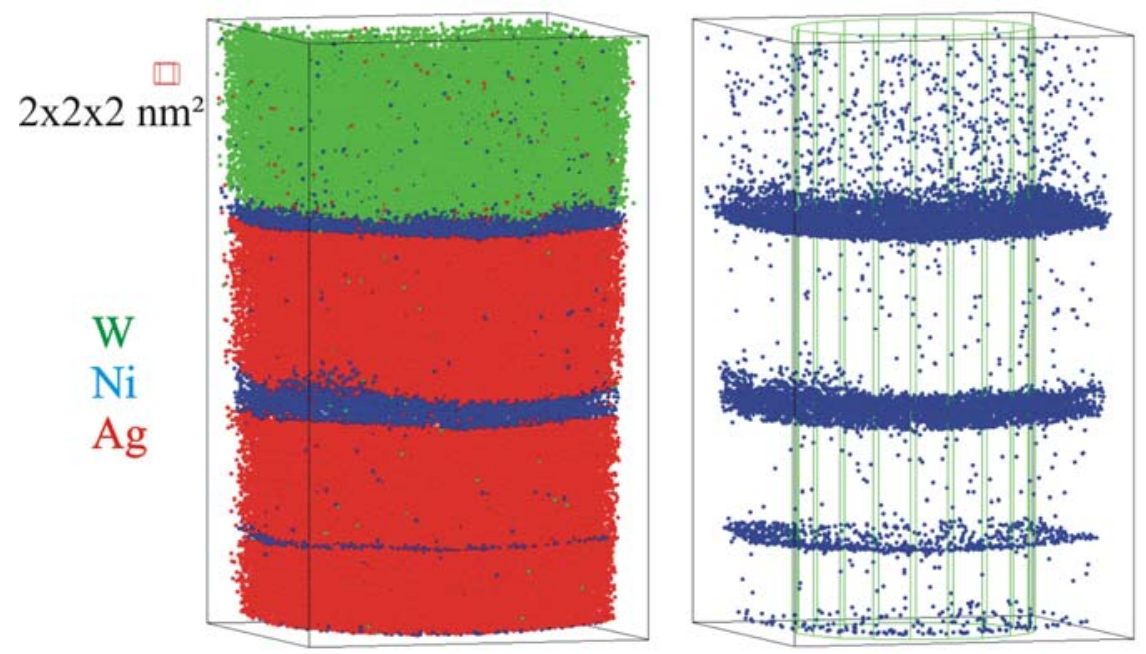

Abb. 4.23: Wie hergestelltes, unbehandeltes Schichtpaket; links: alle Atome dargestellt; rechts: nur Ni-Atome mit Analysezylinder(Radius $6 \mathrm{~nm}$ )

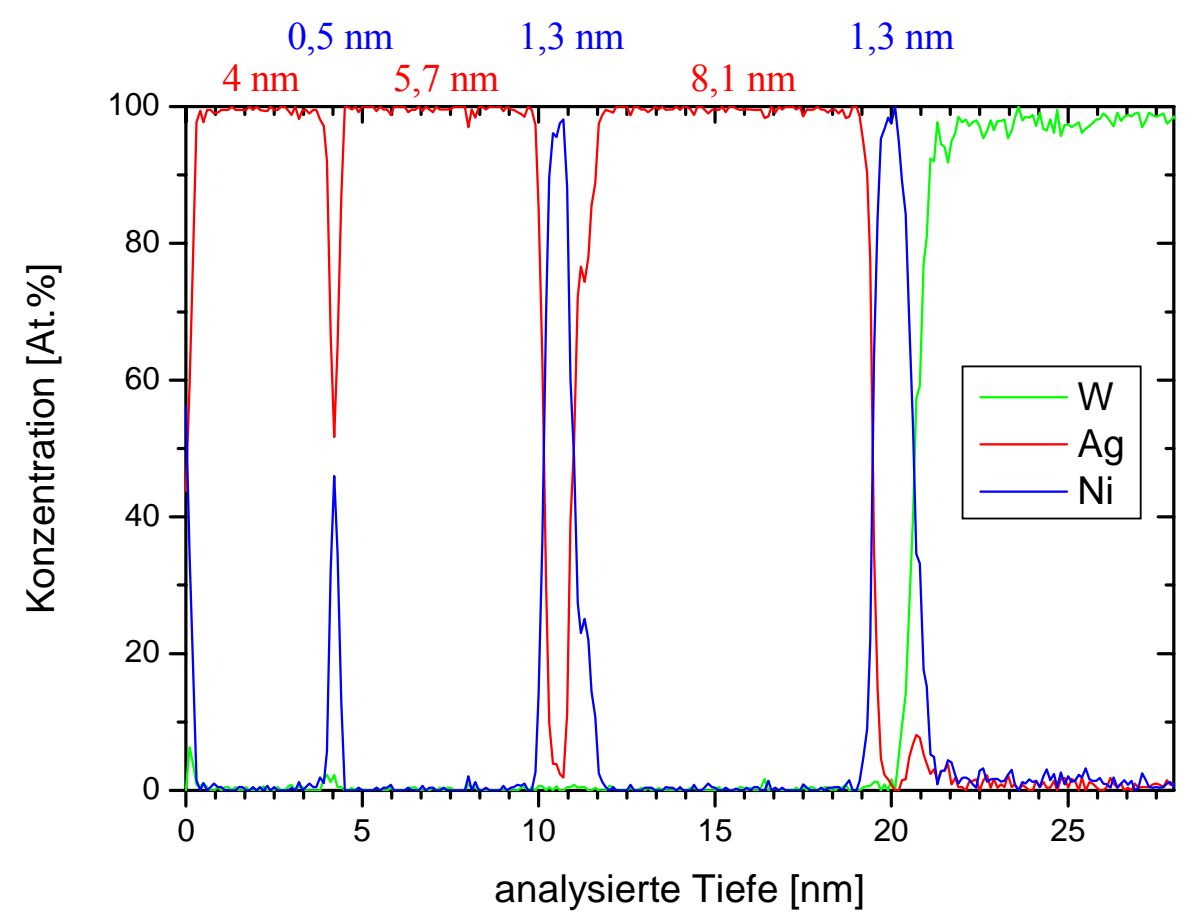

Abb. 4.24: Konzentrationsverlauf durch drei Ag-Ni Doppelschichten mit den jeweiligen Schichtdicken 


\subsubsection{Zerfall der Multilagen}

Zunächst wurden die Temperaturbehandlungen mit den gleichen Parametern wie bei den co-evaporierten Ag-Ni-Proben durchgeführt, um einen direkten Vergleich der Ergebnisse zu ermöglichen. Da die Schichtpakete, im Gegensatz zu den co-evaporierten Proben, bei Temperaturen um $200^{\circ} \mathrm{C}$ keinerlei Reaktionen zeigten, wurden für die weiteren Untersuchungen Auslagerungen für verschiedene Zeiten bei $300{ }^{\circ} \mathrm{C}$ und $400{ }^{\circ} \mathrm{C}$ gewählt. Es erwies sich aufgrund der mit der Sputterdauer abnehmenden Einzelschichtdicke als sinnvoll, diese Experimente mit nur zwei Ag/Ni-Doppelschichten durchzuführen. Abbildung 4.25 zeigt ein für $30 \mathrm{~min}$ bei $300^{\circ} \mathrm{C}$ ausgelagertes Schichtpaket. Eine Korngrenze verläuft senkrecht durch eine Ag-Schicht und verbindet beide Ni-Schichten. Dies bestätigt das erwartete, kolumnare Wachstum von Schichten auf dem gekrümmten W-Substrat.

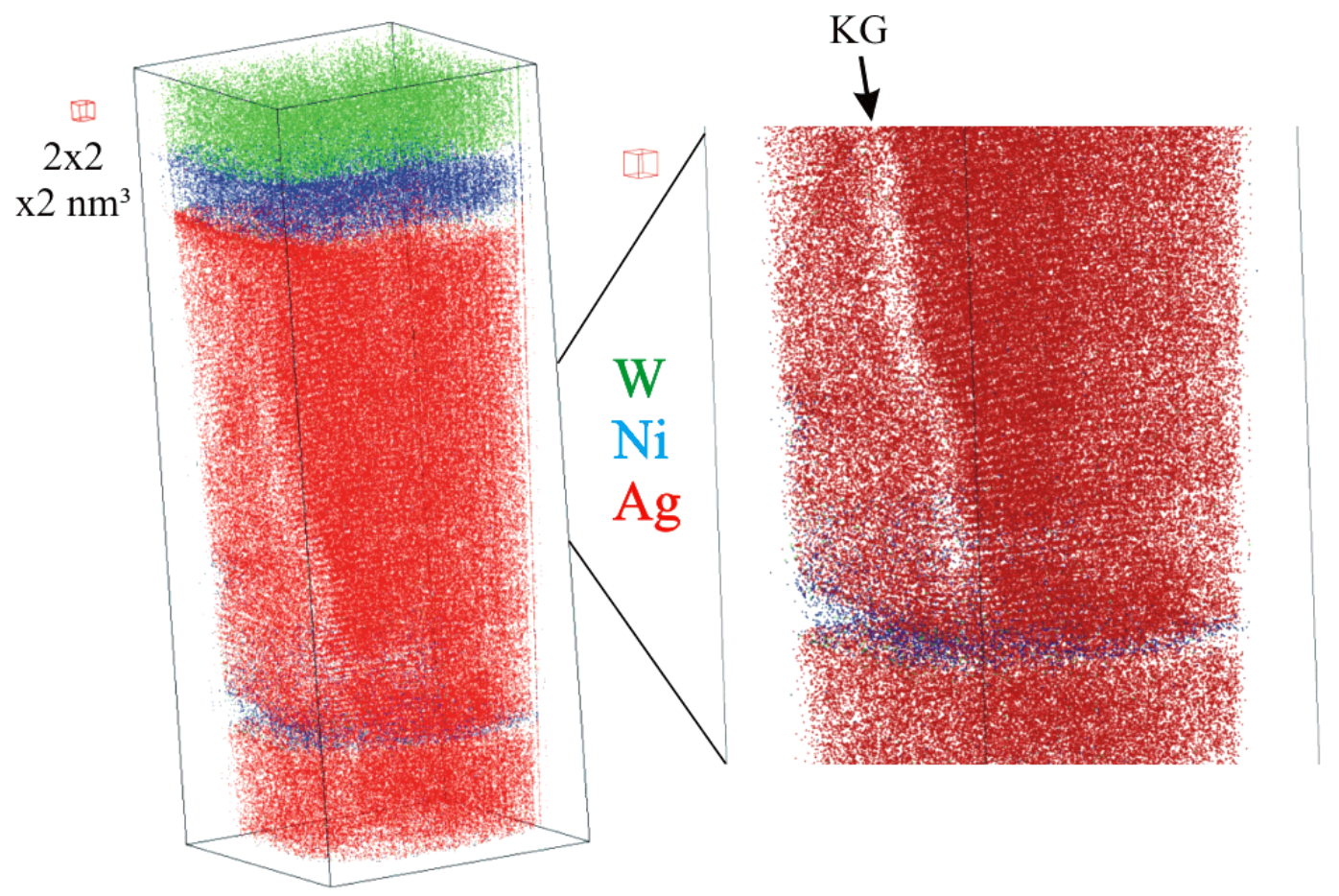

Abb. 4.25: TAP-Analyse eines Schichtpaketes nach einer Temperaturbehandlung von 30 min bei $300{ }^{\circ} \mathrm{C}$; links: gesamte Messung; rechts: Ausschnittsvergrößerung einer AgKorngrenze mit Atomlagen 
Die Breite der Ag/Ni-Grenzfläche ist im Vergleich zum unbehandelten Zustand unverändert. Entlang der Ag-Korngrenze hat keine Eindiffusion oder Segregation stattgefunden, obwohl die Korngrenze an beiden Enden von Ni begrenzt wurde.
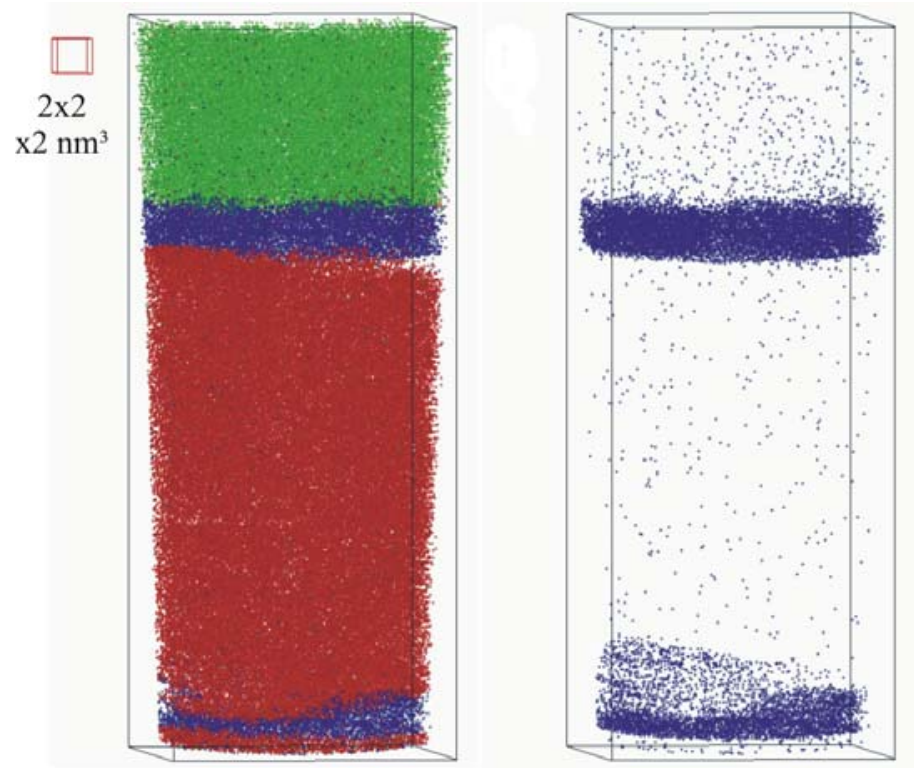

Abb. 4.26: TAP-Analyse eines Schichtpaketes nach einer Temperaturbehandlung von $1 \mathrm{~h}$ bei $300^{\circ} \mathrm{C}$; links: alle Atome; rechts: nur Ni-Atome

Nach $1 \mathrm{~h}$ bei $300^{\circ} \mathrm{C}$ ist an den Proben festzustellen, dass die Dicke der ersten AgSchicht auf etwa ein Drittel reduziert ist (Abb. 4.26 links). Oft wird beobachtet, dass die erste Ni-Schicht leichte Wölbungen hat, wobei aber die Grenzflächenschärfe unverändert bleibt. Nach $30 \min$ bei $400^{\circ} \mathrm{C}$ ist eine Messung der Schichten nur noch in Einzelfällen möglich. Die ersten beiden Schichten fehlten, auch ein Messen bis in das W-Substrat hinein war nicht mehr möglich (Abb. 4.27). Scheinbar geht die für eine TAP-Messung benötigte Spitzengeometrie verloren. Dies wird durch Untersuchungen der nicht messbaren Spitzen belegt (Abb. 4.28). Das SEM-Bild zeigt eine vollständig zerstörte Spitze, wie sie für alle Auslagerungszeiten $>30 \mathrm{~min}$ bei $400{ }^{\circ} \mathrm{C}$ beobachtet wurde. EDX-Messungen bestätigen, dass sich die Multilagen vom Apex der W-Spitze zu den Schaftbereichen zurückgezogen haben. 


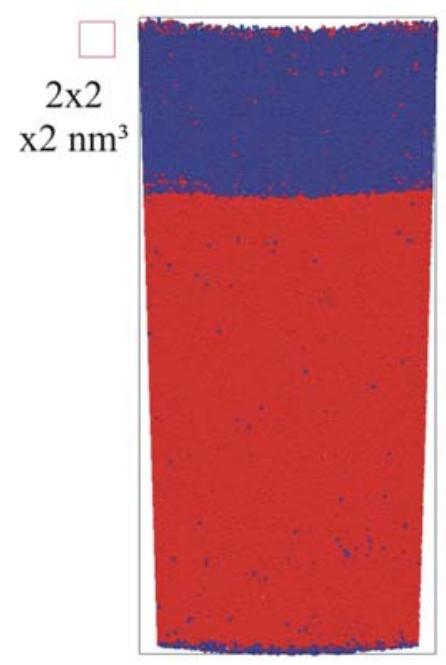

Abb. 4.27: TAP-Analyse eines Schichtpaketes nach einer Temperaturbehandlung von 30 min bei $400{ }^{\circ} \mathrm{C}$
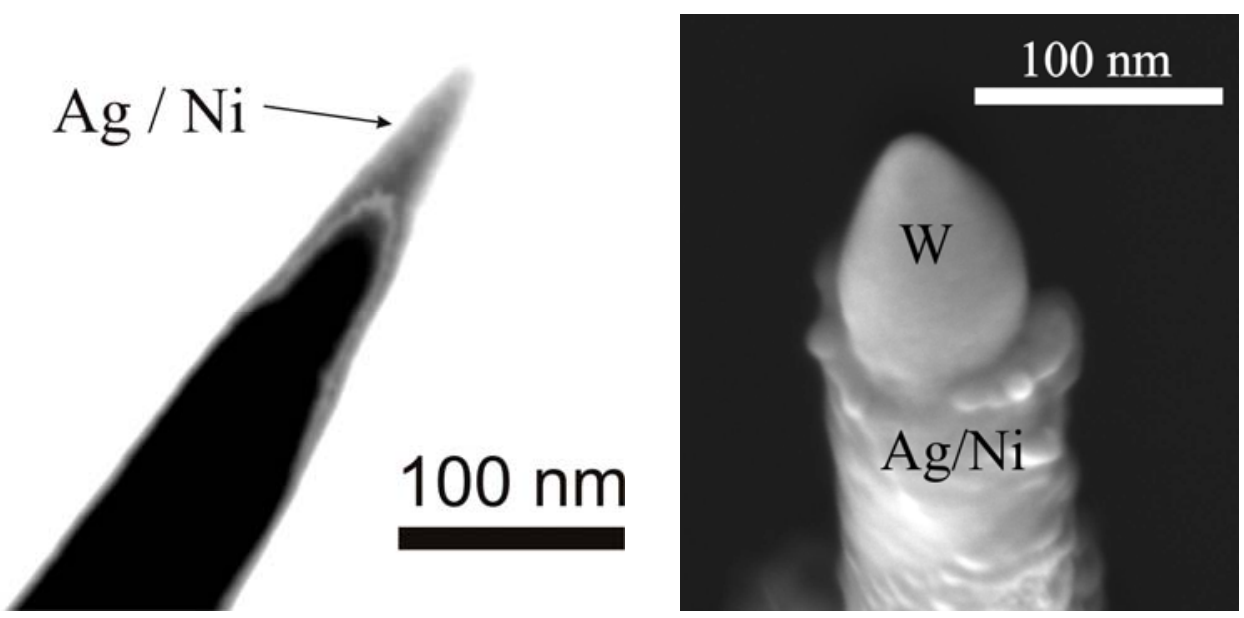

Abb. 4.28: Gesputterte Ag-Ni-Probe; links: TEM-Bild des „, as-prepared“-Zustandes; rechts: SEM-Bild einer nach einer Temperaturbehandlung von $1 \mathrm{~h}$ bei $400{ }^{\circ} \mathrm{C}$ 


\subsection{Untersuchung der sputter-deponierten Cu-Bi-Legierung}

Zur Untersuchung des Cu-Bi-Legierungssystems wurden ebenfalls nanokristalline Schichten erzeugt. Es kamen zunächst mehrere Targettypen zum Einsatz, von denen letztlich die von einem Komposittarget hergestellten Proben die besten Ergebnisse erzielten. Diese werden im Folgenden präsentiert.

\subsubsection{As-sputtered Zustand}

Es zeigte sich bei der Herstellung vieler Proben, dass die im Target angebotene Legierungszusammensetzung, bzw. die angebotenen Flächen von $\mathrm{Cu}$ und $\mathrm{Bi}$, nicht im 1:1 Übertrag auf die deponierte Schicht übergehen. Tabelle 4.4 gibt eine Übersicht über die Bi-Überträge auf das Substrat ${ }^{12}$. Der Bi-Übetrag ist um ein bis zwei Größenordnungen kleiner als es bei der Targetzusammensetzung erwartet wird. Dies wird auf Resputtervorgänge während des Depositionsprozess zurückgeführt (siehe Anhang 7.1).

\begin{tabular}{|l|c|c|}
\hline Targettyp & nominelle Konz. & Bi-Übertrag auf Substrat \\
\hline I Legierung & $\mathrm{Cu}-1 \mathrm{At} . \% \mathrm{Bi}$ & $<$ Detektionsschwelle (TAP) \\
\hline II Komposit Winkel & $\mathrm{Cu}-5 \mathrm{At} . \% \mathrm{Bi}$ & $<$ Detektionsschwelle (TAP) \\
\hline III Komposit Gitter & $\mathrm{Cu}-15 \mathrm{At} . \% \mathrm{Bi}$ & $0-1,5 \mathrm{At} . \%$ \\
\hline IV Komposit Gitter & $\mathrm{Cu}-50 \mathrm{At.} \% \mathrm{Bi}$ & $2-5 \mathrm{At} . \%$ \\
\hline
\end{tabular}

Tab. 4.4: Übersicht über die Bi-Überträge auf das Substrat

Es gelang auch in mehreren Durchläufen und Proben derselben Charge kaum, eine einheitliche Bi-Konzentration in der Bi-Schicht zu erhalten. Die Schichten des Targets III wiesen die geringsten Konzentrationsunterschiede von 0 bis $1,5 \mathrm{At}$ \% auf und

\footnotetext{
${ }^{12}$ Die Targettypen sind ausführlich in Abschnitt 3.1 vorgestellt.
} 
wurden daher als Grundlage für die weiteren Untersuchungen verwendet. Aufgrund einer mangelnden Stabilität der $\mathrm{Cu}(\mathrm{Bi})$-Schicht auf dem W-Substrat wurde eine 2 - $3 \mathrm{~nm}$ dicke Co-Schicht als Haftvermittler verwendet. Die Abbildung 4.29 zeigt die Anordnung der präparierten Multilagen.

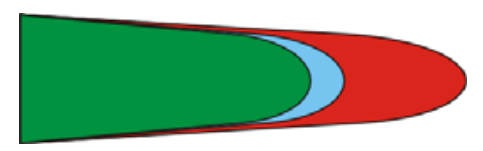

Abb. 4.29: Schematische Darstellung des hergestellten Multilayersystems; Farbschlüssel: $\mathrm{Cu}(\mathrm{Bi}) \mathrm{Co} W$

Eine Sequenz von FIM-Bildern (Abb. 4.30) zeigt die Nanostruktur der Cu(Bi)-Schicht. Die Dichte der Großwinkel-Korngrenzen nimmt in die Tiefe der Schicht kontinuierlich zu. Dies kann auf ein kolumnares Wachstum der Körner beim Depositionsprozess auf die Spitze zurückgeführt werden. Einige Körner überwachsen dann mit zunehmender Schichtdicke benachbarte Körner, wobei an der durch FIM beobachteten Oberfläche bei größerer Schichtdicke weniger Großwinkel-Korngrenzen hervortreten. Auch Kleinwinkel-Korngrenzen werden beobachtet (Abb. 4.30 blaue Pfeile). Die Herstellung eines nanokristallinen $\mathrm{Cu}(\mathrm{Bi})$-Gefüges durch die Sputter-Deposition war somit erfolgreich. Aufgrund der niedrigeren Feldverdampfungsfeldstärke des $\mathrm{Bi}$ von $18 \mathrm{~V} / \mathrm{nm}$, gegenüber $\mathrm{Cu}$ mit $30 \mathrm{~V} / \mathrm{nm}$, werden Bi-Anreicherungen mit einem dunklen Kontrast abgebildet. Dies macht eine qualitative Aussage über die Bi-Segregation an Korngrenzen, die bereits in reinem $\mathrm{Cu}$ dunkel abbilden, durch FIM-Analysen allerdings unmöglich. 

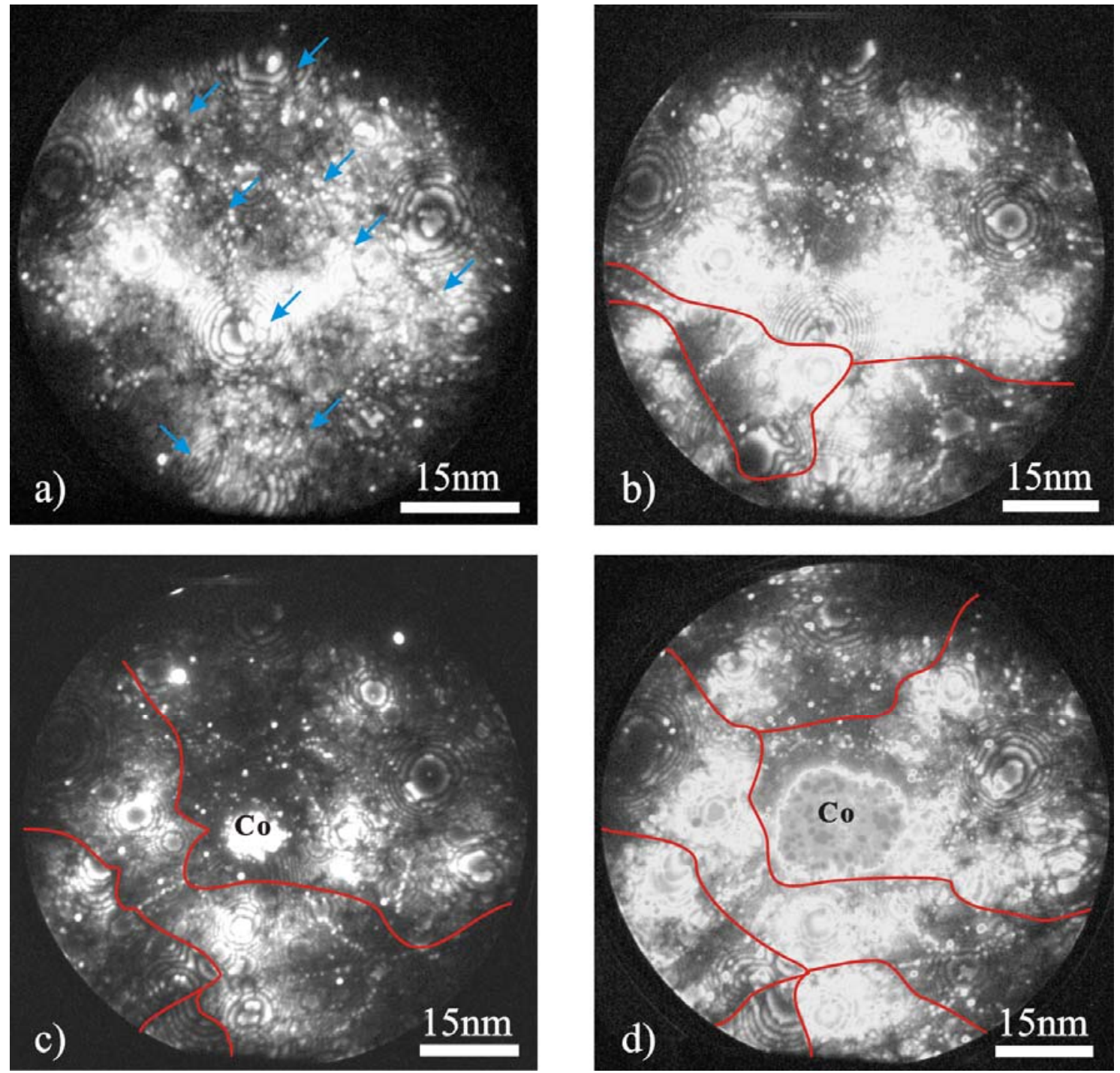

Abb. 4.30: FIM-Bildersequenz bei stetiger Erhöhung der Spannung; die Cu(Bi)-Schicht wird bis in die Co-Schichten von a) bis d) kontinuierlich abgetragen; Pfeile:Kleinwinkel-Korngrenzen; rote Markierungen: Großwinkel-Korngrenzen; $T=30 \mathrm{~K}$, Bildgas Ne $3 \cdot 10^{-5} \mathrm{mbar}$ 


\subsubsection{Exzess an Kleinwinkelkorngrenzen}

TAP-Analysen wurden durchgeführt, um eine quantitative Aussage über die BiSegregation an den Cu-Korngrenzen machen zu können. Die Abbildung 4.31 (Mitte) zeigt eine Messung des unbehandelten ,as-sputtered“-Zustandes.

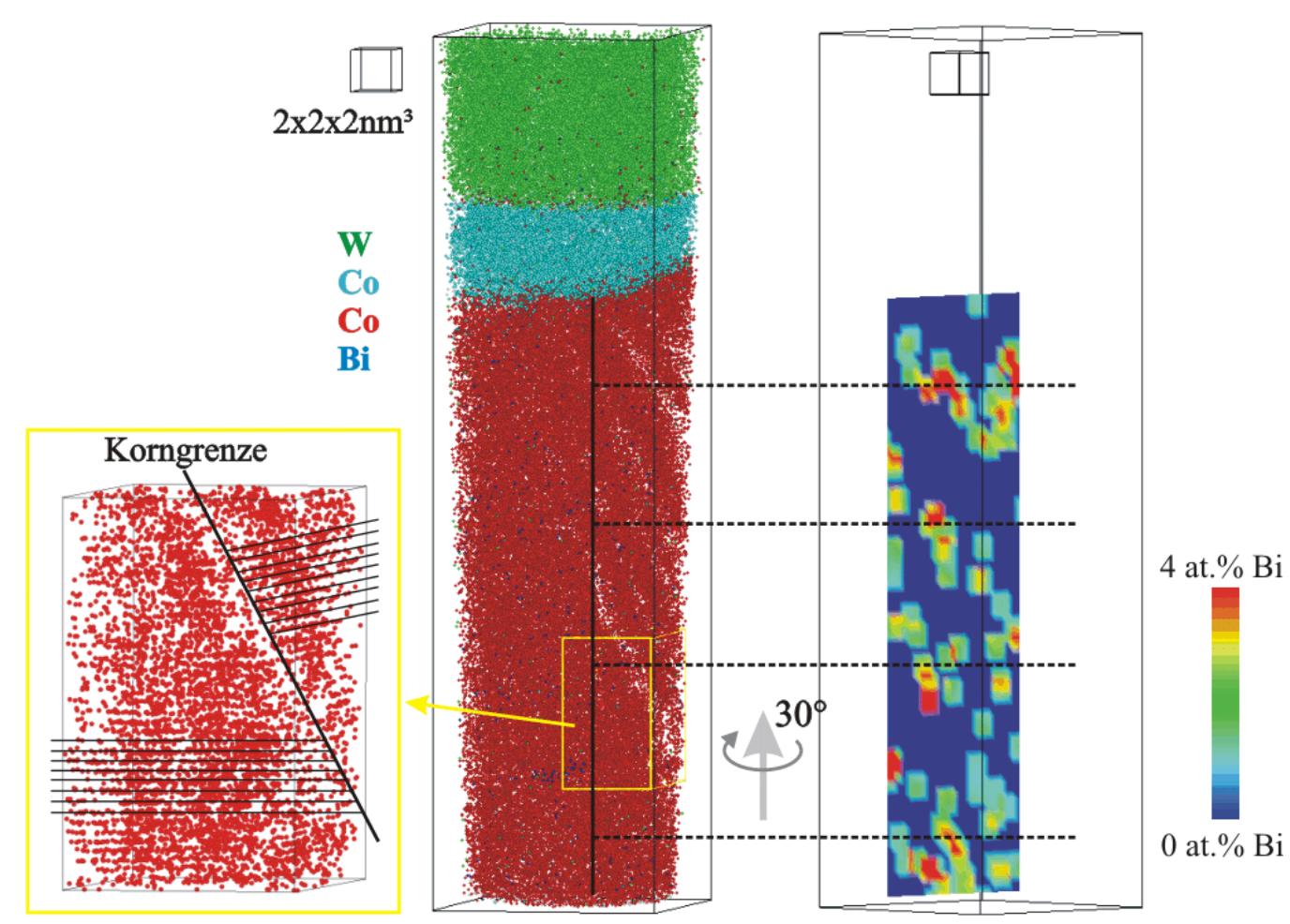

Abb. 4.31: Links: Ausschnittsvergrößerung; Mitte: Rekonstruiertes TAP-Volumen; rechts: TAP-Volumen um $30^{\circ}$ im Uhrzeigersinn gedreht mit Konzentrationsdarstellung; Die zugehörige Schnittebene der Konzentrationsverteilung und gestrichelte Linien zur Orientierung sind verzeichnet.

Die Zusammensetzung der gesamten $\mathrm{Cu}(\mathrm{Bi})-\mathrm{Schicht}$ beträgt 1,2 (0,2) At. \% Bi. Eine lamellenartige Struktur mit linienförmigen Bereichen geringerer Dichte ist zu erkennen. Ein diese Struktur enthaltender Quader wurde ausgeschnitten und vergrößert (Abb. 4.31 links). Es werden beidseitig der linienhaften Zone reduzierter Dichte Atomlagen mit einem Kippwinkel von $\sim 12^{\circ}$ aufgelöst. Die Zonen reduzierter Dichte sind somit als 
Kleinwinkel-Kippkorngrenzen identifiziert. Bereits im „as-sputtered“ Zustand werden durch ein Konzentrationsverteilungs-Bild Bi-Anreicherungen mit Konzentrationen über $4 \mathrm{At} . \% \mathrm{Bi}$ an diesen Korngrenzen gefunden (Abb. 4.31 rechts). Es besteht dabei eine gute Übereinstimmung zwischen den schräg im Analysevolumen liegenden Korngrenzen und dem durch die Ebene angeschnittenen Exzess an der Korngrenze. Über einen Analysezylinder (Abb. 4.32) mit einem Durchmesser von 1,5 $\mathrm{nm}$ und einer Länge von $10 \mathrm{~nm}$ wurde ein Leiterdiagramm durch ein $\mathrm{Cu}-$ Korn und zwei Korngrenzen aufgetragen (Abb. 4.33).

$2 \times 2 \times 2 \mathrm{~nm}^{3}$

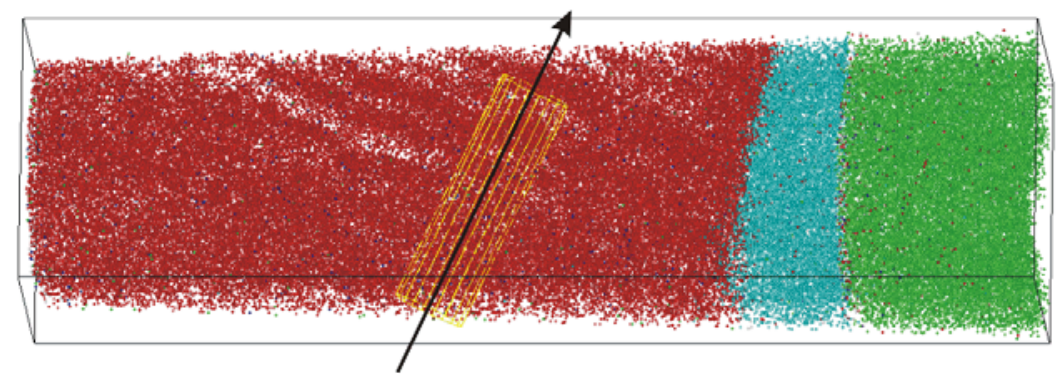

Abb. 4.32: TAP-Volumen; Analysezylinder (gelb) mit einem Radius von 1,5 $\mathrm{nm}$ und einer Länge von $10 \mathrm{~nm}$

Für die thermodynamische Beschreibung der Segregation ist der Bi-Exzess, also die Überschussanzahl der Bi-Atome gegenüber der Restlöslichkeit der Körner, von großem Interesse. Der Exzess ist von der Korngrenzbreite unabhängig definiert als (vgl. Kap. $1.1 .2)$

$$
\Gamma_{B i}=\frac{N_{B i}}{A}
$$

Mit Hilfe der angelegten Tangenten lassen sich die überschüssigen Bi-Atome in der Korngrenze abzählen. Bezogen auf die Grundfläche $A=\pi r^{2}$ des Analysezylinders bestimmt sich dann der Korngrenzenexzess aus $\Gamma_{B i}=N_{B i} /\left(\pi r^{2}\right)$, wobei beim Wert von $N_{B i}$ die Detektionseffizienz von $S_{D} \sim 0,5$ der tomographischen Atomsonde zu berücksichtigen ist. 


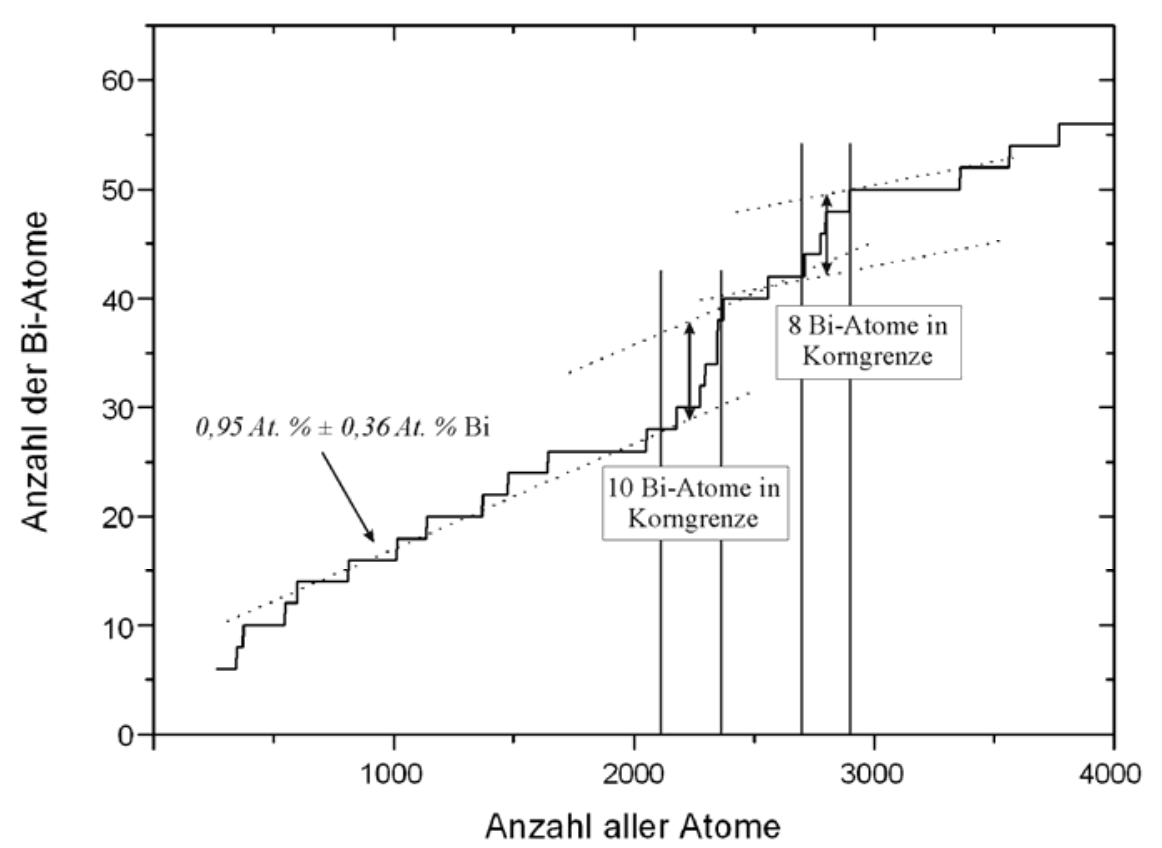

Abb. 4.33: Leiterdiagramm durch ein Cu-Korn und zwei Korngrenzen (die Detektionseffizienz wurde bereits berücksichtigt); Zustand „, as-sputtered“

Aus fünf gemessenen Maximalwerten (Tab. 4.5) von $\Gamma_{B i}$ ergibt sich mit $r=1,5 \mathrm{~nm}$ ein mittlerer Korngrenzenexzess von $\Gamma_{B i}=1,3$ Atome $/ \mathrm{nm}^{2}$.

\begin{tabular}{|l|c|c|c|c|c|c|}
\hline$N_{B i}$ & 10 & 8 & 6 & 12 & 9 & Mittelwert \\
\hline$\Gamma_{B i}\left[\right.$ Atome $\left./ \mathrm{nm}^{2}\right]$ & 1,4 & 1,1 & 0,8 & 1,7 & 1,3 & 1,3 \\
\hline
\end{tabular}

Tab. 4.5: Korngrenzen-Exzess-Werte unbehandelter Proben

Die Steigung entspricht im Leiterdiagramm der Konzentration im Molenbruch. Im ersten Teil des Leiterdiagramms wurde diese Konzentration in einem Cu-Korn bestimmt. Mit einer Konzentration von $c_{B i, K o r n}=0,95$ At. \% Bi ist die $\mathrm{Cu}-\mathrm{Schicht}$ nach der Deposition hoch übersättigt (siehe Phasendiagramm Abb. 1.4). 
Die Gesamtkonzentration setzt sich aus Beiträgen der Körner und der Korngrenzen zusammen. Es gilt:

$$
c_{B i}=\frac{N_{B i}}{N_{C u}+N_{B i}}=\frac{A \cdot \Gamma_{B i}+N_{B i, \text { Korn }}}{N_{C u}+N_{B i}}=\frac{A \cdot \Gamma_{B i} \cdot V_{M}}{V}+c_{B i, K o r n}
$$

wobei $V_{M}=7,11 \cdot 10^{-6} \mathrm{~m}^{3}$ das Molvolumen von $\mathrm{Cu}$ und $A$ die gesamte Korngrenzfläche im Probenvolumen $V$ sind. Unter der Annahme sphärischer oder kubischer $\mathrm{Cu}-\mathrm{Körner}$ kann ihr Oberflächen-zu-Volumenverhältnis mit $A / V=3 / g$ beschrieben werden ${ }^{13}$. Somit kann der mittlere Korndurchmesser g berechnet werden:

$$
g=\frac{3 \cdot \Gamma_{B i} \cdot V_{M}}{c_{B i}-c_{B i, \text { Korn }}}
$$

Mit den angegebenen Werten für $c_{B i}, c_{B i \text {,Korn }}$ und $\Gamma_{B i}$ errechnet sich so ein Wert von $g=18 \mathrm{~nm}$, der gut mit den in Abbildung 4.30 dargestellten Körnern übereinstimmt. Abweichungen von der sphärischen bzw. kubischen Form sowie statistische Schwankungen der eingesetzten Werte bedingen allerdings einen großen Fehler des $g$-Wertes.

Auslagerungszustände dieser Proben konnten aufgrund starker Versprödungserscheinungen nicht erfolgreich analysiert werden. Die Gründe für das Versagen dieser stark an $\mathrm{Bi}$ übersättigten Proben wurden daraufhin anhand der bikristallinen $\mathrm{Cu}-\mathrm{Bi}-\mathrm{Proben}$ untersucht. Die Ergebnisse werden in den folgenden Abschnitten dargestellt.

\footnotetext{
13 Die gemeinsamen Oberflächen benachbarter Körner werden von diesem Modell nur einfach berücksichtigt.
} 


\subsection{Bi dotierte $219 a$ Bikristalle}

Die für diese Arbeit hergestellten Bi-dotierten Bikristalle bieten die Möglichkeit der gezielten Untersuchung eines Korngrenztyps. Neben vielen Vorteilen, die der Einsatz der Atomsondentomographie zur Analyse bietet, ergibt sich als Nachteil eine aufwendige Probenpräparation. Die Anwesenheit der $\Sigma 19 a$ Korngrenze in der präparierten Spitze soll zunächst verifiziert werden. Anschließend werden die an diesen Proben gewonnenen Ergebnisse vorgestellt.

\subsubsection{Verifizierung der $219 a$ Korngrenze}

Im Folgenden soll der Erfolg der angewandten Präparationsmethode demonstriert werden. Zu diesem Zweck wurden die hergestellten Spitzen im TEM untersucht.
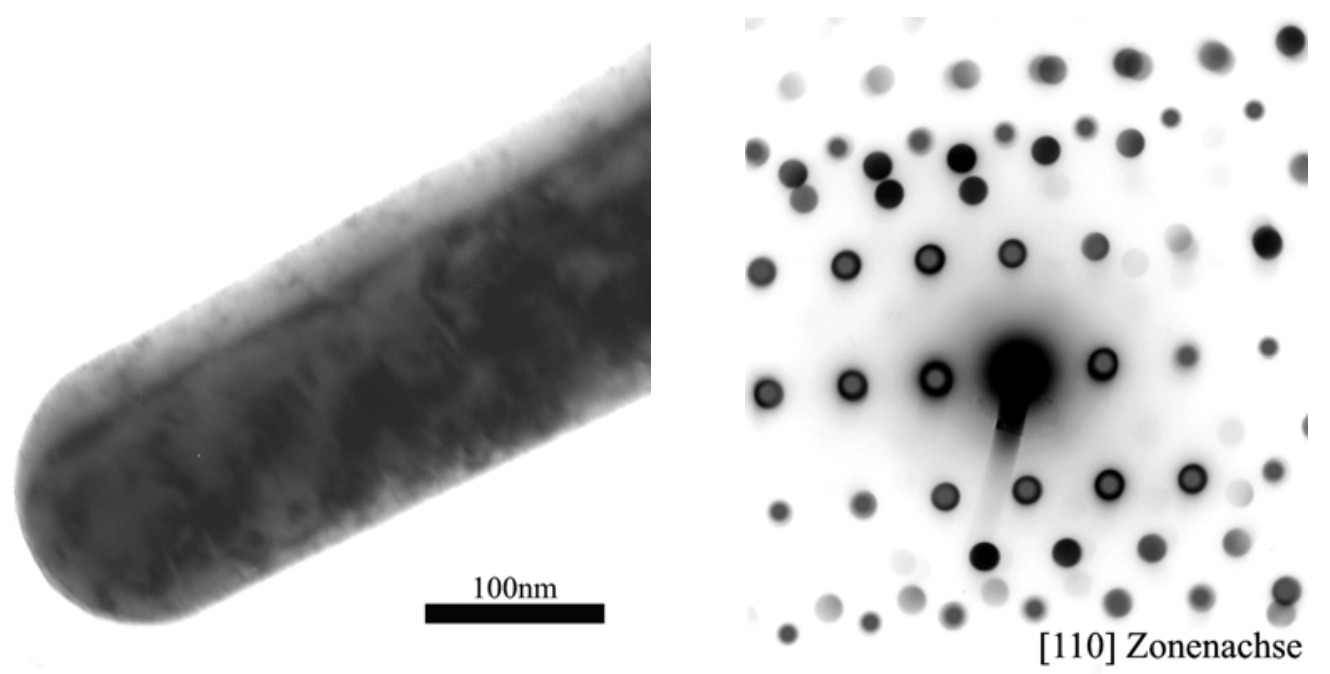

Abb. 4.34: TEM-Bilder einer präparierten bikristallinen Probe; links Realbild; rechts: zugehöriges Beugungsbild

Unter einem geeigneten Winkel erhält man einen Orientierungskontrast und ist somit in der Lage, ein einzelnes Korn des Bikristalls zu durchstrahlen. Die Abbildung 4.34 links zeigt das Realbild des geeignet orientierten Spitzenapex. Der Verlauf der Korngrenze 
entlang der Spitzenachse ist durch den unterschiedlichen Kontrast der beiden Körner deutlich zu erkennen. Das zugehörige Feinbereichs-Beugungsbild (Abb. 4.34 rechts) bestätigt durch zwei übereinander liegende Punktmuster die Anwesenheit der Korngrenze in der präparierten Spitze. Die scheinbare Krümmung der Korngrenze am Apex ist durch ein Artefakt der Abbildung begründet. Blickt man auf eine zur Elektronenstrahlrichtung verkippte Korngrenze, folgt bei Transmission durch nur eine Kristallhälfte ein abknickendes Verhalten des abgebildeten Korngrenzverlaufes am hemisphärischen Spitzenapex. Da dieser Effekt aufgrund geometrischer Überlegungen erwartet wurde, kann dies ebenfalls als Beweis des Vorhandenseins der Korngrenze betrachtet werden.

\subsubsection{Bi-Versprödung der Korngrenze}

Die Bi-Versprödung erwies sich im Fall der 120 ppm Bi-dotierten Bikristalle als ein erhebliches Problem. Diese zerbrachen teilweise beim Abschrecken der Proben nach der Segregationsbehandlung von $12 \mathrm{~h}$ bei $850^{\circ} \mathrm{C}$. Die verbliebenen Bikristalle brachen bei leichtesten mechanischen Beanspruchungen auseinander. Die Bruchfläche war metallisch glänzend und ohne jegliche Stufen, was deutlich auf einen Bruch entlang der aufwändig präparierten Korngrenze hinweist. Leider entziehen sich diese Proben aufgrund des Bruches der weiteren Untersuchung mit FIM/TAP und TEM.

Die 40 ppm Bi-dotierten Bikristalle wiesen auch nach mechanischen Beanspruchungen keinerlei Versprödung auf und konnten daher mit den gewählten Methoden untersucht werden.

\subsubsection{Bestimmung des Korngrenzenexzesses}

Mit einer Einwaage von 40 ppm Bi liegt die Bi-Konzentration der Bikristalle deutlich unter der Nachweisgrenze der tomographischen Atomsonde von 350-200 ppm. Aus diesem Grund beschränkt sich die Analyse ausschließlich auf Bi-Anreicherungen. Eine Bestimmung der Restlöslichkeit von $\mathrm{Bi}$ in $\mathrm{Cu}$ ist nicht möglich. 

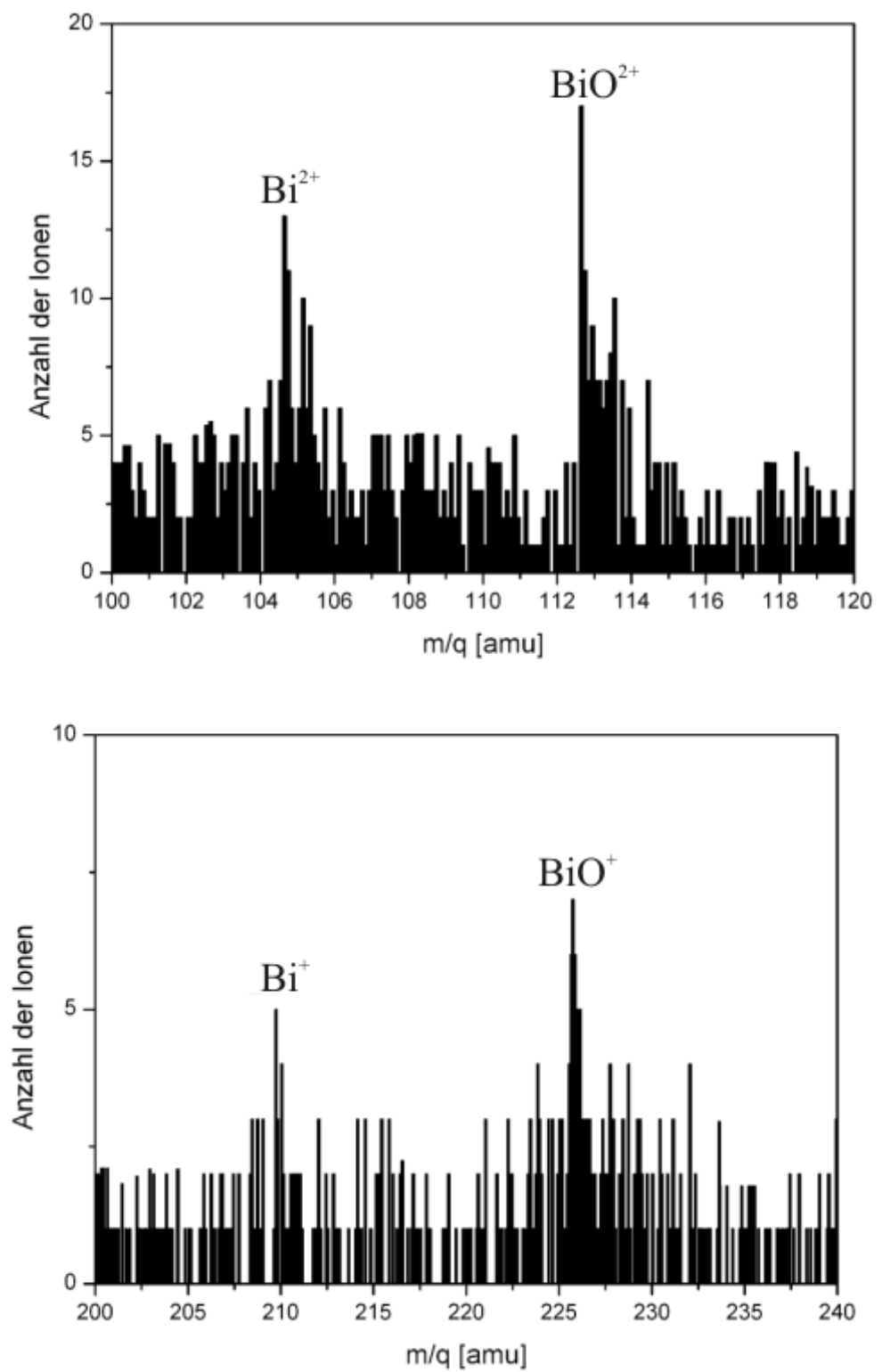

Abb. 4.35: Ausschnitte des TAP-Massenspektrums einer Bikristallinen Cu-40 ppm Bi Probe nach einer Segregationsbehandlung von $12 \mathrm{~h}$ bei $850{ }^{\circ} \mathrm{C}$

Das Massenspektrum einer Bikristallinen $\mathrm{Cu}-40 \mathrm{ppm} \mathrm{Bi}$ Probe zeigt nach der Segregationsbehandlung ein signifikantes Bi-Signal. Abbildung 4.35 zeigt Peaks einfach und zweifach geladener Bi-Ionen und jeweils die zugehörigen Oxid-Peaks. Die entsprechende Rekonstruktion des Volumens zeigt eine flächige Anreicherung von BiAtomen im analysierten TAP-Volumen (Abb. 4.36 links). Eine Ebene wurde parallel zur Analyserichtung in das Volumen eingezeichnet und mit einem farbigen Konzentrationsschlüssel versehen. Es werden Konzentrationen bis $3 \mathrm{At}$. \% beobachtet. 
Die Anreicherung setzt sich in die Tiefe der Messung fort, wie es ausgehend von der präparierten Korngrenzlage parallel zur Spitzenachse zu erwarten ist.
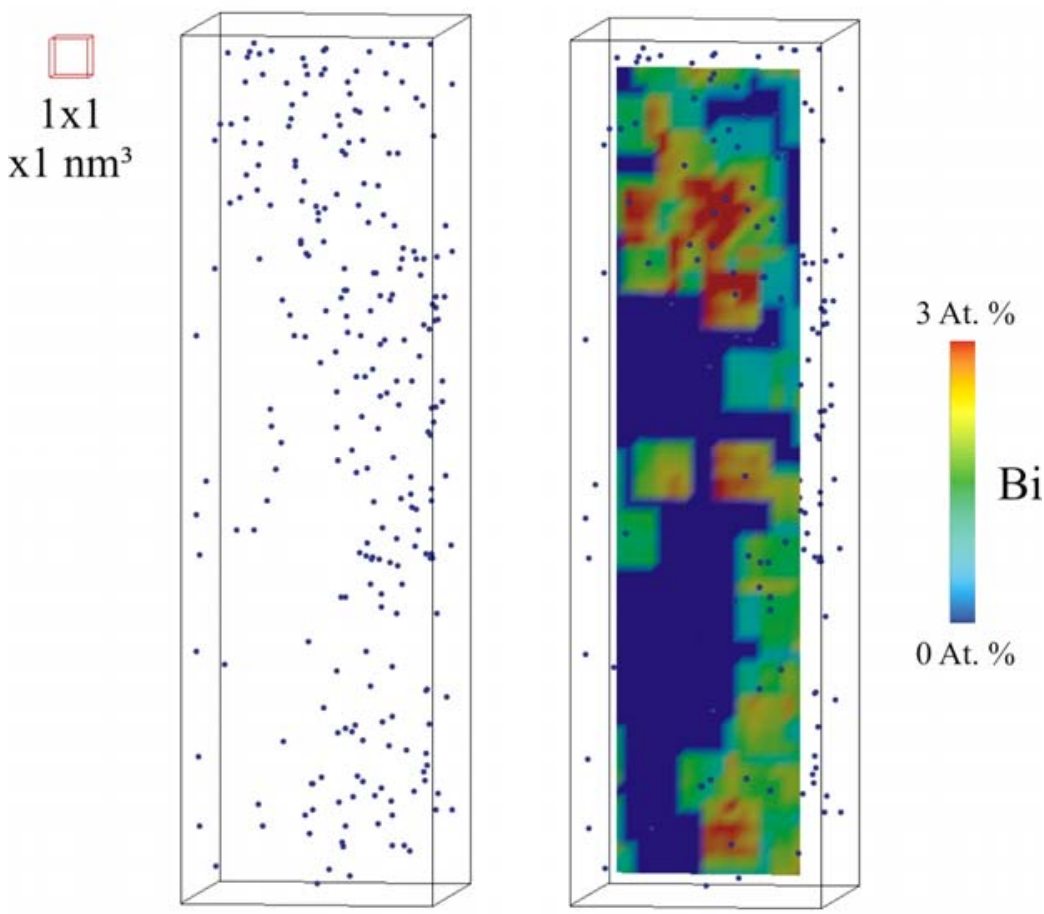

Abb. 4.36: TAP-Analyse des Cu-Bi-Bikristalls nach der Segregationsbehandlung; links: nur Bi-Atome (blau); rechts: Bi-Atome und senkrechte Ebene durch das Volumen mit Konzentrationen; eine flächige Anreicherung von Bi mit bis 3 At. \% ist vorhanden.

Aufgrund der experimentellen Befunde kann somit die Anwesenheit der zuvor durch FIB präparierten $\Sigma 19 a$-Korngrenze auch in TAP-Analysen bestätigt werden. Diese wird durch das Analysevolumen in ihrem Verlauf angeschnitten. Die Breite der Segregationszone beträgt 2-3 $\mathrm{nm}$. Die Fläche des an Bi angereicherten Bereichs beträgt im TAP-Volumen $148 \mathrm{~nm}^{2}$. Diese wird der Korngrenze zugeordnet. Durch Zählen aller detektierten Bi-Atome im Volumen kann unter Berücksichtigung der Detektionseffizienz von $50 \%$ der Bi-Korngrenzexzess $\mathrm{zu} \Gamma_{B i}=3,2 \pm 0,5$ Atome $/ \mathrm{nm}^{2}$ bestimmt werden. Dieser Wert liegt deutlich höher als die gemessenen Exzesswerte an den gesputterten Schichten. 


\subsubsection{Facettierung an der Korngrenze}

Häufig wurde in Vorgängerarbeiten [ALBER99], [SIGLE02] für höher und etwas niedriger Bi-dotierte bikristalline Proben eine Facettierung der Korngrenze nach einer Segregationsbehandlung mit Facettenbreiten von $5 \mathrm{~nm}$ bis $100 \mathrm{~nm}$ festgestellt. Aus den bikristallinen Proben wurden daher mittels FIB auch für diese Arbeit dünne TEMLamellen präpariert, die die $\Sigma 19 a$-Korngrenze enthalten. Eine Facettierung senkrecht zur Durchstrahlrichtung kann jedoch für diesen Zustand ausgeschlossen werden, wie die HR-TEM-Bilder bei verschiedenen Vergrößerungen an unterschiedlichen Probenstellen zeigen (Abb. 4.37). Eine Facettierung, wie sie in Abbildung 4.38 eingezeichnet ist, wäre bei den präparierten Lamellen nicht zu erkennen. In den genannten Vorgängerarbeiten wurde jedoch eine solche Facettierung beobachtet. Somit kann mit diesen neuen Ergebnissen eine Facettierung entlang der $<110>$-Richtung für die vorliegende BiKonzentration von $40 \mathrm{At}$. ppm ausgeschlossen werden.
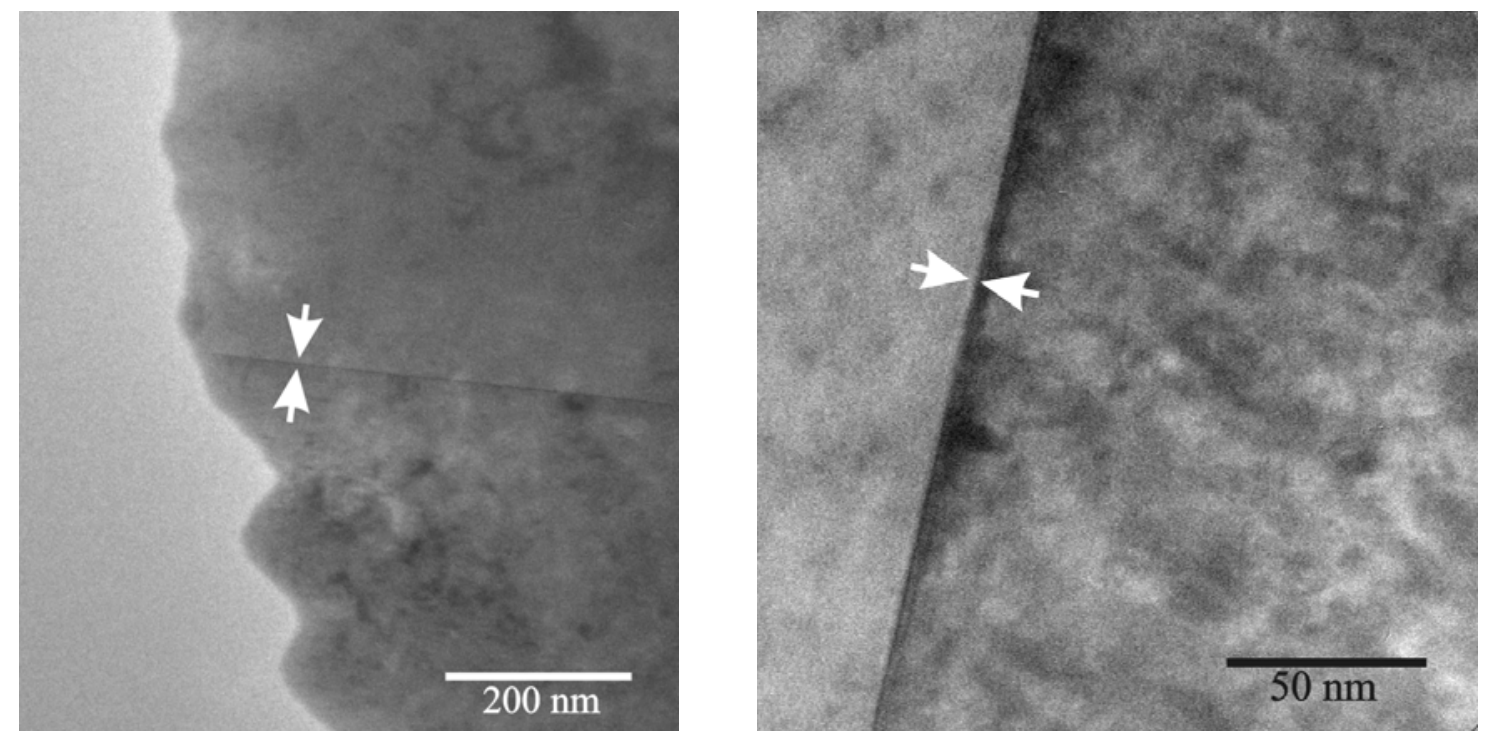

Abb. 4.37: HR-TEM Bilder verschiedener Vergrößerungen der I19a-Korngrenze (Pfeile) nach der Segregationsbehandlung

Es wurde darüber hinaus versucht, die lokale Bi-Konzentration am Ort der Korngrenze mit EDX-Messungen zu bestimmen. Der $200 \mathrm{eV}$ Elektronenstrahl wurde parallel zur 
Korngrenze ellipsoid aufgeweitet um beim Scan mehr Intensität bei geringerer lokaler Erwärmung der Probe zu erzielen. Dieses Vorhaben scheiterte, da auch bei dieser Vorgehensweise eine schnelle Verflüchtigung des Bi eintrat. Bereits nach zwei integralen Scans verschwand das Bi-Signal im Röntgenspektrum. Eine quantitative Aussage war daher nicht möglich. Hier liegt die Vermutung nahe, dass auch die auf EDX-Messungen basierenden Vorgängerarbeiten auf vergleichbare Probleme gestoßen sein müssen. Möglicherweise ist es hilfreich, die Beschleunigungsspannung der Elektronen zu verringern, was jedoch zu einer Verringerung der Punktauflösung des verwendeten Geräts führt.

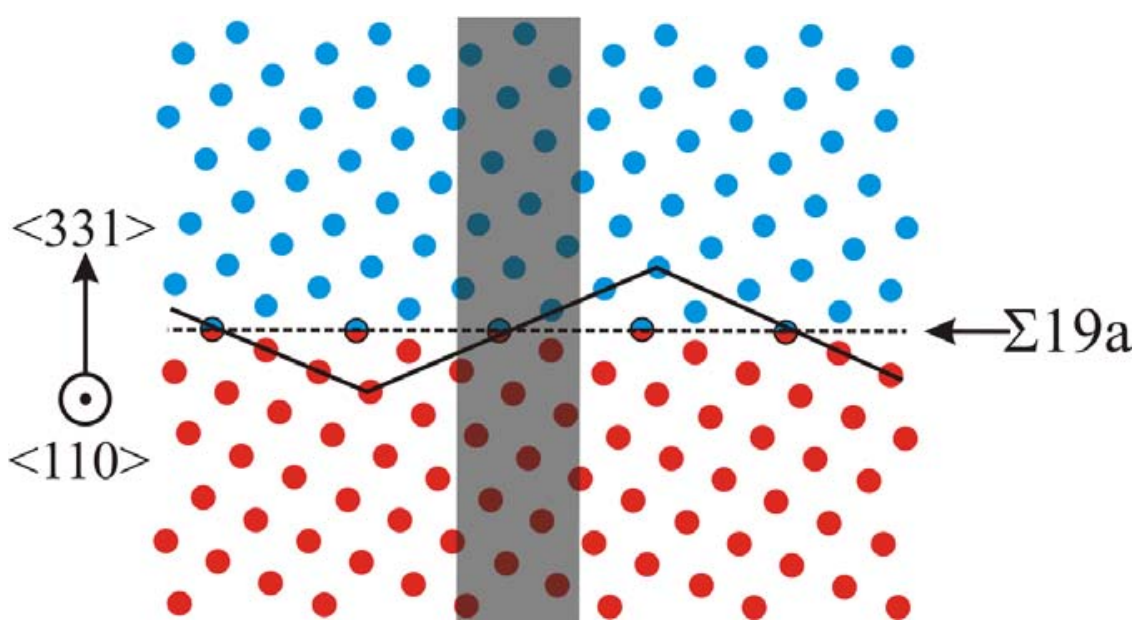

Abb. 4.38: Aufsicht auf die Korngrenze in <110>-Richtung; Entnahmestelle der TEMLamelle (grau); eingezeichnet ist eine unbeobachtbare Facettierung (durchgezogene Linie), wie sie in Vorgängerarbeiten beobachtet wurde.

Häufig waren die bikristallinen Rohlinge bei der Präparation der FIM/TAP-Proben nicht geeignet um zu einer Spitze geschnitten zu werden. Sie enthielten viele Lunker, durch die die Korngrenze verläuft. Eine genauere Betrachtung der Lunker zeigt deutliche Facetten parallel zur Korngrenze und in einem Winkel von $45^{\circ}$ (Abb. 4.39). Die Korngrenze ist leicht zur spitzen Kante des Lunkers gewölbt. Die mittlere Lunkergröße beträgt $320 \pm 90 \mathrm{~nm}$ bei einer mittleren Anzahldichte von 3,3 $10^{9} \pm 0,7 \cdot 10^{9}$ Lunker $/ \mathrm{m}^{2}$ (Abb. 4.40). 

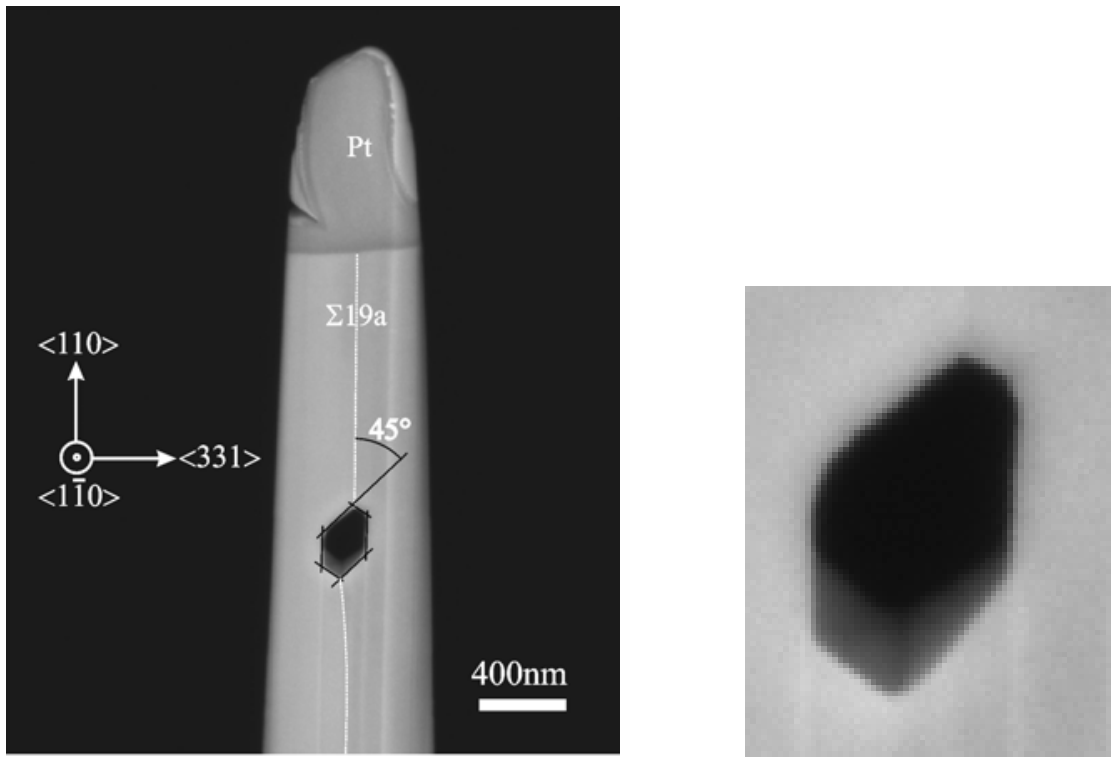

Abb. 4.39: Facettierter Lunker in einer bikristallinen Cu-Bi-Probe; links: Übersichtsbild mit Korngrenzverlauf; rechts: Ausschnittsvergrößerung des Lunkers

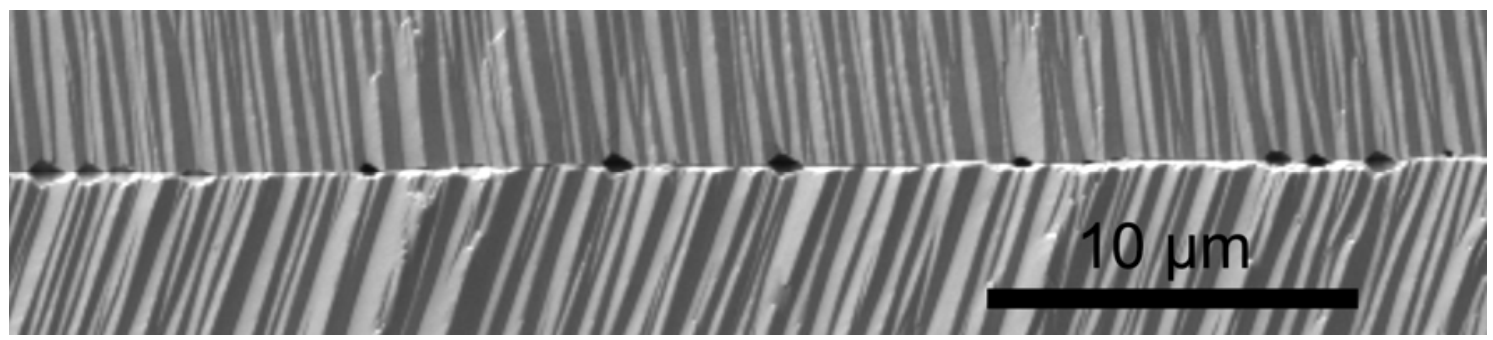

Abb. 4.40: Übersicht über die Lunkeranordnung; Aufsicht in $<110>$-Richtung; die Streifenstruktur in den angrenzenden Körnern ist eine Folge des anisotropen Ätzens bei der Probenpräparation.

Die Versprödung der Korngrenzen ist im Cu-Bi-Legierungssystem ein bekannter Effekt. Der Übergang von duktilem zu sprödem Verhalten findet Im Fall der $519 a$ Korngrenze zwischen 40 und $120 \mathrm{ppm}$ Bi statt. Nach Alchagirov et al. [AlCHAGIROv04] weist Bi eine Anomalie wie das Wasser auf und dehnt sich beim Erstarren aus. So kann auch ein abrupter Wechsel der Dichte der Segregationszone beim Erstarren als Ursache der Versprödung angenommen werden. Ob dies oder die Facettierung der Korngrenze ursächlich für die Versprödung sind, konnte mit den eingesetzten Methoden jedoch nicht geklärt werden. 


\section{Diskussion}

\subsection{Co-Evaporierte Ag-Ni Proben}

Es soll im Folgenden ein Bezug der experimentellen Ergebnisse der nanostrukturellen Entwicklung der Proben zur Theorie aufgestellt werden. Die beobachteten Unterschiede der Entwicklung der Proben mit hohem und niedrigem Ni-Gehalt erfahren zunächst eine getrennte Betrachtung. Die gewonnenen Erkenntnisse werden dann $\mathrm{zu}$ einem geschlossenen Bild zusammengefügt.

Zur Kompaktierung des kondensierten Materials wird ein Druck von $1 \mathrm{GPa}$ aufgebracht. Die dabei stattfindende starke Verformung senkt die minimale Temperatur für den Rekristallisationsbeginn deutlich herab. Aufgrund der gespeicherten Verformungsenergie wirkt auf die Korngrenzen eine treibende $\operatorname{Kraft} p$, die sich aus der Versetzungsdichte $\rho$ und dem Schubmodul $G=29,2$ GPa für die Ag-Matrix berechnet. Es gilt nach [GOTTSTEIN01]

$$
p=\frac{1}{2} \rho G b^{2}
$$

wobei der Beitrag dieser Kraft jedoch als gering eingeschätzt werden kann, da die Körner in Pressrichtung Ausdehnungen zwischen 2 und $7 \mathrm{~nm}$ haben. Versetzungen erzeugen ein weitreichendes Spannungsfeld, welches mit $1 / r$ abfällt. Es besteht somit eine attraktive bzw. repulsive Wechselwirkung von Versetzungen mit ungleichem bzw. gleichem Vorzeichen innerhalb eines Ag-Korns. Es ist anzunehmen, dass die Ag-Körner bei der starken Kaltverformung bereits dynamisch rekristallisiert sind und die verbliebenen Versetzungen sich in ihrer Gesamtheit als Großwinkelkorngrenzen angesammelt haben. Im Folgenden soll daher der Beitrag der Verformungsenergie zur Korngrenzenwanderung vernachlässigt werden. Bei der Beschreibung der ablaufenden Prozesse muss berücksichtigt werden, dass die Rekristallisationsvorgänge nicht in einem reinen Metall, sondern in einer zweiphasigen Legierung ablaufen. Kleine, fein verteilte Partikel einer solchen Phase behindern die Versetzungsbewegung und Korngrenzenwanderung stark. In einer Abwandlung des Orowan-Mechanismus der 
Ausscheidungshärtung kann die Behinderung der Rekristallisationsfront durch $\mathrm{Ni}$ Teilchen eines mittleren Abstandes beschrieben werden. Ein kugelförmiges Ni-Korn mit Radius $r_{0}$ befindet sich in der Matrix. Eine wandernde, ebene Korngrenze muss aufgrund ihrer Auswölbung eine zusätzliche Energie von

$$
\Delta E=4 \bar{\gamma} \pi r_{0}^{2}
$$

an Korngrenzen-Energie aufbringen, um dieses zu überwinden. Durch die Kenntnis des ausgeschiedenen Volumenbruchteils $v$ der zweiten Phase folgt aus geometrischen Gründen, dass

$$
n=\frac{3 v}{2 \pi r_{0}^{2}}
$$

Ausscheidungen pro Flächeneinheit der Korngrenze durchsetzt werden. Die auf die Korngrenze wirkende Haltekraft $p$ ist dann maximal

$$
p_{\max }=n \cdot \frac{\Delta E}{r_{0}}=\frac{6 v \bar{\gamma}}{r_{0}}
$$

Eine treibende Kraft diese Größe würde die Korngrenze zu einem Krümmungsradius von $R=2 \bar{\gamma} / p_{\max }$ auswölben, wobei $R$ unabhängig von der Korngrenzenergie ist. Der ausgeschiedene Volumenbruchteil kann als konstant angenommen werden. Damit können die beobachteten Korngrenzwölbungen in den Proben mit hohem Ni-Gehalt gut beschrieben werden. Für die beobachteten Ni-Korngrößen nach $10 \mathrm{~min}$ bei $200{ }^{\circ} \mathrm{C}$ ergibt sich danach ein maximaler Wölberadius zwischen den Ni-Körnern von $10 \mathrm{~nm}$ bis $25 \mathrm{~nm}$. Diese Daten stimmen gut mit den beobachteten Radien von $10 \mathrm{~nm}$ überein (Abb. 5.1a). Nach $30 \mathrm{~min}$ bei $400^{\circ} \mathrm{C}$ ist die Anzahldichte der Ni-Körner bereits soweit gefallen, dass viele TAP-Messungen nur noch Ag-Körner mit leicht gewölbten Korngrenzen zeigen. Es ist anzunehmen, dass diese im Begriff sind, sich bei längerer Auslagerungszeit wieder zu glätten. In Abwesenheit von Ni-Körnern finden sich typischerweise Wölberadien zwischen 25 und $50 \mathrm{~nm}$ (Abb. 5.1b). In den Proben mit niedrigem Ni-Gehalt wurden nur ungekrümmte Korngrenzen beobachtet. Dies ist mit 
der Annahme einer durch Ni-Körner behinderten Rekristallisationsfront konsistent. Da die Anzahldichten zu gering waren, um mit in TAP-Analysen detektiert zu werden, wurden auch nur in ihrer Bewegung ungestörte Ag-Korngrenzen beobachtet.

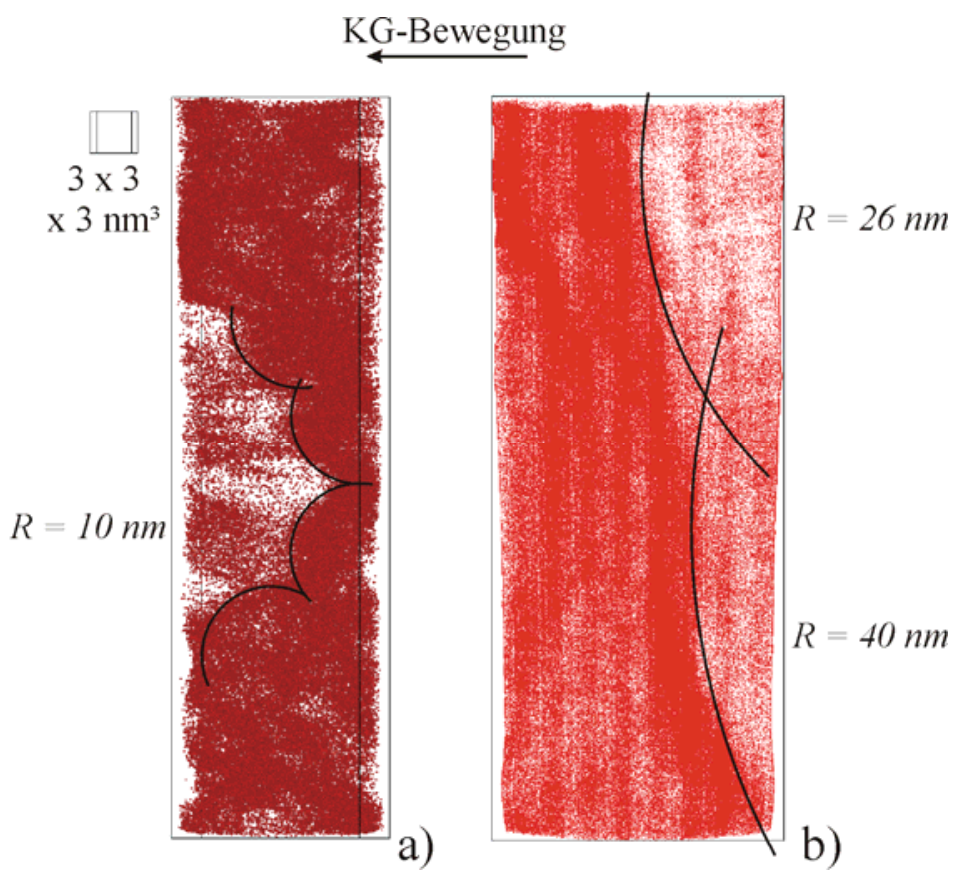

Abb. 5.1: Beobachtete KG-Wölberadien an ausgelagerten Proben mit hohem NiGehalt; a) nach $10 \mathrm{~min}$ bei $200{ }^{\circ} \mathrm{C}$; b) nach $30 \mathrm{~min}$ bei $400^{\circ} \mathrm{C}$

Aus der Beobachtung einer sehr niedrigen Anzahldichte der Ni-Körner im Zustand nach $30 \mathrm{~min}$ bei $400^{\circ} \mathrm{C}$ muss auf einen Umlösungsprozess geschlossen werden. Dies ist konsistent mit der Beobachtung glatter Korngrenzen in den Proben mit geringem NiGehalt, da diese bereits mit einer sehr niedrigen Anzahldichte starten. Da es sich jedoch um ein nicht mischbares Legierungssystem handelt, finden sich in der Literatur kaum thermodynamische Daten zur quantitativen Beschreibung dieses Prozesses. Dennoch kann die beobachtete Bildung von plattenförmigen Ni-Körnern in der Ag-Matrix beschrieben werden. Die Gitterfehlpassung zwischen $\mathrm{Ag}$ und $\mathrm{Ni}$ beträgt in $<100>$-Richtung $\delta=16,2 \%$. Aus diesem Grund muss eine Verzerrungsenergie $\Delta f_{\delta}$ berücksichtigt werden, die nach Haasen [HAASEN94] ausgedrückt werden kann als

$$
\Delta f_{\delta}=\frac{E \cdot \delta}{1-\mu} \cdot \varphi(c / b)
$$


wobei $E=83 \mathrm{GPa}$ der Elastizitätsmodul der Ag-Matrix und $\mu=0,37$ die Querkontraktionszahl der Matrix sind. $\varphi$ ist ein Formfaktor, der von dem Achsverhältnis $c / b$ des Ni-Korns abhängt (Abb. 5.2). Der Beitrag dieser Energie ist im Fall einer Kugel maximal.

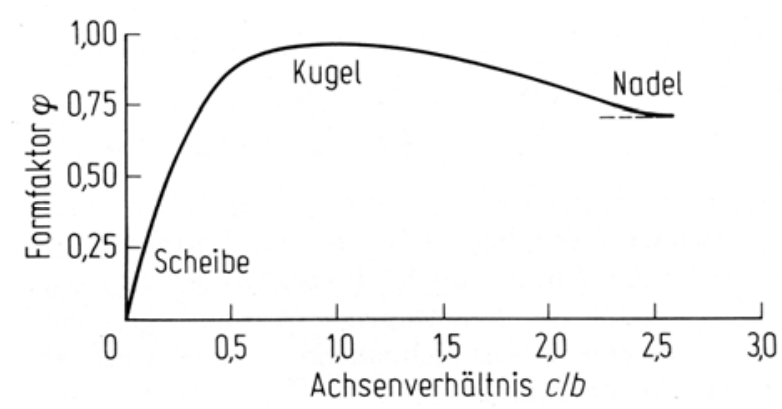

Abb. 5.2: Formfaktor der Verzerrungsenergie als Funktion des Achsverhältnisses einer ellipsoiden Ausscheidung

Eine deutliche Absenkung kann erzielt werden, indem die Ni-Körner ein niedriges Achsverhältnis einnehmen, was einer scheibenförmigen Morphologie entspricht. Die beobachteten Ni-Körner besitzen im Zustand nach 30 min bei $400{ }^{\circ} \mathrm{C}$ ein Achsverhältnis von etwa $c / b=4 \mathrm{~nm} / 37 \mathrm{~nm}$, wobei die Energieersparnis gegenüber der Kugelform groß ist. Entsprechende Werte wurden für eine Kugel mit $\varphi=1$ und eine Scheibe mit dem beobachteten Achsverhältnis von $\varphi=0,11$ berechnet und finden sich in Tabelle 5.1 .

\begin{tabular}{|l|c|c|c|}
\hline & $\boldsymbol{c} / \boldsymbol{b}$ & $\boldsymbol{\varphi}(\boldsymbol{c} / \mathbf{b})$ & $\boldsymbol{\Delta} \boldsymbol{f}_{\boldsymbol{\delta}}$ \\
\hline Kugel & 1 & 0,93 & $21,3 \mathrm{GJ} / \mathrm{m}^{3}$ \\
\hline Scheibe & 0,11 & 0,22 & $4,7 \mathrm{GJ} / \mathrm{m}^{3}$ \\
\hline
\end{tabular}

Tab. 5.1: Berechnete Werte der Verzerrungsenergie im Ag-Ni-Legierungssystem für ein kugelförmiges und ein scheibenförmiges Ni-Korn. 
Die nanostrukturelle Entwicklung kann daher folgendermaßen beschrieben werden:

Der Ausgangszustand besteht aus einem lamellaren Gefüge aus Ag-Körnern. Die ursprüngliche Pressrichtung liegt, aufgrund der gewählten Probenpräparation, senkrecht zur FIM/TAP-Analyserichtung. Die Ni-Körner sind statistisch über das Gefüge verteilt (Abb. 5.3a). Nach einer kurzen Auslagerung bei der Rekristallisationstemperatur von $\mathrm{Ag}$ von $200{ }^{\circ} \mathrm{C}$ wird bereits eine deutliche Bewegung der Ag-Korngrenzen beobachtet (Abb. 5.3b). Eine Stabilisierung des Gefüges durch eine Ni-Segregation in die Korngrenzen findet somit nicht statt. Die Ni-Körner behindern die Bewegung der AgKorngrenzen jedoch, die sich in guter Übereinstimmung mit dem vorgestellten Modell auswölben. Als schnelle Diffusionswege stehen den Ni-Atomen bei dieser Temperatur nur die Korngrenzen zur Verfügung. So entstehen bevorzugt an parallel zur Ausbreitungsrichtung der Korngrenzen angeordneten Tripellinien Ni-Ausscheidungen, die aufgrund der Verzerrungsenergie plattenförmig wachsen. Nach längerer Auslagerung werden nur noch Ag-Korngrenzen beobachtet, die leichte Wölbungen aufweisen. Diese haben Ni-Körner außerhalb des TAP-Analysevolumens bereits überwunden und glätten sich im Zuge des weitern, unbehinderten Ag-Kornwachstums wieder (Abb. 5.3c).
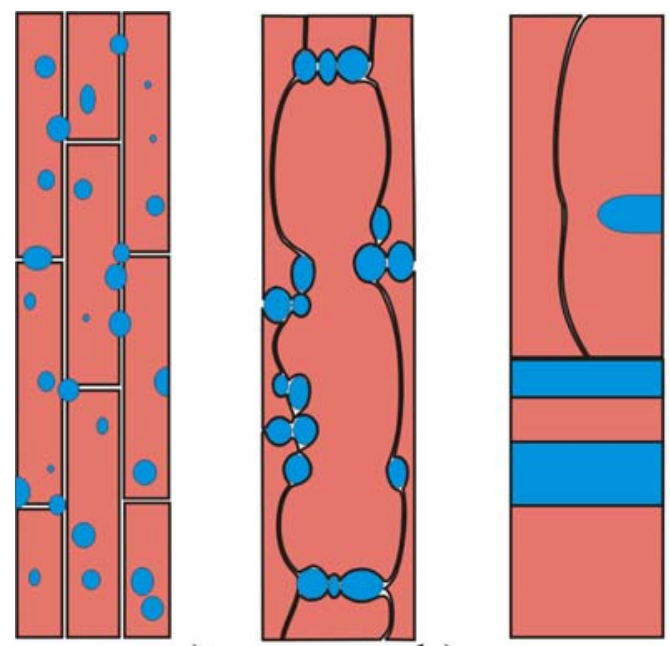

a)

b)

c)

Abb. 5.3: Schematische Entwicklung der Mikrostruktur in den Proben mit hohem NiGehalt; a) „as-prepared“-Zustand; b) nach $10 \mathrm{~min}$ bei $200{ }^{\circ} \mathrm{C}$; c) nach $30 \mathrm{~min}$ bei $400{ }^{\circ} \mathrm{C}$ 


\subsection{Ag-Ni Multilagen}

\subsubsection{Modell des Schichtzerfalls}

Es wurde im Zuge der Auslagerung keine Interreaktion der Schichten festgestellt. Die Grenzflächen wiesen in allen Zuständen keinerlei Durchmischung auf. Eine Segregation von $\mathrm{Ni}$ in die Ag-Korngrenzen wurde auch bei hohen Temperaturen bis $400{ }^{\circ} \mathrm{C}$ in keiner Analyse beobachtet. Lezzar et al. [LEZZAR04] simulierten unter Annahme eines modifizierten Finnis-Sinclair Potentials [FINNIS85] die Segregationsenergie in einer Ag dotierten $\mathrm{Ni}$ - und einer Ni-dotierten Ag-Legierung (im Folgenden als $\mathrm{Ni}(\mathrm{Ag})$ und $\mathrm{Ag}(\mathrm{Ni})$ bezeichnet). Simuliert wurden zwei unterschiedliche $\Sigma 11<110>$ Fälle mit den kohärenten Korngrenzen vom Typ $\{311\}$ und $\{233\}$. Erstere hat eine einfache atomare Struktur mit einer kleinen Korngrenzenergie, während letztere durch ihre offenere Struktur (,zick-zack“) eine etwa doppelt so hohe Korngrenzenergie aufweist [HARdouin00]. Es gelang durch Variation der Atomgrößen von Matrixatomen am Ort der Korngrenze eine Berechnung des „size effect“. Der Beitrag dieses Effektes dominiert im Fall des Ni(Ag)- bzw. Ag(Ni)-Legierungssystems die Gesamtenergie der Segregation. Interessanterweise korrespondieren die Orte starker Ag-Segregation in NiBikristallen mit denjenigen Atompositionen, die an der Korngrenze unter Zugspannungen stehen. Im umgekehrten Fall eines Ag-Bikristalls wird das Gegenteil beobachtet, die Segregationsenthalpie von $\mathrm{Ni}$ an diesen Orten der Korngrenzen ist positiv. Die Korngrenze verarmt an diesen Stellen an Ni. Eine Zusammenfassung dieser Ergebnisse findet sich in Abbildung 5.4. 


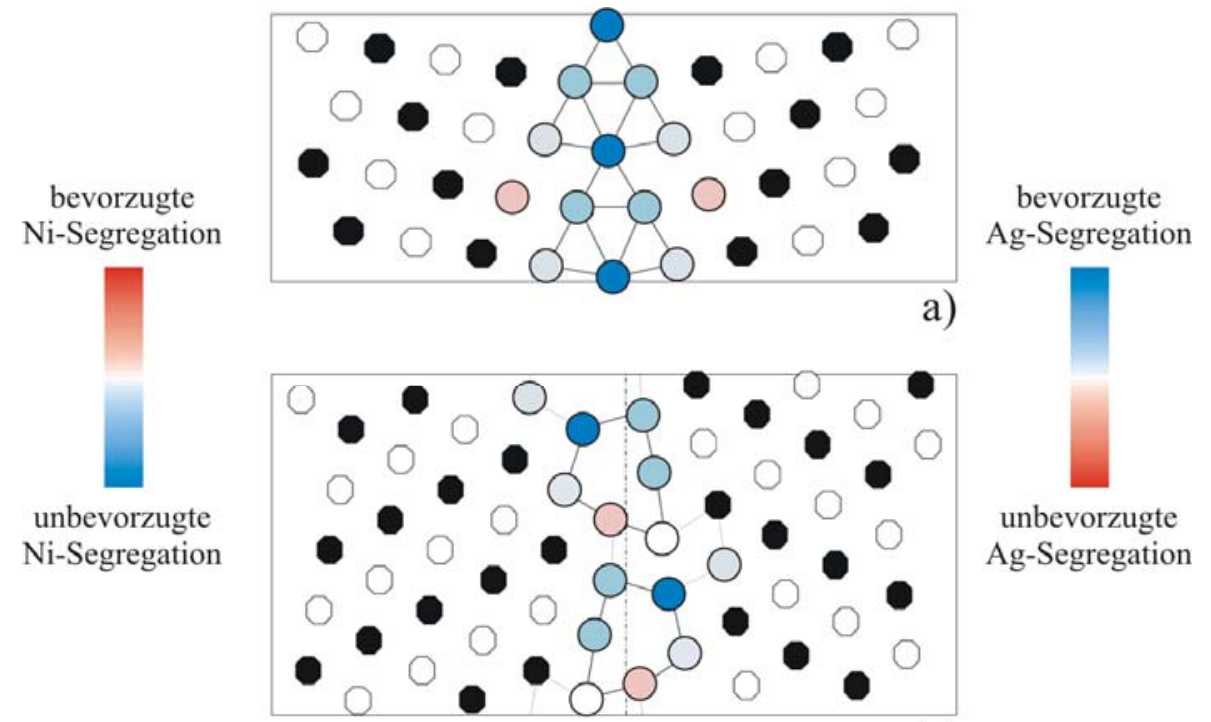

b)

Abb. 5.4: Zusammenfassung der Ergebnisse von Lezzar et al. [LEZZAR04] an 211 Korngrenzen in Ag- bzw. Ni-Bikristallen mit jeweiligen Struktureinheiten; a) mit (311)Korngrenze; b) mit (332)-Korngrenze

Es ist offensichtlich, dass eine Ni-Segregation bei den modellierten Strukturen mit niedriger und hoher Energie nur an sehr wenigen Korngrenzlagen leicht bevorzugt ist. Dieser Effekt wird somit auf den Größenunterschied der $\mathrm{Ag}$ und $\mathrm{Ni}$ Atome zurückgeführt. Die im Vergleich zu Ag kleineren Ni-Atome segregieren nicht in den Ag-Korngrenzen, da dort ein größeres Exzessvolumen vorliegt. Für $\mathrm{Bi}$ in $\mathrm{Cu}-$ Korngrenzen sind die Größenverhältnisse genau umgekehrt, was zu einer starken Segregation führt [SCHWEINFEST04]. Die Ergebnisse von Lezzar et al. entsprechen den experimentellen Ergebnissen dieser Arbeit sehr gut, da weder in den co-evaporierten noch in den sputterdeponierten Proben eine Korngrenzsegregation von Ni in die AgKorngrenzen beobachtet wurde. Die Ergebnisse der Simulationen an den 211 Korngrenzen können somit auf allgemeine Korngrenzen in Ag übertragen werden. Die fehlende Segregation von $\mathrm{Ni}$ in Ag-Korngrenzen ist auch ein überzeugendes Gegenbeispiel für die von Hondros und Seah propagierte These, dass eine geringe Löslichkeit zu einer starken Korngrenzsegregation führt [HONDROS96]. Unter der Annahme einer bevorzugten Segreagtion im umgekehrten Legierungssystem können auch die in den Messungen der wärmebehandelten, co-evaporierten Proben beobachteten Stege zwischen den Ni-Körnern aus reinem Ag erklärt werden. Deren 
Dicke beträgt einige Monolagen nach $10 \mathrm{~min}$ bei $200^{\circ} \mathrm{C}$. Möglicherweise schafft sich die Mikrostruktur bevorzugt Ni-Korngrenzen, in denen Ag-Atome segregieren. Neueste Ergebnisse von Eickemeyer et. al [EICKEMEYER05] zeigen bereits beim Zulegieren von $0,01 \mathrm{At} . \% \mathrm{Ag}$ in $\mathrm{Ni}$ nach dem Heißwalzen und Rekristallisieren deutlich kleinere Korngrößen als im reinen Ni. Hinzu kommt eine Korngrenzstruktur, die kaum gerade Segmente aufweist, sondern versucht, durch viele Krümmungen ein Maximum an Korngrenzfläche zu schaffen (Abb. 5.5). Die Absenkung der Korngrenzenergie durch eine starke Ag-Segregation wird als ursächlich für dieses Verhalten angesehen. Auch diese Ergebnisse unterstützen die Simulationen von Lezzar et al. und zeigen die Möglichkeit deren Übertragung auf allgemeine Korngrenzen in Ni auf.

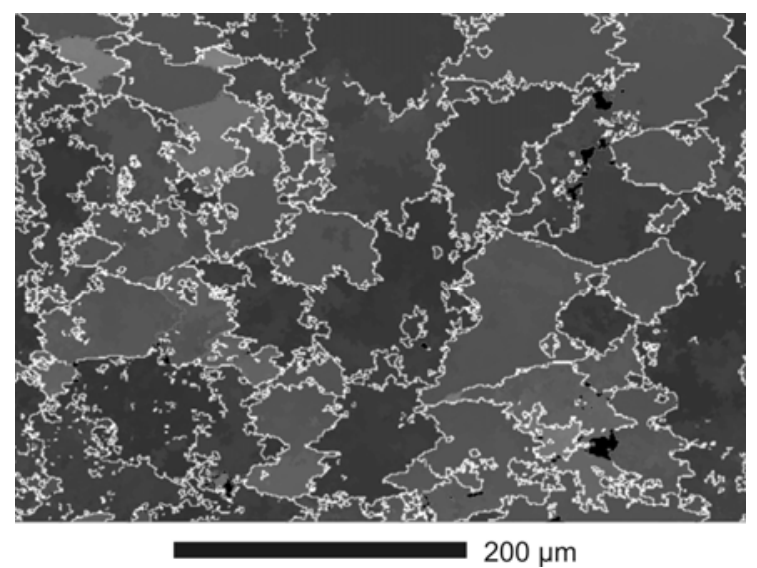

Abb. 5.5: Korngrenzen (weiß) einer heißgewalzten Ni-0,01 At. \% Ag Legierung nach einer Rekrstallisationsbehandlung von $30 \mathrm{~min}$ bei $550{ }^{\circ} \mathrm{C}$ nach Eickemeyer et al. [EICKEMEYER05]

Somit können nun auch die anfangs vorgestellten Beobachtungen von Schweitz et al. [SCHWEITZ01] (Abschnitt 1.2.1, Abb. 1.6) gedeutet werden. Die Ag-Brückenbildung über die Ni-Schichten ist einer Segregation in die Ni-Korngrenzen zuzuschreiben und bewirkt den Zerfall des GMR-Modellsystems. Eine gezielte Untersuchung des umgekehrten Legierungssystems Ni-Ag erscheint im Lichte neuester Erkenntnisse für weitere Forschungen interessant. Die in dieser Arbeit weiterentwickelte „Lift-Out“ Präparation bietet sich insbesondere zur Untersuchung der Korngrenzen der heißgewalzten $\mathrm{Ni}-0,01$ At. \% Ag Legierung sehr gut an und könnte die postulierte Segregation von Ag verifizieren und quantifizieren. 


\subsubsection{Mittlerer Oberflächendiffusionskoeffizient}

Eine Interreaktion zwischen den Ag- und Ni-Multilagen wurde nicht beobachtet. Stattdessen wurde die hergestellte Schichtstruktur bei Wärmebehandlungen $>1 \mathrm{~h}$ bei $400{ }^{\circ} \mathrm{C}$ zerstört, indem sich die Schichten zum Schaftbereich der Spitze zurückgezogen haben. Nach einem Modell von Barbour et al. [BARBOUR60] kann der Oberflächendiffusionskoeffizient aus der Beobachtung der Abbaugeschwindigkeit der Spitze in $z$-Richtung ermittelt werden (Abb. 5.6).

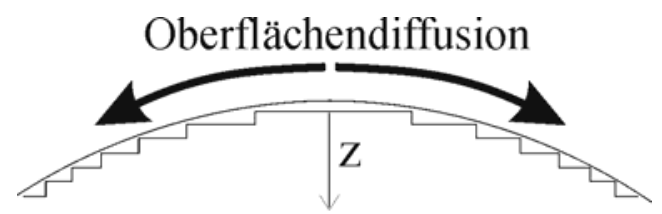

Abb. 5.6: Schematische Darstellung einer FIM-Spitze

An einer gekrümmten Oberfläche gilt für das chemische Potential $\mu=K \sigma \Omega$, wobei $\mathrm{K}$ die Krümmung, $\sigma$ die Oberflächenenergie und $\Omega$ das Atomvolumen ist. Die Driftgeschwindigkeit von Oberflächenatomen folgt nach der Nernst-Einstein-Relation aus dem Produkt der Beweglichkeit und der Kraft $v=B \cdot F$, mit $B=D / k T$ und $F=-d \mu / d s$. Dabei ist $D_{s}$ der Oberflächendiffusionskoeffizient und $s$ die Bogenlänge entlang des Spitzenprofils.

$$
v=\frac{D_{s}}{k T} \cdot \frac{\partial \mu}{\partial s}=\frac{D_{s} \cdot \Omega \cdot \sigma}{k T} \cdot \frac{\partial K}{\partial s}
$$

Der Teilchenstrom über die Oberfläche ist das Produkt der Geschwindigkeit $v$ und der Zahl von Atomen pro Einheitsfläche $N_{s}$ :

$$
j=v \cdot N_{s}=\frac{D_{s} \cdot \Omega \cdot N_{s} \cdot \sigma}{k T} \cdot \frac{\partial K}{\partial s}
$$


Die Änderung der Zahl der Atome pro Einheitsfläche und Zeit ist somit

$$
\operatorname{div} j=\frac{D_{s} \cdot \Omega \cdot N_{s} \cdot \sigma}{k T} \cdot \frac{\partial^{2} K}{\partial s^{2}}
$$

Heumann modellierte die Krümmung an typischen FIM/TAP-Spitzen [HEUMANN92] und erhielt $\partial^{2} K / \partial s^{2}=1,25 / r^{3}$. Die Multiplikation mit dem Atomvolumen führt dann auf die Abbaugeschwindigkeit senkrecht zur Oberfläche:

$$
\frac{d z}{d t}=\left(\frac{1.25}{r^{3}}\right) \cdot\left(\frac{\Omega^{2} \cdot N_{s} \cdot \sigma}{k T}\right) \cdot D_{s} .
$$

Für die auf Spitzen deponierten Ag-Ni-Schichten kann im „as-sputtered“-Zustand in guter Übereinstimmung mit TEM-Untersuchungen ein Krümmungsradius von $30 \mathrm{~nm}$ angenommen werden. Der Einfluss der Ni-Schichten kann für die folgende Betrachtung aufgrund ihrer Dicke von nur wenigen [111]-Monolagen vernachlässigt werden. In FIM-Analysen wird eine starke $<111>$-Epitaxie der Schichten beobachtet. Daher wird zur Berechnung der Abbaugeschwindigkeit $d z / d t$ die Oberflächenenergie $\sigma_{s}=1282 \mathrm{~mJ} / \mathrm{m}^{2}$ [WAN99] einer Ag (111)-Ebene mit 13,81 Atome $/ \mathrm{nm}^{2}$ verwendet. Mit dem Atomvolumen $\Omega_{A g}=17,056 \AA^{3}$ ergibt sich durch Integration von Gleichung 5.9 der nach der Auslagerungszeit zurückgelegte Weg bei der Glühtemperatur. Als Parameter steht dabei der Oberflächendiffusionskoeffizient $D_{s}$ zur Verfügung. Abbildung 5.7 zeigt das Ergebnis der Integration nach $1 \mathrm{~h}$ bei $400^{\circ} \mathrm{C}$ in Abhängigkeit von $D_{s}$ und den direkten Vergleich mit einer zurückgezogenen Schicht. Die Schichten waren typischerweise um Längen zwischen 100 - $140 \mathrm{~nm}$ zurückgezogen. Daraus folgt ein Oberflächendiffusionskoeffizient von $D_{s}=2.3-3 \cdot 10^{-15} \mathrm{~m}^{2} / \mathrm{s}$. Aufgrund des Fehlers bei der Bestimmung von $z$ kann hier kein exakter Wert angegeben werden. Die Oberflächendiffusion kann um mehrere Größenordnungen schneller ablaufen als die Volumendiffusion, die bei dieser Temperatur einen Diffusionskoeffizienten von $D_{\text {Vol, } A g}=2,73 \cdot 10^{-19} \mathrm{~m}^{2} / \mathrm{s}$ aufweist [LANDOLT90]. Dies entspricht qualitativ dem experimentellen Befund. 


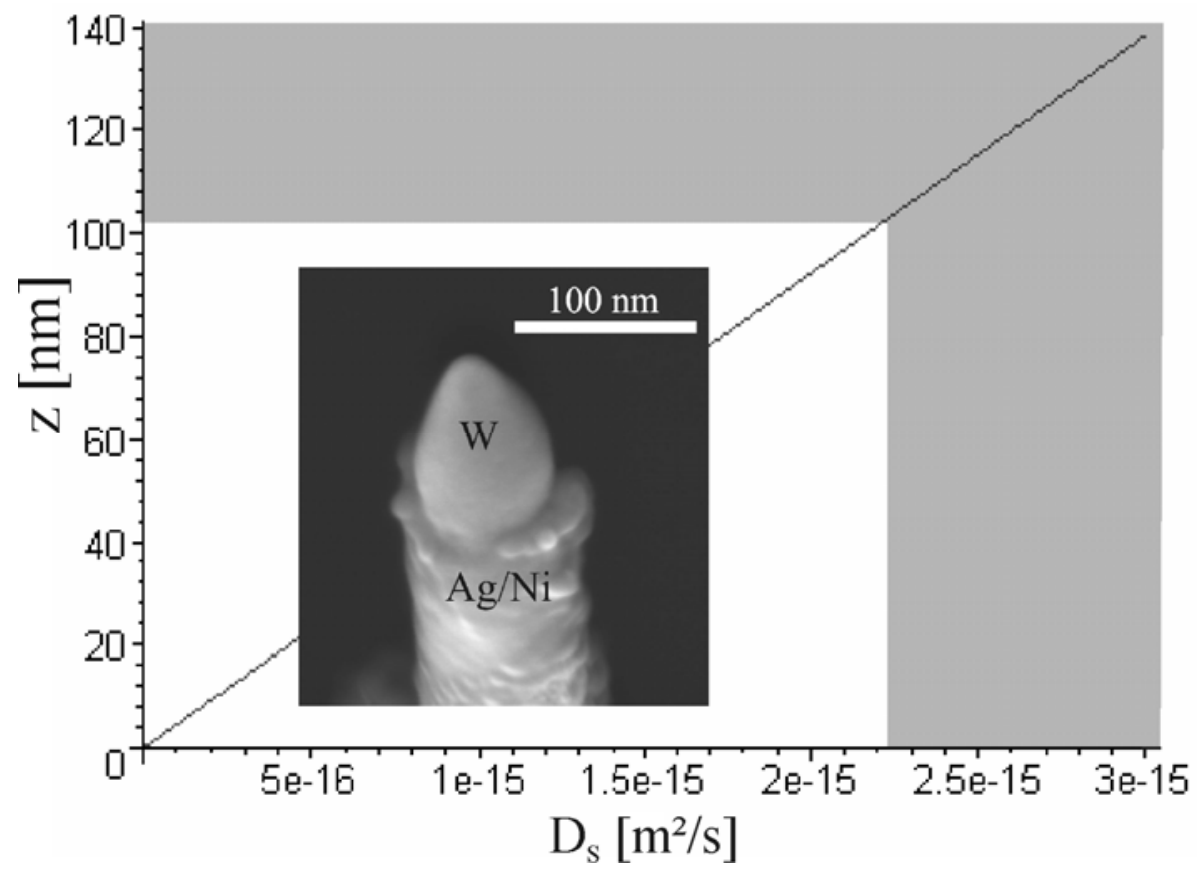

Abb. 5.7: Abhängigkeit der Abbaulänge der Spitze in z-Richtung vom Oberflächendiffusionskoeffizienten

Der Abbau der Schichten erfolgt wie in Abbildung 5.8 schematisch dargestellt. Dieses Modell liefert eine Erklärung für den mit der Auslagerungsdauer und Temperatur zunehmenden Verlust von Schichten. Die ideale Geometrie der Spitze wird zerstört.

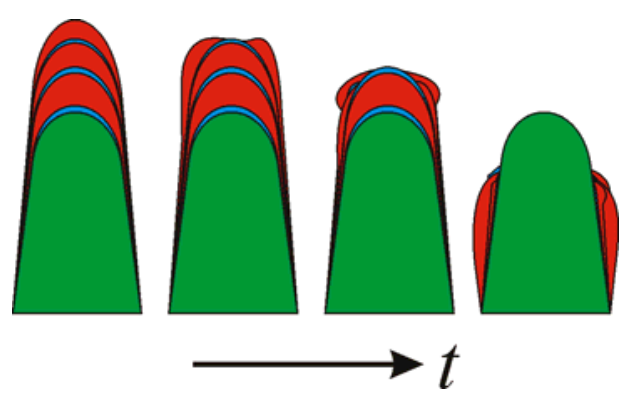

Abb. 5.8: Zerfall der Ag-Ni-Schichten bei $400^{\circ} \mathrm{C}$ (schematisch) 


\subsection{Bi dotierte 219 a Cu-Bikristalle}

Als Grundlage wurde für die Untersuchung des Cu-Bi-Systems eine symmetrische $\Sigma 19 a$ Korngrenze gewählt. Die Belegungsdichte wurde unter Zuhilfenahme des Programms „CaRIne Crystallography“ [CARINE98] modelliert. Einer \{331\}-Monolage entsprechen 7,2 Cu-Atome $/ \mathrm{nm}^{2}$. Die zugehörige Korngrenzenergie wurde von Schweinfest et al. [SCHWEINFEST04] durch quantenmechanische ab initio Berechnungen für reines $\mathrm{Cu}$ zu $\gamma_{0}=1040 \mathrm{~mJ} / \mathrm{m}^{2}$ bestimmt. Diese Korngrenze stellt eine erhebliche Störung des Kristalls dar, wie der Vergleich mit der Oberflächenenergie von reinem $\mathrm{Cu}$ mit $\sigma_{C u}=1780 \mathrm{~mJ} / \mathrm{m}^{2}$ zeigt [TYSON77], [HIRTH68]. Im Folgenden sollen aus den in dieser Arbeit gemessenen Exzesswerten und Literaturdaten die Segregationsenthalpie und die Absenkung der Korngrenzenergie ermittelt und diskutiert werden. Aufgrund der definierten Randbedingungen sind die Ergebnisse letztlich als zuverlässig einzustufen.

\subsubsection{Absenkung der Korngrenzenergie}

Aus der Messung der Exzesswerte einer Messreihe bei konstanter Temperatur und variablen Dotierungen im Bereich der Gleichgewichtslöslichkeit kann über die Gibbssche Adsorptionsisotherme auf die Absenkung der Korngrenzenergie durch die Bi-Segregation geschlossen werden. Da es im Bereich weniger ppm experimentell überaus schwierig ist, solche Dotierungen einzustellen und den Exzesswert an der Korngrenze durch Messungen quantitativ zu verifizieren, wurden solche Messreihen bisher nicht gezielt durchgeführt. Sigle et al. [SIGLE02] untersuchten ebenfalls einen mit Bi dotierten $519 a$ Bikristall mittels EDX an einem STEM. Sie beobachteten eine Facettierung der Korngrenze mit unterschiedlichen Bi-Exzessen an den Facetten (Tab. 5.1). Diese Werte sind aufgrund der identischen Probenpräparation der Bikristalle gut für einen Vergleich mit dem durch TAP bestimmten Exzess geeignet. Eine thermodynamische Betrachtung dieser Ergebnisse sowie ein Vergleich mit den Ergebnissen dieser Arbeit sollen im Folgenden durchgeführt werden. 


\begin{tabular}{|l|c|c|c|c|}
\hline $\boldsymbol{T}=\mathbf{8 0 0}^{\circ} \mathbf{C}$ & $\begin{array}{c}\boldsymbol{\Gamma}_{\mathbf{B} \boldsymbol{i}} \text { nicht facet. } \\
{\left[\text { Atome } / \mathrm{nm}^{2}\right]}\end{array}$ & $\begin{array}{c}\boldsymbol{\Gamma}_{\boldsymbol{B} \boldsymbol{i}} \text { facet. } \\
{\left[\text { Atome } / \mathrm{nm}^{2}\right]}\end{array}$ & facet. Anteil & $\begin{array}{c}\text { mittl. Exzess } \\
{\left[\text { Atome } / \mathrm{nm}^{2}\right]}\end{array}$ \\
\hline $25 \mathrm{ppm}$ & $8,4 \pm 1,8$ & $12,5 \pm 5$ & $38 \%$ & 10 \\
\hline $64 \mathrm{ppm}$ & - & $21 \pm 4$ & $100 \%$ & 21 \\
\hline
\end{tabular}

Tab. 5.1: Exzesswerte an einer 519 a nach einer Segregationsbehandlung bei $800{ }^{\circ} \mathrm{C}$ von Sigle et. al [SIGLE02]

Der Exzess wird für die weitere Betrachtung über die facettierten und nicht facettierten Bereiche gemittelt. Eine Umformung und Integration der Gleichung (1.14) ergibt

$$
\Delta \gamma_{K G}=-\int_{c_{1}}^{c_{2}} \Gamma_{B i} \cdot R T d \ln c_{B i}=-\left\langle\Gamma_{B i}\right\rangle \cdot R T \cdot \ln \frac{c_{1}}{c_{2}}
$$

Daraus folgt eine Absenkung der KG-Energie von 25 auf $64 \mathrm{ppm}$ um $216 \mathrm{~mJ} / \mathrm{m}^{2}$. Der mit TAP beobachtete Exzesswert liegt mit 3,2 $\pm 0,5 \mathrm{~nm}^{-2}$ bzw. 0,44 ML bei der Dotierung von $40 \pm 4 \mathrm{ppm}$ Bi unter dem von Sigle et al. gemessenen Wert für die mit 25 ppm dotierte Probe (Abb. 5.8). Bei Berücksichtigung dieses Wertes ergibt sich eine Absenkung der Korngrenzenergie um $190 \mathrm{~mJ} / \mathrm{m}^{2}$.

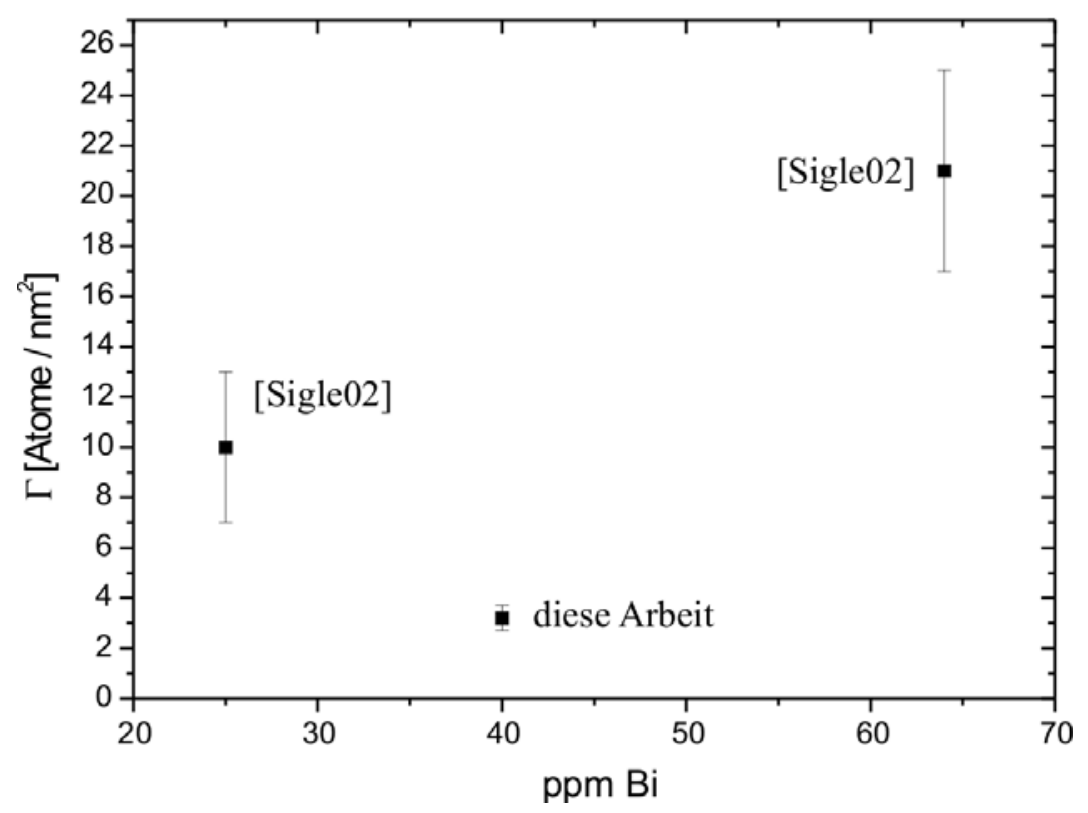

Abb. 5.8: Vergleich der Bi-Exzesse an einer 519 a Korngrenze; die Daten dieser Arbeit wurden bei $850^{\circ} \mathrm{C}$ aufgenommen, die von Sigle et al. bei $800^{\circ} \mathrm{C}$ 
Die Abweichung des Wertes bei 40 ppm kann durch die verschiedenen Temperaturen erklärt werden. Da das Phasendiagramm in diesem Bereich eine Änderung der Löslichkeit um etwa einen Faktor zwei zeigt, ist der Gewinn an Segregationsenthalpie bei einer Temperatur von $850^{\circ} \mathrm{C}$ kleiner als bei $800^{\circ} \mathrm{C}$. Daraus resultiert ein kleinerer Exzesswert. Dieser Trend wurde durch die Messungen bestätigt. Eine Absenkung der Korngrenzenenergie auf Null bzw. auf negative Werte kann bei Temperaturen von $800{ }^{\circ} \mathrm{C}-850^{\circ} \mathrm{C}$ im Konzentrationsbereich von $25-64 \mathrm{ppm}$ nicht bestätigt werden.

Die Segregationsenthalpie kann mit der Langmuir-Mclean-Gleichung aus dem beobachteten Exzess $\Gamma_{B i}$ und der maximalen Belegungsdichte $\Gamma_{B i \text { max }}$ der $\Sigma 19 a$ Korngrenze berechnet werden. Für extrem verdünnte Legierungssysteme gilt

$$
\Delta H_{\text {seg }}=-R T \ln \left(\frac{1}{c_{B i}} \cdot \frac{\Gamma_{B i}}{\Gamma_{B i, \text { max }}-\Gamma_{B i}}\right)=-93 \mathrm{~kJ} / \mathrm{mol}
$$

wobei aufgrund nur einer einzelnen Korngrenze in den beiden Bikristallhälften auch nach der Segregation in guter Näherung als Restlöslichkeit $c_{B i}$ die jeweilige Dotierung angenommen werden kann. Die Segregationsenthalpie muss zusätzlich um den Betrag einer anziehenden Wechselwirkungsenergie der Bi-Atome untereinander korrigiert werden. Dies wird durch eine Erweiterung von Gleichung 5.11 durch Fowler und Guggenheim berücksichtigt [FOWLER39]. Die um die Wechselwirkungsenergie korrigierte Segregationsenthalpie ist dann:

$$
\Delta H_{\text {seg }}^{0}=\Delta H_{\text {seg }}+\omega \cdot Z \cdot \frac{\Gamma_{B i}}{\Gamma_{B i, \max }}
$$

Die Wechselwirkungsenergie von $\mathrm{Bi}$ in stark gestörten $\mathrm{Cu}$-Korngrenzen wurde von Alber et al. mit - $43 \mathrm{~kJ} / \mathrm{mol}$ angegeben [ALBER99]. In der $519 a$ Korngrenze existieren maximal $Z=4$ nächste Nachbarn. Die korrigierte Segregationsenthalpie ist dann $\Delta H_{\text {seg }}^{0}=-17 \mathrm{~kJ} / \mathrm{mol}$. Der Vergleich mit Diffusionsexperimenten von Divinski et al. [DIVINSKI04] zeigt eine gute Übereinstimmung mit dem dort angegebenen Wert von - $29 \mathrm{~kJ} / \mathrm{mol}$ bei $1000 \mathrm{~K}$. Aufgrund der etwa um einen Faktor zwei geringeren 
Löslichkeit von $\mathrm{Bi}$ in $\mathrm{Cu}$ bei $1000 \mathrm{~K}$ und $1123 \mathrm{~K}$ ist der in dieser Arbeit ermittelte Wert wie erwartet kleiner.

\subsubsection{Segregation an Kleinwinkel-Korngrenzen}

In den gesputterten $\mathrm{Cu}(\mathrm{Bi})$-Schichten konnten bereits im unbehandelten Zustand Korngrenzen mit Bi-Anreicherungen detektiert werden. Diese wurden als KleinwinkelKippkorngrenzen mit Kippwinkeln von $\Theta \sim 12^{\circ}$ identifiziert. Der Abstand der aus zwei um einen Winkel $\Theta$ gegeneinander verkippten Körnern in die Korngrenze laufenden Versetzungen ergibt sich aus $h=b / \Theta=1,22 \mathrm{~nm}$ [KITTEL02].

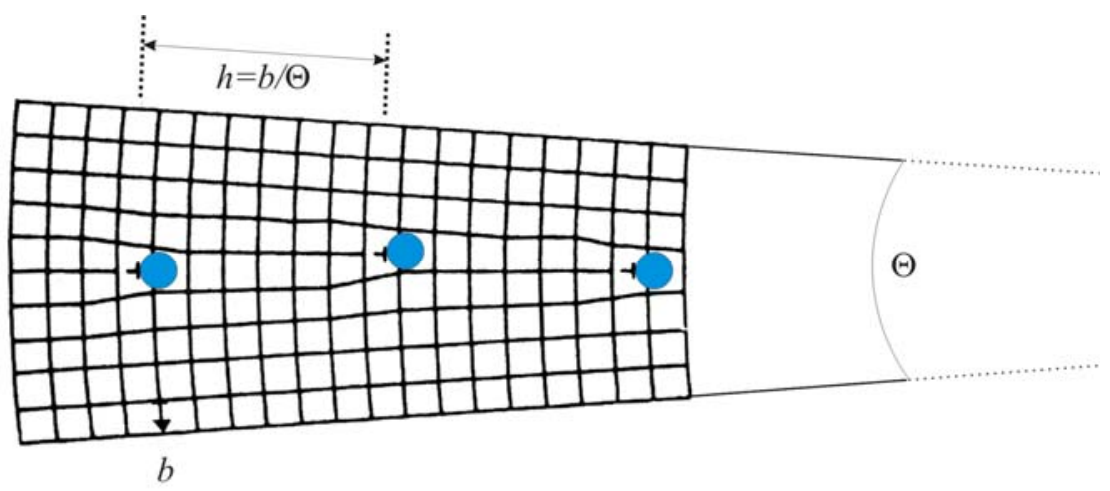

Abb. 5.9: Versetzungsstruktur an einer symmetrischen Kleinwinkel-Korngrenze; im Dilatationsfeld von Versetzungen segregieren bevorzugt Bi-Atome (blau).

Die Bi-Atome segregieren, bedingt durch ihre Größe, bevorzugt an Orten mit einer möglichst offenen Struktur. Insbesondere im Dilatationsfeld von Versetzungen herrscht eine solche Struktur vor. Eine mögliche Anordnung von Bi-Atomen ist in Abbildung 5.9 dargestellt. Geht man von Ketten von Bi-Atomen entlang der Versetzungen aus, so findet sich maximal ein Bi-Atom pro $h \cdot 2 \cdot r$ Fläche, wobei $r=0,204 \mathrm{~nm}$ der Atomradius von $\mathrm{Bi}$ ist. Der maximale Exzess einer solchen Konfiguration wäre im Fall der beobachteten Kleinwinkel-Korngrenzen daher $\Gamma_{K W, 12^{\circ}}=2,0$ Atome $/ \mathrm{nm}^{2}$. Dies entspricht dem gemessenen Wert von 1,3 Atomen $/ \mathrm{nm}^{2}$ gut und bestätigt die Anwendbarkeit dieses Konzeptes. Es sei an dieser Stelle nochmals darauf hingewiesen sein, dass die Korngrenzen bereits im ,as-sputtered“-Zustand diese 
Segregation aufwiesen. Eine vollständige Sättigung mit einem größeren Exzesswert wird möglicherweise erst nach einer Temperaturbehandlung erreicht.

\subsubsection{Lunkerstabilisierung durch Oberflächensegregation}

Die Größe der beobachteten Lunker wurde mit $320 \pm 90 \mathrm{~nm}$ bei einer mittleren Anzahldichte von 3,3 $10^{9} \pm 0,7 \cdot 10^{9}$ Lunker $/ \mathrm{m}^{2}$ bestimmt (Abb. 4.39 und 4.40). Die Anwesenheit von Lunkern ist zunächst unverständlich, da ein reiner Cu-Bikristall bei den tagelangen thermischen Behandlungen bei Temperaturen von bis $\mathrm{zu} 950^{\circ} \mathrm{C}$ vollständig sintern sollte. Da dies nicht der Fall ist, muss von einer bevorzugten Leerstellendrift zur Korngrenze ausgegangen werden, die dort zu einer Bildung von Poren führen kann. Wie López et al. [LÓPEZ03] ebenfalls an einer $\Sigma 19 a$ Korngrenze mit künstlich eingebrachten Kavitäten mit Ausdehnungen von einigen $10 \mu \mathrm{m}$ zeigten, ist die Segregation bei Temperaturen von $800-900^{\circ} \mathrm{C}$ an den freien Oberflächen wesentlich stärker als die Korngrenzsegregation. Mit diesem interessanten Befund kann die in dieser Arbeit beobachtete Porenbildung an der Korngrenze erklärt werden. Die freien Oberflächen der Poren werden durch eine bevorzugte Bi-Segregation stabilisiert und wachsen. Bereits bei der Dotierung bei $880^{\circ} \mathrm{C}$ wird $\mathrm{Bi}$ aus der Quelle in der Quarzampulle bis zur Sättigung nachgeliefert. Innerhalb eines Lunkers wachsen dann diejenigen Facetten, die die stärkste Bi-Segregation zulassen. Eine chemische Analyse des Lunkers und seiner Facetten mit dem TAP konnte nicht durchgeführt werden, da die Methode an solchen Defekten, die wesentlich größer als der Spitzenapex sind, vollständig versagt. 


\section{Zusammenfassung}

Die Korngrenzsegregation an ausgesuchten binären metallischen Systemen wurde im Rahmen dieser Dissertation mit hochauflösenden Methoden atomar untersucht. Es wurden mittels gemeinsamer thermischer Verdampfung bulk-nanokristalline Ag-NiLegierungen hergestellt und untersucht. Die Kombination von FIM- und TAP-Analysen liefert ein vollständiges Bild der Kornstruktur. Trotz einer hohen Porosität gelang es erstmals, solche Proben mit den eingesetzten Methoden zu untersuchen. Fein verteilte, sphärische Ni-Körner in einem lamellaren Gefüge von Ag-Körnern bilden den Ausgangszustand nach der Herstellung. Bereits nach einer Wärmebehandlung von $200^{\circ} \mathrm{C}$ findet nach $10 \mathrm{~min}$ ein Kornwachstum statt, welches durch die Anwesenheit der Ni-Körner behindert wird. Die wandernden Ag-Korngrenzen wölben sich mit Krümmungsradien, die durch einen abgewandelten Orowan-Mechanismus beschrieben werden können. Eine Segregation von Ni in die Ag-Korngrenzen wird nicht beobachtet. Die Stabilisierung des Gefüges durch Ni-Körner ist, bedingt durch deren schnelle Vergröberung, gering. Die ausgeprägte Gitterfehlpassung von 16,2\% von $\mathrm{Ag}$ und $\mathrm{Ni}$ erfordert die Berücksichtigung der Verzerrungsenergie, deren Beitrag zu einem plattenförmigen Wachstum der Ni-Körner führt.

Zur weiteren Untersuchung der Korngrenzsegregation wurden Ag-Ni-Multilagen mittels Ar-Ionen-Sputtern als dünne Schichten auf W-Substratspitzen deponiert. Eine epitaktisches Aufwachsen vom <110>-texturierten W-Substrat $\mathrm{zu}<111>$-texturierten $\mathrm{Ag}$ und Ni Schichten konnte nachgewiesen werden. Die Grenzflächen zeigten nach allen thermischen Behandlungen keinerlei Durchmischung. Durch TAP-Analysen konnten Ag-Korngrenzen zwischen den Ni-Schichten auf atomarer Skala aufgelöst werden und die erwartete kolumnare Struktur bestätigt werden. Entlang dieser konnte in keinem Zustand eine Segregation oder Diffusion von Ni entlang der Ag-Korngrenzen festgestellt werden. Die häufig propagierte These, dass eine geringe Löslichkeit einer Legierungskomponente $\mathrm{zu}$ einer starken Korngrenzsegregation führt, konnte somit durch ein überzeugendes Gegenbeispiel widerlegt werden. Der zunehmende Verlust von Schichten mit steigender Auslagerungstemperatur und Zeit konnte durch TEM- und REM-Analysen auf eine Oberflächendiffusion zurückgeführt werden, wobei der zugehörige Diffusionskoeffizient bei $400{ }^{\circ} \mathrm{C}$ etwa $3 \cdot 10^{-15} \mathrm{~m}^{2} / \mathrm{s}$ beträgt. 
Das Segregationsverhalten am thermodynamisch vergleichbaren $\mathrm{Cu}-\mathrm{Bi}$ Legierungssystem wurde zunächst an nanokristallinen Legierungsschichten untersucht, die ebenfalls durch Ar-Ionen-Sputtern hergestellt wurden. Zu diesem Zweck wurden zwei Komposit-Targets und ein Legierungs-Target erprobt. Aus Gründen der Stabilität der Schicht wurde Co als Haftvermittler eingesetzt. Durch FIM-Analysen konnte für diese $\mathrm{Cu}(\mathrm{Bi})$-Schichten ebenfalls ein kolumnares Wachstum bestätigt werden. TAPAnalysen zeigten, dass die Zusammensetzungen der Schichten auch in Proben derselben Charge Konzentrationsunterschiede von $0-1,5$ At. \% aufwiesen. Durch TAP-Analysen konnte bereits im ,as-sputtered“ Zustand eine Segregation von 1,3 Atomen $/ \mathrm{nm}^{2}$ an Korngrenzen nachgewiesen werden. Dieser Exzess konnte für die beobachteten Kleinwinkel-Kippkorngrenzen auf ein einfaches Modell der Versetzungsstruktur zurückgeführt werden. Aufgrund starker Versprödungserscheinungen war die Analyse thermisch behandelter Zustände nicht möglich. Die weiteren Untersuchungen wurden daher an bikristallinen $\mathrm{Cu}-40 \mathrm{ppm} \mathrm{Bi}$ durchgeführt, die im Gegensatz zu denen mit $120 \mathrm{ppm} \mathrm{Bi}$ einen duktilen Charakter aufwiesen. Als Korngrenze wurde eine $\Sigma 19 a\{331\}$ gewählt, deren spezifische Korngrenzenergie mit $1040 \mathrm{~mJ} / \mathrm{m}^{2}$ sehr hoch ist und somit eine starke Bi-Segregation erwartet werden kann.

Zur Untersuchung einer einzelnen, wohldefinierten Korngrenze musste eine neue Methode der Probenpräparation entwickelt werden. Mit Hilfe der vorgestellten „LiftOut"-Methode konnte erstmals die TAP-Analyse einer diskreten Korngrenze durchgeführt werden. Die Feldverteilungen um solche Proben wurden berechnet, wodurch im Rückschluss die Probengeometrie deutlich verbessert werden konnte. Aus den TAP-Analysen folgt bei einer Belegungsdichte der Korngrenze von 7,2 Atome $/ \mathrm{nm}^{2}$ ein Bi-Korngrenzenexzess von 0,44 ML, woraus aus thermodynamischen Betrachtungen eine um die Wechselwirkungsenergie korrigierte Segregationsenthalpie von $-17 \mathrm{~kJ} / \mathrm{mol}$ berechnet werden kann. Die Absenkung der Korngrenzenergie von 25 bis 64 ppm auf Null bzw. negative Werte konnte für den Temperaturbereich von $800^{\circ} \mathrm{C}-850^{\circ} \mathrm{C}$ nicht bestätigt werden. Eine Facettierung, die an diesen Korngrenzen häufig beobachtet wird, konnte entlang der $<110>$-Richtung durch HR-TEM Messungen ausgeschlossen werden. Die Lunkerbildung und deren Facettierung wurden auf eine gegenüber der Korngrenzsegregation bevorzugte Segregation an freien Oberflächen zurückgeführt, die bereits während der Dotierung der Bikristalle eingesetzt hat. 
In dieser Arbeit wurden die Gefüge verschieden hergestellter Ag-Ni- und $\mathrm{Cu}-\mathrm{Bi}$ - Proben charakterisiert und deren Korngrenzsegregation bei thermischer Behandlung untersucht. Dabei konnten erstmalig kompaktierte Proben und Proben mit einer einzelnen, wohldefinierten Korngrenze, mit Hilfe der 3d-Atomsondentomographie untersucht werden. Die Kombination verschiedenster Herangehensweisen (thermische Verdampfung, Sputter-Deposition, kontrollierte Bikristallzucht) und Analyseverfahren (SEM, TEM, FIM, TAP, ICP-OES, XRD) war notwendig, um die Korngrenzsegregation in den experimentell schwierig erfassbaren Legierungssystemen auf der Subnanometerskala verfolgen zu können. FIM und TAP bieten als einzige Methoden die Möglichkeit der Analyse mit gleichzeitig atomarer und chemischer Auflösung und sind somit bestens geeignet zur Untersuchung der Korngrenzsegregation. Die mangelnde Ortsselektion der Methoden konnte durch die in dieser Arbeit entwickelte und erfolgreich angewandte „Lift-Out“-Präparation mittels FIB eliminiert werden. Somit wurde eine seit der Erfindung des ersten Feld-IonenMikroskops von Erwin Müller im Jahre 1951 bestehende Schwäche der Methode überwunden. Dies eröffnet nun erstmalig die Möglichkeit ortsselektiver chemischer Analysen mittels FIM/TAP an einer einzelnen Korngrenze oder gar jeglichem nanoBereich von allgemeinem Interesse. Die Ausweitung dieser Präparationsmethode auf andere materialphysikalische Probleme ist bereits angelaufen und wird Ergebnisse liefern können, die vor kurzer Zeit noch nicht denkbar gewesen wären. 



\section{Literaturverzeichnis}

[Alchagirov04] B. B. Alchagirov, A. G. Mozgovoi, T. M. Shamparov, High Temperature 42 (2004), 493

[AlKassab95] T. Al-Kassab, M. P. Macht, H. Wollenberger, Appl. Surf. Sci 87/88 (1995), 329-336

[Ahrens95] T. J. Ahrens (Ed.), ,Mineral Physics and Crystallography - A Handbook of Physical Constants “ (1995)

[Alber99] U. Alber, H. Müllejans, M. Rühle, Acta. Mater. 47 (1999), 4047

[BABCOCK87] S. E. Babcock, R.W. Baluffi, Phil. Mag. A 55 (1987), 643

[Barbour60] J. P. Barbour, F. M. Charbonnier, W. W. Dolan, W. P. Dyke, E. E. Martin, J. K. Trolan, Phys. Rev. 117 (1960), 1452

[Borchers96] J. A. Borchers, P.M. Gehring, R. W. Erwin, C. F. Majkrzak, J. Appl. Phys. 79 (1996), 4762

[Bronstein95] I. N. Bronstein, K. A. Semendjajew, G. Musiol, H. Mühlig, Taschenbuch der Mathematik, Thun, Frankfurt am Main, Harry Deutsch (1995)

[CARINE98] Software „CaRIne Crystallography 3.1“, Divergent S. A. (1998)

[Chang97A] L.S. Chang, B.B. Straumal, E. Rabkin, W. Gust, F. Sommer, J. Phase Equilibria 18 (1997), 128

[CHANG99B] $\quad$ L. S. Chang, E. Rabkin, B. B. Straumal, B. Baretzky, W. Gust, Acta Mater. 47 (1999), 4041

[ChAng99C] L. S. Chang, E. Rabkin, S. Hofmann, W. Gust, Acta Mater. 47 (1999), 2951

[Divinski04] D. Divinski, M. Lohmann, C. Herzig, Acta Mat. 52 (2004), 3973

[Duscher04] G. Duscher, M.F. Chisholm, U. Alber, M. Rühle, Nature Materials 3 (2004), 621

[EICKEMEYer05] J. Eickemeyer, D. Selbmann, H. Wendrock, A. Güth, B. Holzapfel, Supercond. Sci. Technol. 18 (2005), 770

[FAupel02] J. Faupel, H.-U. Krebs, A. Käufler, Y. Luo, K. Samwer; S. Vitta, J. Appl. Phys, 92 (2002), 1171

[FINNIS85] M. W. Finnis, J. E. Sinclair, Phil. Mag. A 50 (1984), 45 
[Fowler39] R. H. Fowler, E. A Guggenheim, „Statistical Thermodynamics“, Cambridge, Cambridge University Press (1939)

[FraCKIEwicz85] A. Frackiewicz, M. Biscondi, J. Phys. 46 (1985), C4-497

[Giannuzzi99] L. A. Giannuzzi, F. A. Stevie, Micron 30 (1999), 197

[Gottstein01] G. Gottstein, „Physikalische Grundlagen der Materialkunde“, Berlin, Heidelberg, New York, Springer-Verlag (2001)

[Grovenor85] C. R. M. Grovenor, A. Cerezo, J. A. Liddle, G. D. W. Smith, In: Microscopy of Semiconducting Materials, Eds. A. G. Cullis, J. Holt, Inst. Phys. Conf. Ser. 76 (1985), 423

[HAASEN94] P. Haasen, „Physikalische Metallkunde“, Berlin, Heidelberg, New York, Springer-Verlag (1994)

[HAMPE74] W. Hampe, Z. Berg, Hütten- und Salinenwesen (1874), 23, 93

[Hardouin00] O. Hardouin Duparc, S. Poulat, A. Larere, J. Thibault, L. Priester, Phil. Mag. A 80 (2000), 853

[Heumann92] Th. Heumann, Diffusion in Metallen, Berlin, Heidelberg, New York, Springer-Verlag (1992)

[HIRTH68] J. P. Hirth, J. Lothe, ,Theory of Dislocations “, New York, McGrawHill (1968)

[HöFlER93] H.J. Höfler, R.S. Averback, H. Hahn, H. Gleiter, J. Appl. Phys. 74 (1993), 3832

[Hondros74] E. D. Hondros, D. McLean, Phil. Mag. 29 (1974), 771

[Hondros96] E. D. Hondros, M. P. Seah, S. Hofmann, P. Lejcek in: „Physical metallurgy vol. 2”, editors R.W. Cahn, P. Haasen, Amsterdam, North Holland (1996), 1201

[Joshi71] A. Joshi, D.F. Stein, J. Inst. Met. 99 (1971), 178

[KitTel02] C. Kittel, „Einführung in die Festkörperphysik“, München, Wien, Oldenbourg (2002)

[KonRaD96] H. Konrad, T. Haubold, R. Birringer, H. Gleiter, Nanostruc. Mater. 7 (1996), 605-610

[KRENGEL00] U. Krengel, ,Einführung in die Wahrscheinlichkeitstheorie und Statistik“, Braunschweig / Wiesbaden, Vieweg (2000)

[LAndolt90] H. Bakker, H.P. Bonzel, C.M. Bruff, M.A. Dayananda, W. Gust, J. Horváth, I. Kaur, G.V. Kidson, A.D. LeClaire, H. Mehrer, G.E. 
Murch, G. Neumann, N. Stolica, N.A. Stolwijk, „Diffusion in Solid Metals and Alloys “ III/26, editor H. Mehrer, Springer-Verlag (1990)

[LANG02] C. Lang, Dissertation, Georg-August-Univ. Göttingen (2002)

[LEJCEK95] P. Lejcek, S.Hofmann, Crit. Rev. Solid State Mater. Sci. 20 (1995), 1

[Lezzar04] B. Lezzar, O. Khalfallah, A. Larere; V. Paidar, O. Hardouin Duparc, Acta. Mater. 52 (2004), 2809

[Li90] Z. Li, Q. Li, Y. Qin, H. Shen, Phil. Mag, A 62 (1990), 125

[LóPez03] G. A. López, W. Gust, E. J. Mittemeijer, Scripta Mater. 49 (2003), 747

[LÓPEz04] G. A. López, Dissertation, Max-Planck-Institut für Metallforschung Stuttgart (2004)

[MA05] E. Ma, Prog. Mat. Sci. 50 (2005), 413

[MAKsimova89] E.L. Maksimova, E.I. Rabkin, L.S. Shvindlerman, B.B. Straumal, Acta. Metall. 37 (1989), 1995

[Massalski90] T. B. Massalski, Binary Alloy Phase Diagrams, $2^{\text {nd }}$ Ed., ASM International, Materials Park (1990)

[MCLEAN57] D. Mclean, ,, Grain boundaries in metals”, Oxford, Clarendon (1957)

[Miller04] M. K. Miller, K. F. Russel, G. B. Thompson, Ultramicroscopy 102 (2004), 287

[Miller96] M. K. Miller, A. Cerezo, M. G. Hetherington, G. D. W. Smith, „Atom Probe Field Ion Microscopy”, Oxford, Clarendon Press (1966)

[MÜLler69] E. W. Müller, T. T. T. Tsong „Field Ion Microscopy, Principles and Applications“, New York, American Elsevier Publishing Company (1969)

[Nowak04] C. Nowak, Diplomarbeit, Georg- August- Univ. Göttingen (2004)

[PARker94] M. A. Parker, T. L. Hylton, K. R. Coffey, J. K. Howard, J. Appl. Phys. 75 (1994), 6382

[QIN95] X. Y. Qin, X. J. Wu, L. D. Zhang, Nanostruc. Mater. 5 (1995), 101110

[OverwiJk93] M. H. F. Overwijk, F. C. van der Heuvel, C. W. T. Bulle-Lieuwma, J. Vac. Sci. Technol. B11 (1995), 2021

[Russel85] J. D. Russel, A.T. Winter, Scripta Metall. 19 (1985), 575 
[SCHLEIwIES01] J. Schleiwies, Disseration, Georg-August-Univ. Göttingen (2001)

[SchÖlham99] J. Schölhammer, L.S. Chang, E. Rabkin, B. Baretzky, W. Gust, E.J. Mittemeijer, Z. Metallk. 90 (1999), 9

[Schölham01] J. Schölhammer, B. Baretzky, W. Gust, E.J. Mittemeijer, Interface Science 9 (2001), 43

[SChweINFEST04] R. Schweinfest, A.T. Paxton, M.W. Finnis, Nature 432 (2004), 1008

[SchweItz01] K. O. Schweitz, J. Bøttiger, A. L. Greer, P. J. Thomas, D. T. Foord, Phil. Mag. A 81 (2001), 1

[Sigle02] S. Sigle, L.S. Chang, W. Gust, Phil. Mag. A 82 (2002), 1595

[SIMION] Softwarepaket SIMION 3D 7.0, Scientific Instruments Services, www.sisweb.com

[Smith77] D.A. Smith, V. Vitek, R.C. Pond, Acta Mater. 25 (1977), 475

[SRIM03] Freeware-Algorithmus SRIM, www.srim.org (2003)

[Tyson77] W. R. Tyson, W. A. Miller, Surf. Sci. 62 (1977), 267

[Voce47] E. Voce, A.P.C. Hallowes, J. Inst. Metals (1947), 74, 323

[Vurpillot00] F. Vurpillot, A. Bostel, D. Blavette, Appl. Phys. Lett. 76 (2000), 3127

[WAGNER82] R. Wagner, „Field-Ion-Microscopy in Materials Science”, In: Crystals, Vol. 6, ed. H. C. Freyhardt, Berlin, Springer (1982)

[Wan99] J. Wan, Y. L. Fan, D. W. Gong, S. G. Chen, X. Q. Fan, Modelling Simul. Mater. Sci. Eng. 7 (1999), 189

[Wolde01] D. Wolde-Giorgis, Diplomarbeit, Georg-August-Univ. Göttingen (2001)

[Wolde03] D. Wolde-Giorgis, T. Al-Kassab and R. Kirchheim, Mat. Sci. Eng. A 353, Issues 1-2, (2003), 152-157

[Wolf90] D. Wolf, Acta Metall. Mater. 38 (1990), 781

[Zhang95] G.H. Li, D. Zhang, Scripta Metall. Mater. 32 (1995), 1335 


\section{Appendix: Simulationen zur Sputter-Deposition}

Es wurden Monte-Carlo-Simulationen mit dem Programm SRIM-2003 [SRIM03] durchgeführt. Der als Freeware erhältliche Algorithmus simuliert die Wechselwirkung eines auf einem amorphen Festkörper auftreffenden Atoms bestimmter Energie. Die Wahl des Stoßwinkels ist dabei zufällig. Sobald ein Target-Atom seinen Platz verlässt, wird die auf es übertragene kinetische Energie um den Wert der Bindungsenergie $E_{l}$ (Lattice Binding Energy) reduziert. Das stoßende Teilchen, sowie das delokalisierte Target-Atom, können mit ihrer Energie weitere Stoßprozesse, bis hin zu ganzen Stoßkaskaden, initiieren. Sobald die Energie eines Teilchens unter einen festgesetzten Schwellenwert $E_{d}$ (Displacement Energy) gefallen ist, finden keine weiteren Stoßprozesse mehr statt. Wenn das Ende einer gekrümmten Stoßkaskade die Targetoberfläche erreicht, können einzelne oder mehrere Teilchen aus der Oberfläche austreten. Sofern die Bindungsenergie $E_{s}$ (Surface Binding Energy) überwunden wird, verlassen die Teilchen die Oberfläche mit der verbleibenden kinetischen Energie $E_{k i n}$.

Mit dem Programm SRIM-2003 kann der mittlere Wert von $E_{k i n}$ in Abhängigkeit des Einschusswinkels für beliebige Materialien bestimmt werden. Zusätzlich erhält man einen statistischen Wert über die Anzahl der austretenden Target-Atome pro auftreffendem Teilchen.

\begin{tabular}{|l|l|l|}
\hline & $\mathrm{Cu}$ & $\mathrm{Bi}$ \\
\hline Masse $[\mathrm{amu}]$ & 63,54 & 208,9 \\
\hline Dichte $\left[\mathrm{g} / \mathrm{cm}^{3}\right]$ & 8,92 & 9,8 \\
\hline$E_{l}[\mathrm{eV}]$ & 3 & 3 \\
\hline$E_{s}[\mathrm{eV}]$ & 25 & 25 \\
\hline$E_{d}[\mathrm{eV}]$ & 3,52 & 2,17 \\
\hline
\end{tabular}

Die über jeweils 100.000 Sputterprozesse gemittelten Sputteryields für $\mathrm{Cu}$ und $\mathrm{Bi}$ finden sich in Tabelle 7.1. Aufgrund der Geometrie des UHV-Rezipienten findet der 
primäre Sputterprozess unter einem Winkel von $45^{\circ}$ statt. Alle deponierten Schichten wurden mit einem $600 \mathrm{eV}$ Ar-Ionenstrahl hergestellt.

\begin{tabular}{|c|c|c|c|}
\hline Primär-Sputterprozess & Winkel & Sputter yield & {$[\mathrm{eV} /$ Atom $]$} \\
\hline $\mathrm{Cu}$ & $45^{\circ}$ & 3,58 & 19,15 \\
\hline $\mathrm{Bi}$ & $45^{\circ}$ & 2,39 & 17,68 \\
\hline Sekundär-Prozess & \multicolumn{3}{|l}{} \\
\hline $\mathrm{Cu}, \mathrm{Bi} \rightarrow \mathrm{Cu}, \mathrm{Bi}$ & $0^{\circ}$ & 0,00 & 0,00 \\
\hline $\mathrm{Cu} 70 \mathrm{eV} \rightarrow \mathrm{Bi}$ & $0^{\circ}$ & 0,43 & 10,58 \\
\hline $\mathrm{Cu} 70 \mathrm{eV} \rightarrow \mathrm{Cu}$ & $0^{\circ}$ & 0,28 & 5,86 \\
\hline $\mathrm{Bi} 70 \mathrm{eV} \rightarrow \mathrm{Cu}$ & $0^{\circ}$ & 0,0087 & 1,85 \\
\hline $\mathrm{Bi} 70 \mathrm{eV} \rightarrow \mathrm{Bi}$ & $0^{\circ}$ & 0,21 & 5,22 \\
\hline
\end{tabular}

Tab. 7.1: Jeweils über 100.000 Sputterprozesse gemittelte Sputteryields für Cu und Bi

Der primäre Sputteryield von $\mathrm{Cu}$ ist ca. $30 \%$ höher als der des Bi. Die mit ihren jeweiligen Energien ausgelösten Atome der beiden Sorten treffen dann auf die WSubstratspitze. Dabei stellt sich nun die Frage, inwieweit die sich einstellende Schichtzusammensetzung von Resputter-Effekten abhängt ${ }^{14}$. Für die mittlere Energie von $19,15 \mathrm{eV}$ pro $\mathrm{Cu}$-Atom und $17,68 \mathrm{eV}$ pro Bi-Atom verschwindet der Sputteryield. Bei dem primären Sputterprozess liegt aber keine scharfe Energieverteilung der ausgelösten Atome vor. Es handelt sich vielmehr um eine Maxwellverteilung, so dass auch wesentlich höhere Energien auftreten. Die Bi-Atome unterliegen deutlich größeren Resputtereffekten als die $\mathrm{Cu}$-Atome, wodurch die geringeren Bi-Konzentrationen im Vergleich zum 1:1 Übertrag in den Schichten zumindest qualitativ erklärt werden können.

\footnotetext{
${ }^{14}$ Der Einfallswinkel auf der Spitze kann in guter Nährung mit $0^{\circ}$ angegeben werden.
} 


\title{
Danksagung
}

\author{
Lebenslauf
}




\section{Danksagung}

An erster Stelle gilt mein großer Dank Herrn Prof. Dr. Reiner Kirchheim für die Ermöglichung einer Dissertation am Institut für Materialphysik, für seine wissenschaftliche Betreuung und seine stetige Bereitschaft, auf meine Fragen einzugehen.

Herrn PD. Dr. Tala'at Al-Kassab danke ich für die zahlreichen Anregungen und Diskussionen und die Gewährung aller nötigen Freiräume bei der Gestaltung dieser Arbeit.

Ein besonderer Dank geht an Frau Prof. Dr. Dagmar Gerthsen und Herrn Dr. Fabian Pérez-Willard für die ausgesprochen gute Zusammenarbeit auf dem Gebiet der FIBPräparation an den $\mathrm{Cu}$-Bi-Proben. Insbesondere möchte ich mich für ihre herzliche Einladung zum Besuch des Labors für Elektronenmikroskopie in Karlsruhe und ihre blitzschnellen Email-Antworten bedanken. Auch Herrn Dr. Gabriel Alejandro López sei für die Herstellung der Bikristalle gedankt.

Außerdem danke ich allen Mitarbeitern des Instituts für Materialphysik: Carsten Nowak für die Diskussionen über physikalische und nichtphysikalische Aspekte des Institutslebens, Michael Sobol für die effiziente Zusammenarbeit bei der Programmierung neuer Auswertungssoftware. Ahmad Shariq sei für seine Hilfsbereitschaft und seine stets positive Einstellung gedankt. Constantin Ene danke ich für die kollegiale Einteilung der Messzeiten und die gemeinsamen Wartungsarbeiten an den Geräten. Alexander Heinrich, Catharina Wille und Torben Boll danke ich für die strikte Wahrung unserer Essenszeiten. Außerdem danke ich den Ehemaligen: Christian Lang, Christian Kluthe, Pyuck-Pa Choi und Jens Görlich, die alle ebenfalls zu einer harmonischen Arbeitsatmosphäre und dadurch zum Gelingen dieser Arbeit beigetragen haben.

Allen Technikern der Werkstätten, besonders Herrn Tobias Schultz („Schobi Tulz“), danke ich für die unbürokratische Hilfe bei Problemen und die schnelle Umsetzung von Konzepten.

Meinen Eltern danke ich für ihre moralische und finanzielle Unterstützung.

Nicht zuletzt möchte ich meiner Verlobten Denise von ganzem Herzen danken, die mir auch in angespannten Zeiten gezeigt hat, dass es ein Leben außerhalb des Instituts gibt. 


\section{Lebenslauf}

\section{Persönliche Daten}

Name: Daniel Wolde-Giorgis

Geburtsort: $\quad$ Mainz

Geburtsdatum: $\quad$ 27. März 1976

Nationalität: deutsch

\section{Ausbildung}

Schulbildung

1995

$07 / 98$

$08 / 00$ bis $10 / 01$

$11 / 01$

seit $01 / 02$
Zivildienst $\quad 10 / 95$ bis 10/96 Zivildienst in der Afrikanisch-Asiatischen

Allgemeine Hochschulreife

Theodor-Heuss-Gymnasium Göttingen Studienförderung, AASF e.V., Göttingen (universitätsnahe Einrichtung)

Diplom - Physik Studium an der Georg - August - Universität zu Göttingen

Vordiplomprüfung Physik

Diplomarbeit am Institut für Materialphysik über „Frühstadien der Entmischung von $\mathrm{Cu}-0,7$ At. $\% \mathrm{Ti}$ untersucht mit der tomographischen Atomsonde“.

Diplomprüfung im Fach Physik

Wissenschaftlicher Mitarbeiter am Institut für Materialphysik der Georg - August - Universität $\mathrm{zu}$ Göttingen in der Arbeitsgruppe von Prof. Dr. R. Kirchheim 